\title{
Design, Synthesis, and Evaluation of a Luminescent Cholesterol Mimic
}

Emily M. Work ${ }^{\dagger}$, Guillermo Ferraudi ${ }^{\ddagger}$, Luke Kiefer ${ }^{\dagger}$, Gang Liu ${ }^{\dagger}$, Michael Grigalunas ${ }^{\dagger}$, Atul Bhardwaj $^{\dagger}$, Rasmin Kaur ${ }^{\S}$, Janel M. Dempsey ${ }^{\dagger}$, Daniel Wüstner ${ }^{\S}$, Paul Helquist ${ }^{* \dagger}$, Olaf Wiest ${ }^{* \dagger}$

$\dagger$ Department of Chemistry and Biochemistry, University of Notre Dame, Notre Dame, Indiana 46566, United States

Radiation Research Laboratory, University of Notre Dame, Notre Dame, Indiana 46556, United States

$\S$ Department of Biochemistry and Molecular Biology, University of Southern Denmark, DK5230 Odense M, Denmark

\section{Supplementary Information}

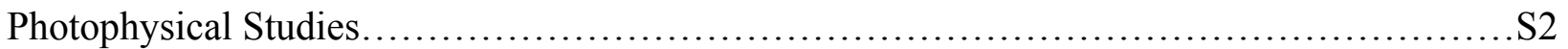

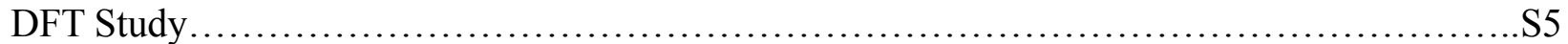

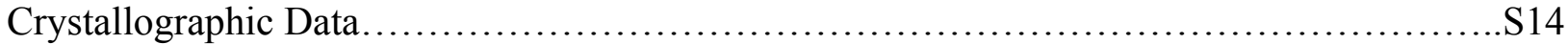

Resolution and Assignment of Absolute Configuration....................................... 17

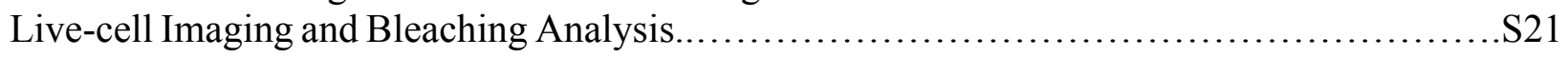

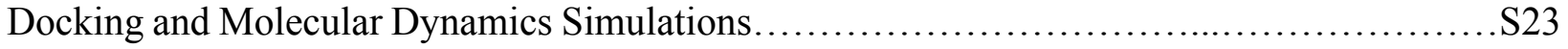

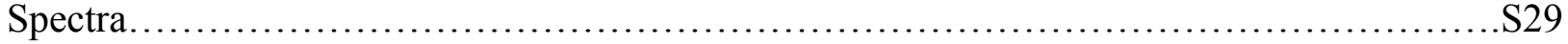




\section{Photophysical Studies}

A $1 \mathrm{mM}$ stock solution was prepared from $0.73 \mathrm{mg}$ of 1 in $2.089 \mathrm{~mL}$ of DMSO. This stock solution was diluted to the desired concentration in the desired solvent. The absorbance and emission spectra were collected for $10 \mu \mathrm{M}$ solutions of 1 in water, methanol, and 1-octanol in a 1$\mathrm{cm}$ cuvette at $22{ }^{\circ} \mathrm{C}$. Absorbance spectra were collected for a range of $240 \mathrm{~nm}-500 \mathrm{~nm}$ and emission spectra were collected with an excitation wavelength of $310 \mathrm{~nm}$, a slit width of $1 \mathrm{~nm}$, and an emission range of $350-600 \mathrm{~nm}$. The molar absorptivity was determined in methanol at 310 $\mathrm{nm}$ (Figure $\mathrm{S} 1$ ). In order to visually demonstrate the resistance to photobleaching, a $10 \mu \mathrm{M}$ solution of 1 in methanol was irradiated with $254 \mathrm{~nm}$ light from a handheld UV lamp for $30 \mathrm{~min}$. An absorbance of 0.08 was used to determine the quantum yield relative to anthracene $\left(\Phi_{\mathrm{f}}=0.27\right.$ in ethanol) ${ }^{1}$ standard.

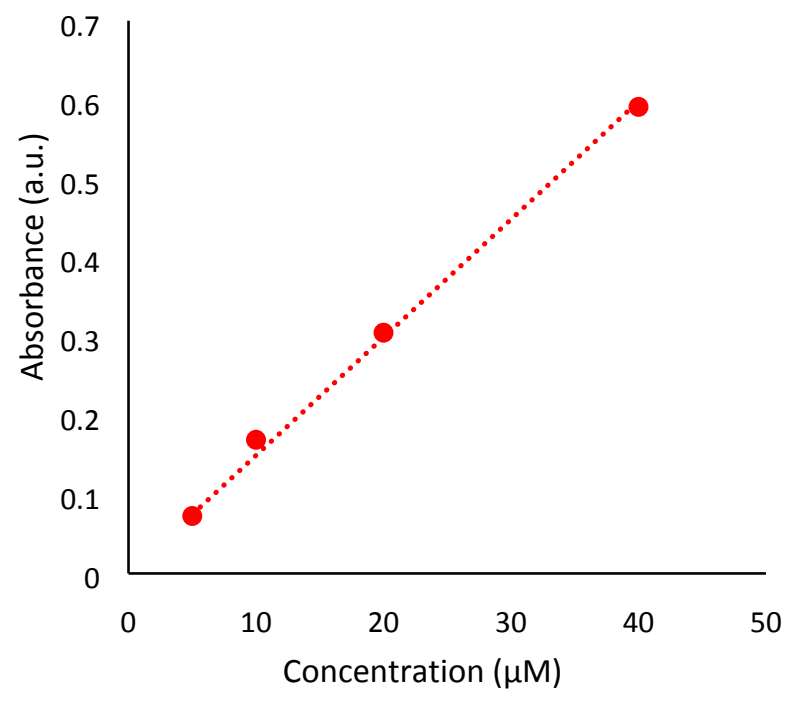

Figure S1: Absorbance at $310 \mathrm{~nm}$ vs. concentration of 1 in methanol for determination of molar absorptivity.

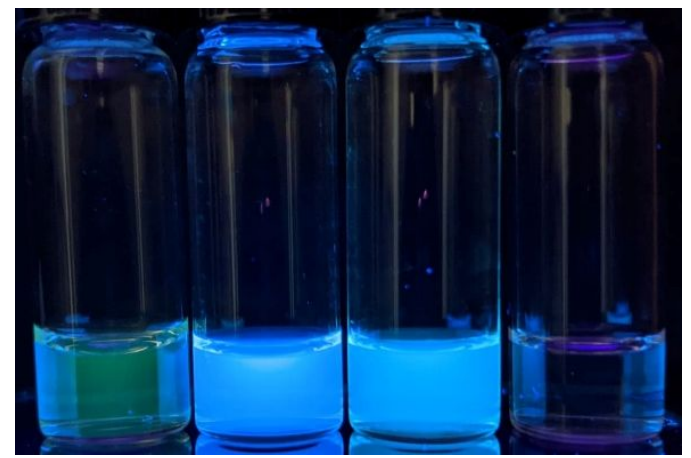

Figure S2: Irradiation with handheld UV lamp (365 nm) of $10 \mu \mathrm{M}$ solutions of (left to right) 1 in water, $\mathbf{1}$ in methanol, $\mathbf{1}$ in octanol, and DHE in methanol. 
Flash photolysis experiments were conducted in a stationary cell with irradiation at 308 $\mathrm{nm}$. The transient absorption spectrum was collected (Figure 6 in main text), and a trace was recorded at $\lambda_{\mathrm{ob}}=360 \mathrm{~nm}$ to show the decay of the spectrum, which was shown to have second order kinetics (Figure S3). No appreciable change in the baseline absorbance was observed throughout the experiments, confirming the absence of photobleaching of the sample. Irradiation of the solution at $308 \mathrm{~nm}$ produced the emission spectrum shown in Figure S4. The decay of this spectrum at $\lambda_{\mathrm{ob}}=360 \mathrm{~nm}$ approximately follows second order kinetics, with deviations due to some contribution of decay with first order kinetics.

(a)

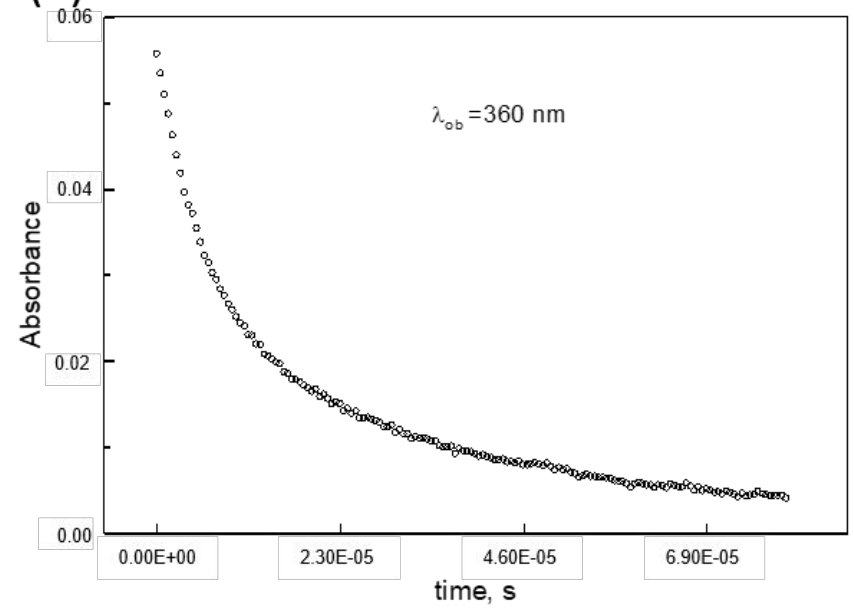

(b)

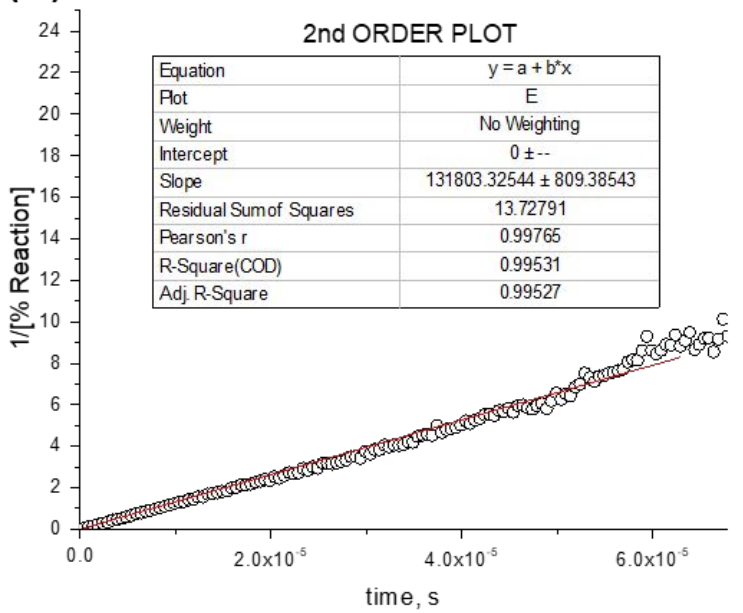

Figure S3. (a) Decay of the transient absorption of $( \pm)-1$ at $\lambda_{\mathrm{ob}}=360 \mathrm{~nm}$ in the $80 \mu$ s time domain. (b) Second order fitting of the decay. 
(a)

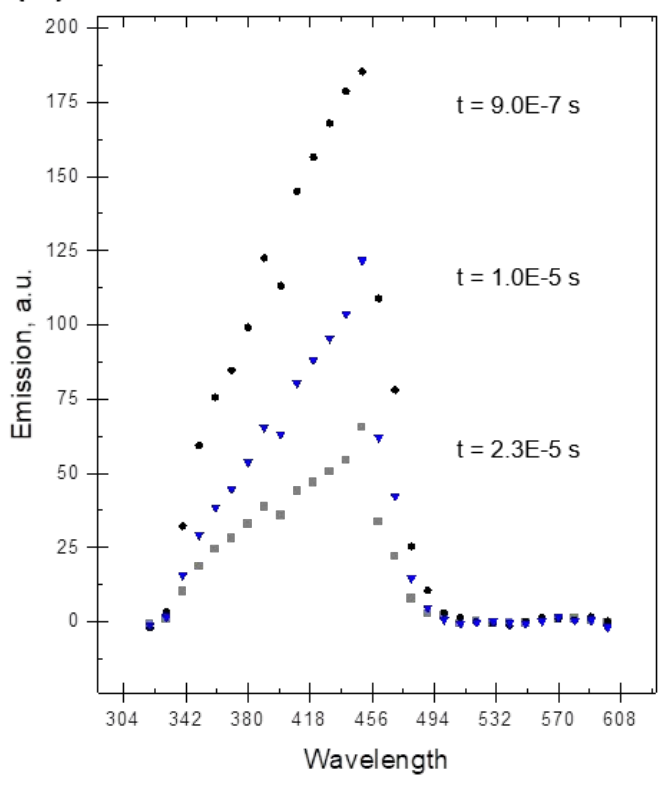

(b)

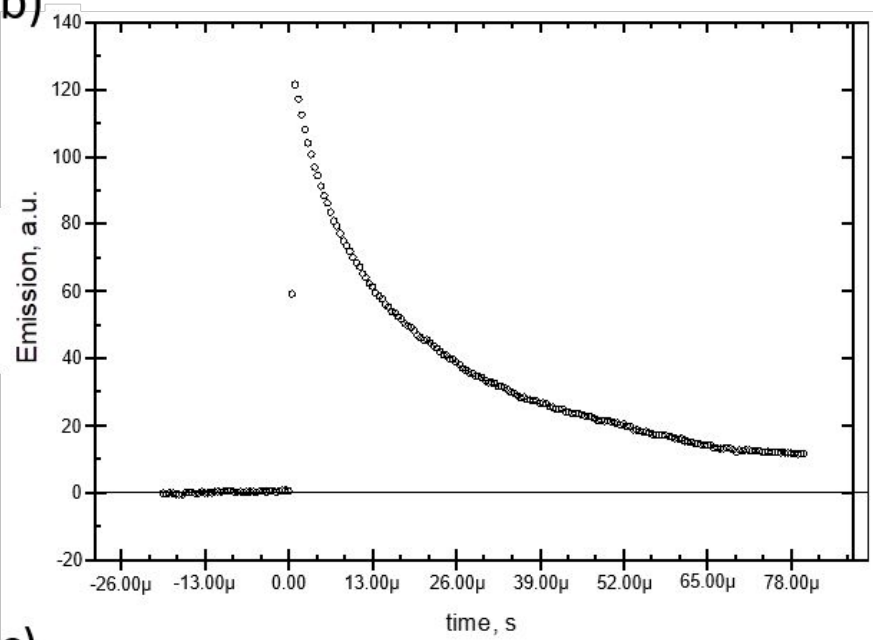

(c)

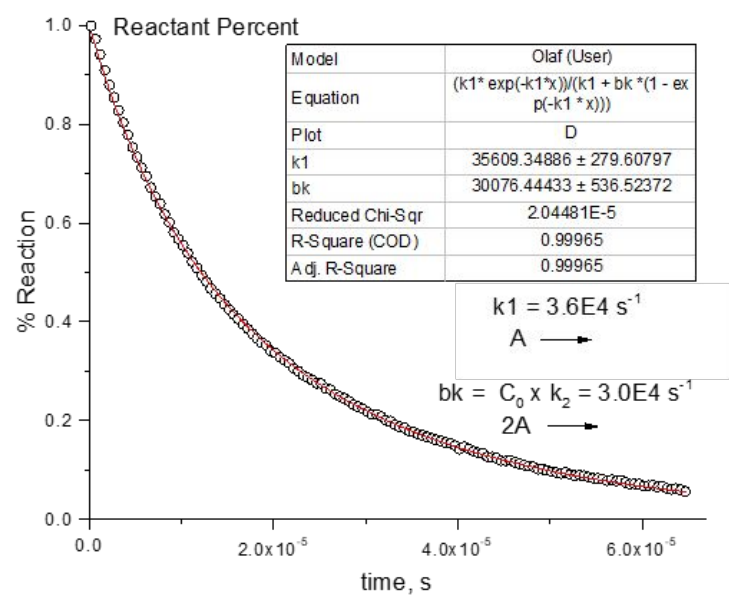

Figure S4. (a) Emission spectrum of $( \pm)-1$ in methanol upon irradiation at $308 \mathrm{~nm}$. (b) Decay of the emission at $\lambda_{\mathrm{ob}}=360 \mathrm{~nm}$ in the $80 \mu$ s time domain. (c) Combined first-second order fitting of the decay. 


\section{Computational Details}

\section{Method Selection}

Four different functionals were tested for use in calculation of spectra and excited state properties. The structure of the ground $\left(\mathrm{S}_{0}\right)$ state of 1 was optimized in the gas phase using B3LYPD3, M06-D3, CAM-B3LYP-D3, and $\omega$ B97XD functionals with the $6-311++\mathrm{G}^{* *}$ basis set using Gaussian $16 .{ }^{2}$ Frequency calculations were performed to verify that obtained stationary points were minima. Vertical excitation energies and oscillator strengths were calculated for the 15 lowest singlet excited states using time-dependent density functional theory (TDDFT) (Table S1). These results were used to simulate absorption spectra using Gaussian functions with a full width at half maximum (FWHM) of $0.3 \mathrm{eV}$ to convolute the peaks. While range-separated functionals like $\omega \mathrm{B} 97 \mathrm{XD}$ and CAM-B3LYP are commonly shown to be superior for systems with extended conjugation and charge transfer states, ${ }^{3,4}$ the B3LYP and M06 functionals were found to give better agreement with experiment for the calculated absorption spectra (Figure S5).

Table S1. Gas-phase vertical excitation energies $\Delta \mathrm{E}$ (in $\mathrm{eV}$ ) and oscillator strengths $f$ for the first fifteen singlet excited states calculated with 4 different functionals and $6-311++\mathrm{G}^{* *}$ basis set using TDDFT.

\begin{tabular}{|c|cc|cc|cc|cc|}
\hline & \multicolumn{2}{|c|}{ B3LYP-D3 } & \multicolumn{2}{c|}{ M06-D3 } & \multicolumn{2}{c|}{ (BB97XD } & \multicolumn{2}{c|}{ CAM-B3LYP-D3 } \\
\hline State & $\boldsymbol{\Delta E}$ & $\boldsymbol{f}$ & $\boldsymbol{\Delta E}$ & $\boldsymbol{f}$ & $\boldsymbol{\Delta E}$ & $\boldsymbol{f}$ & $\boldsymbol{\Delta E}$ & $\boldsymbol{f}$ \\
\hline $\mathrm{S}_{1}$ & 3.8960 & 0.0810 & 3.8965 & 0.0892 & 4.2660 & 0.0426 & 4.2601 & 0.0443 \\
\hline $\mathrm{S}_{2}$ & 4.0081 & 0.0587 & 4.0330 & 0.0450 & 4.4432 & 0.1097 & 4.4304 & 0.1126 \\
\hline $\mathrm{S}_{3}$ & 4.3202 & 0.0047 & 4.1158 & 0.0016 & 4.8560 & 0.0021 & 4.8436 & 0.0025 \\
\hline $\mathrm{S}_{4}$ & 4.7527 & 0.0067 & 4.3755 & 0.0008 & 5.3018 & 1.3277 & 5.2809 & 1.2801 \\
\hline $\mathrm{S}_{5}$ & 4.8069 & 0.8599 & 4.3924 & 0.0039 & 5.6457 & 0.2329 & 5.3281 & 0.0069 \\
\hline $\mathrm{S}_{6}$ & 4.9522 & 0.0171 & 4.6197 & 0.0043 & 5.6676 & 0.0021 & 5.6270 & 0.2548 \\
\hline $\mathrm{S}_{7}$ & 5.0277 & 0.0179 & 4.6866 & 0.0087 & 5.9293 & 0.0240 & 5.6747 & 0.0052 \\
\hline $\mathrm{S}_{8}$ & 5.1350 & 0.0698 & 4.6913 & 0.0160 & 6.0224 & 0.0102 & 5.8894 & 0.0012 \\
\hline $\mathrm{S}_{9}$ & 5.2133 & 0.3473 & 4.7838 & 0.0170 & 6.1047 & 0.0131 & 5.9104 & 0.0182 \\
\hline $\mathrm{S}_{10}$ & 5.3295 & 0.0219 & 4.8370 & 0.3169 & 6.1900 & 0.0073 & 6.0326 & 0.0049 \\
\hline $\mathrm{S}_{11}$ & 5.4000 & 0.0009 & 4.8439 & 0.5334 & 6.2241 & 0.1046 & 6.0497 & 0.0442 \\
\hline $\mathrm{S}_{12}$ & 5.4187 & 0.0022 & 4.9452 & 0.0196 & 6.2852 & 0.0963 & 6.1590 & 0.0413 \\
\hline $\mathrm{S}_{13}$ & 5.4454 & 0.0018 & 5.0174 & 0.0189 & 6.3644 & 0.0072 & 6.1982 & 0.0314 \\
\hline $\mathrm{S}_{14}$ & 5.4953 & 0.0164 & 5.0631 & 0.0112 & 6.5558 & 0.0332 & 6.2523 & 0.0316 \\
\hline $\mathrm{S}_{15}$ & 5.5705 & 0.0057 & 5.1285 & 0.1246 & 6.5916 & 0.0166 & 6.2985 & 0.0850 \\
\hline
\end{tabular}




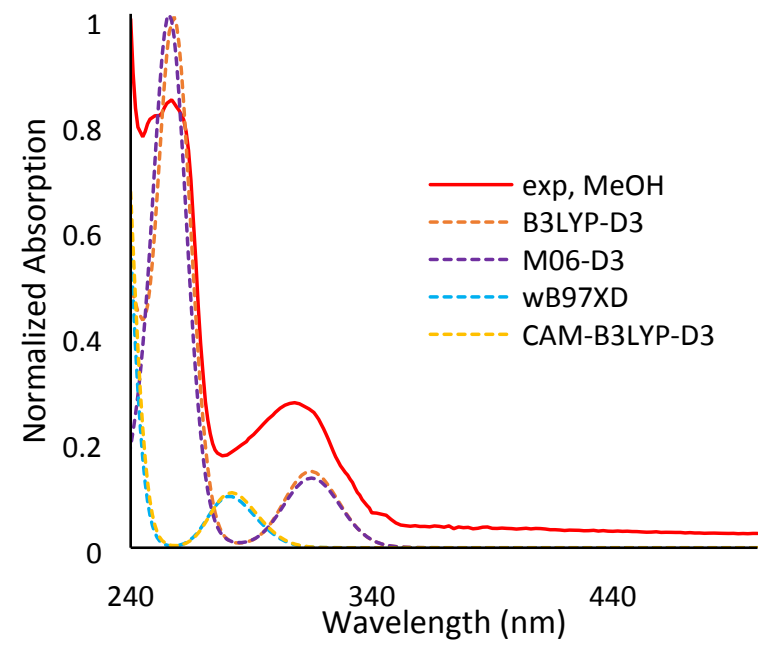

Figure S5. Comparison of experimental and calculated absorption spectra for the tested functionals. Gaussian functions with a FWHM of $0.3 \mathrm{eV}$ were used to convolute the peaks.

The lowest singlet excited $\left(\mathrm{S}_{1}\right)$ state and lowest triplet excited $\left(\mathrm{T}_{1}\right)$ state were optimized using TDDFT and the minimum character of the stationary points was verified with frequency calculations. Vertical emission energies were calculated and the corresponding wavelengths were compared with experiment (Table S2). The $\mathrm{T}_{1}$ energies are underestimated, which is a common issue with TDDFT. ${ }^{5}$ Thus the $\mathrm{T}_{1}$ state was also optimized and the vertical emission energy calculated using the $\triangle \mathrm{SCF}$ method. This gave similar $\mathrm{T}_{1}$ geometry to that found with TDDFT, but the calculated vertical emission energy showed closer agreement with experiment. Once again, B3LYP performed well, so this functional was used for further calculations in solvent.

Table S2. Gas-phase vertical emission wavelengths for the first singlet and first triplet excited states calculated using 4 different functionals with either TDDFT or $\triangle \mathrm{SCF}$ method with the 6$311++\mathrm{G}^{* *}$ basis set.

\begin{tabular}{|c|c|c|c|}
\hline & \multicolumn{3}{|c|}{$\lambda_{\mathrm{em}}(\mathrm{nm})$} \\
\hline Method & $S_{1}$ (TDDFT) & $T_{1}$ (TDDFT) & $T_{1}(\triangle S C F)$ \\
\hline B3LYP-D3 & 359.72 & 684.68 & 585.86 \\
\hline M06-D3 & 361.16 & 751.42 & 590.86 \\
\hline$\omega B 97 X D$ & 329.15 & 792.92 & 571.61 \\
\hline $\begin{array}{l}\text { CAM-B3LYP-D3 } \\
\end{array}$ & 330.61 & - & 588.09 \\
\hline
\end{tabular}




\section{Computational Results}

The structure of 1 in the $\mathrm{S}_{0}$ state was optimized using B3LYP-D3/6-311++G** and solvent effects of n-octanol were included using the polarizable continuum model (PCM). Vertical excitation energies and oscillator strengths were calculated for the 15 lowest singlet and triplet excited states using TDDFT (Table S3). These results were used to simulate absorption spectra using Gaussian functions with a FWHM of $0.3 \mathrm{eV}$ to convolute the peaks. The first excited state corresponds to the transition from the highest occupied molecular orbital (HOMO) to the lowest unoccupied molecular orbital (LUMO) (Figure S6).

Table S3. Vertical excitation energies $\Delta \mathrm{E}$ (in $\mathrm{eV}$ ) and oscillator strengths $f$ for the first fifteen singlet and triplet excited states in 1-octanol calculated at the TDDFT/PCM/B3LYP-D3/6$311++\mathrm{G}^{* *}$ level of theory at the equilibrium geometry of the $\mathrm{S}_{0}$ and $\mathrm{S}_{1}$ states.

\begin{tabular}{|c|c|c|c|c|c|c|c|}
\hline \multirow[b]{2}{*}{ No } & \multicolumn{3}{|c|}{$S_{0}$ Geometry } & \multirow[b]{2}{*}{ No } & \multicolumn{3}{|c|}{$S_{1}$ Geometry } \\
\hline & State & $\Delta \mathbf{E}$ & $f$ & & State & $\Delta \mathbf{E}$ & $f$ \\
\hline 1 & $\mathrm{~T}_{1}$ & 2.5590 & - & 1 & $\mathrm{~T}_{1}$ & 1.9808 & - \\
\hline 2 & $\mathrm{~T}_{2}$ & 3.3691 & - & 2 & $\mathrm{~T}_{2}$ & 3.0800 & - \\
\hline 3 & $\mathrm{~T}_{3}$ & 3.6539 & - & 3 & $\mathrm{~S}_{1}$ & 3.3120 & 0.3815 \\
\hline 4 & $\mathrm{~T}_{4}$ & 3.8703 & - & 4 & $\mathrm{~T}_{3}$ & 3.4680 & - \\
\hline 5 & $\mathrm{~S}_{1}$ & 3.8868 & 0.1545 & 5 & $\mathrm{~T}_{4}$ & 3.6703 & - \\
\hline 6 & $\mathrm{~T}_{5}$ & 3.9527 & - & 6 & $\mathrm{~T}_{5}$ & 3.7004 & - \\
\hline 7 & $\mathrm{~S}_{2}$ & 3.9825 & 0.0856 & 7 & $\mathrm{~S}_{2}$ & 3.7315 & 0.0563 \\
\hline 8 & $\mathrm{~T}_{6}$ & 3.9914 & - & 8 & $\mathrm{~T}_{6}$ & 3.9024 & - \\
\hline 9 & $\mathrm{~S}_{3}$ & 4.3998 & 0.0082 & 9 & $\mathrm{~S}_{3}$ & 4.1219 & 0.0076 \\
\hline 10 & $\mathrm{~T}_{7}$ & 4.4217 & - & 10 & $\mathrm{~T}_{7}$ & 4.2452 & - \\
\hline 11 & $\mathrm{~S}_{4}$ & 4.6934 & 1.1263 & 11 & $\mathrm{~S}_{4}$ & 4.3167 & 1.3908 \\
\hline 12 & $\mathrm{~T}_{8}$ & 4.9748 & - & 12 & $\mathrm{~S}_{5}$ & 4.7483 & 0.0658 \\
\hline 13 & $\mathrm{~S}_{5}$ & 5.0130 & 0.1000 & 13 & $\mathrm{~T}_{8}$ & 4.7577 & - \\
\hline 14 & $\mathrm{~T}_{9}$ & 5.0505 & - & 14 & $\mathrm{~S}_{6}$ & 4.8232 & 0.0036 \\
\hline 15 & $\mathrm{~T}_{10}$ & 5.0647 & - & 15 & $\mathrm{~T}_{9}$ & 4.8245 & - \\
\hline 16 & $\mathrm{~S}_{6}$ & 5.0741 & 0.0144 & 16 & $\mathrm{~T}_{10}$ & 4.8934 & - \\
\hline 17 & $\mathrm{~S}_{7}$ & 5.0920 & 0.1263 & 17 & $\mathrm{~T}_{11}$ & 4.9357 & - \\
\hline 18 & $\mathrm{~T}_{11}$ & 5.1173 & - & 18 & $\mathrm{~S}_{7}$ & 4.9910 & 0.0326 \\
\hline 19 & $\mathrm{~S}_{8}$ & 5.1895 & 0.2092 & 19 & $\mathrm{~S}_{8}$ & 5.0431 & 0.0379 \\
\hline 20 & $\mathrm{~T}_{12}$ & 5.2737 & - & 20 & $\mathrm{~T}_{12}$ & 5.0596 & - \\
\hline 21 & $\mathrm{~T}_{13}$ & 5.3146 & - & 21 & $\mathrm{~S}_{9}$ & 5.0660 & 0.1443 \\
\hline 22 & $\mathrm{~S}_{9}$ & 5.3175 & 0.0045 & 22 & $\mathrm{~T}_{13}$ & 5.0859 & - \\
\hline 23 & $\mathrm{~S}_{10}$ & 5.4622 & 0.0024 & 23 & $\mathrm{~S}_{10}$ & 5.1677 & 0.0302 \\
\hline 24 & $\mathrm{~T}_{14}$ & 5.4680 & - & 24 & $\mathrm{~T}_{14}$ & 5.2055 & - \\
\hline 25 & $\mathrm{~S}_{11}$ & 5.4787 & 0.0003 & 25 & $\mathrm{~S}_{11}$ & 5.2099 & 0.0007 \\
\hline 26 & $\mathrm{~T}_{15}$ & 5.4844 & - & 26 & $\mathrm{~T}_{15}$ & 5.2546 & - \\
\hline 27 & $\mathrm{~S}_{12}$ & 5.5743 & 0.0033 & 27 & $\mathrm{~S}_{12}$ & 5.3178 & 0.0065 \\
\hline 28 & $\mathrm{~S}_{13}$ & 5.6188 & 0.0221 & 28 & $\mathrm{~S}_{13}$ & 5.3610 & 0.0106 \\
\hline 29 & $\mathrm{~S}_{14}$ & 5.6996 & 0.0205 & 29 & $\mathrm{~S}_{14}$ & 5.4228 & 0.0187 \\
\hline 30 & $\mathrm{~S}_{15}$ & 5.7293 & 0.0240 & 30 & $\mathrm{~S}_{15}$ & 5.4936 & 0.0360 \\
\hline
\end{tabular}




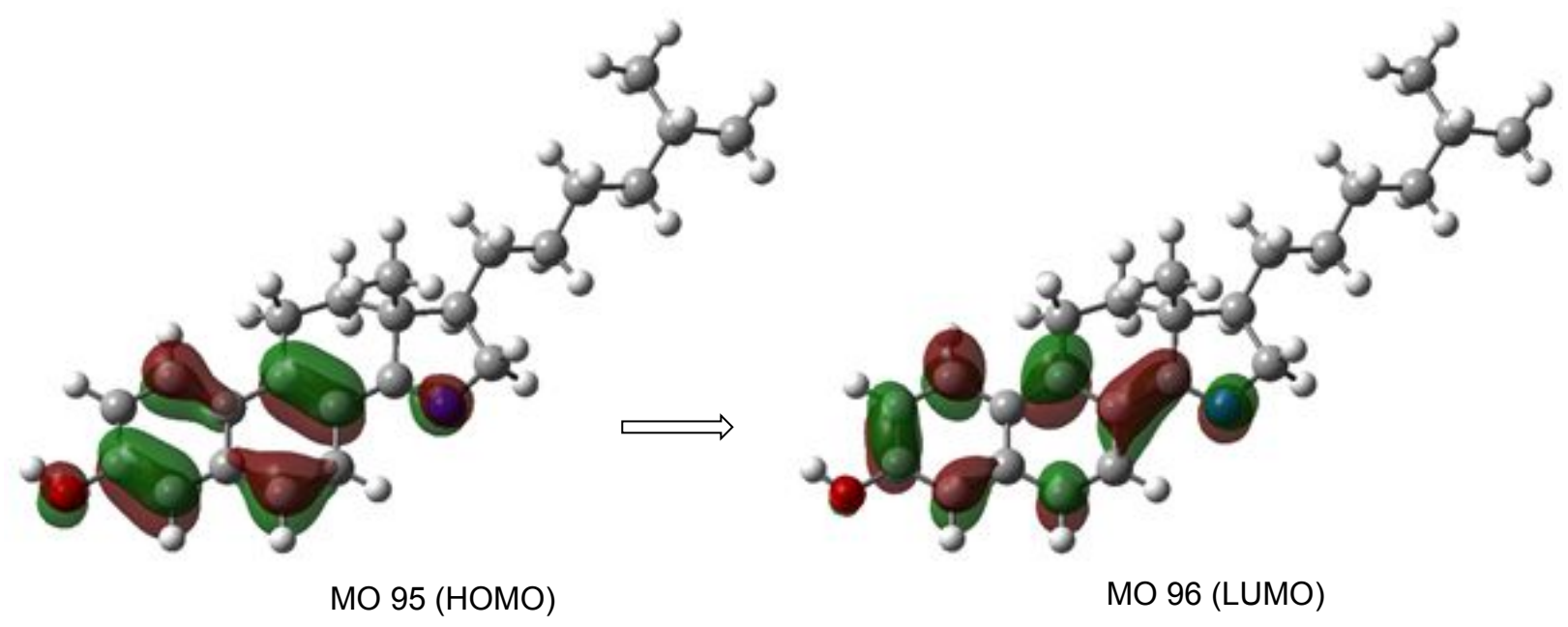

Figure S6. The highest occupied molecular orbital (HOMO) and lowest unoccupied molecular orbital (LUMO) of $\mathbf{1}$ at the equilibrium geometry of the $\mathrm{S}_{0}$ state.

The structures of the $\mathrm{S}_{1}$ and $\mathrm{T}_{1}$ states were optimized in n-octanol using PCM/TDDFT/B3LYP-D3/6-311++G** and PCM/ $\triangle$ SCF/B3LYP-D3/6-311++G**, respectively. Vertical emission energies were calculated and compared to experiment.

Comparisons of Hirshfeld charges and geometries of $\mathrm{S}_{0}, \mathrm{~S}_{1}$, and $\mathrm{T}_{1}$ were made to explain the large Stokes shift observed for emission from $T_{1}$. 
DFT Optimized Cartesian Coordinates, Energies, Hirshfeld Charges

$\mathrm{S}_{0}\left(\mathrm{PCM} / \mathrm{B} 3 \mathrm{LYP}-\mathrm{D} 3 / 6-311++\mathrm{G}^{* *}\right)$

$E=-1063.57979545$ Hartree

No imaginary frequencies

\begin{tabular}{|c|c|c|c|c|}
\hline Iement & $x$ & Y & Z & Charge \\
\hline C & 4.754501 & -1.705825 & -0.174075 & -0.034572 \\
\hline C & 6.125727 & -1.811060 & -0.164119 & -0.057552 \\
\hline C & 6.926925 & -0.647948 & -0.102729 & 0.068110 \\
\hline C & 6.337098 & 0.595441 & -0.048550 & -0.066488 \\
\hline C & 4.929219 & 0.724097 & -0.052312 & -0.001869 \\
\hline C & 4.309318 & 2.003985 & 0.018317 & -0.048820 \\
\hline C & 2.948378 & 2.113145 & 0.034337 & -0.043154 \\
\hline C & 2.114835 & 0.959367 & -0.025434 & -0.023843 \\
\hline C & 2.674691 & -0.311912 & -0.121652 & 0.012739 \\
\hline C & 4.102337 & -0.444464 & -0.121083 & -0.017174 \\
\hline C & 1.806525 & -1.554446 & -0.209134 & -0.044484 \\
\hline C & 0.335879 & -1.275393 & -0.550824 & -0.045822 \\
\hline C & -0.226077 & -0.103850 & 0.260832 & 0.010308 \\
\hline C & 0.654079 & 1.115389 & 0.026812 & 0.063710 \\
\hline$N$ & 0.034518 & 2.231358 & -0.100258 & -0.197735 \\
\hline C & -1.414270 & 1.964718 & -0.015543 & -0.020731 \\
\hline C & -1.585742 & 0.445993 & -0.249680 & -0.018157 \\
\hline C & -0.241268 & -0.423171 & 1.772050 & -0.084611 \\
\hline $\mathrm{O}$ & 8.293403 & -0.717503 & -0.093771 & -0.196902 \\
\hline C & -2.851803 & -0.174644 & 0.339181 & -0.046917 \\
\hline $\mathrm{H}$ & 8.580246 & -1.637681 & -0.122819 & 0.180697 \\
\hline C & -4.138448 & 0.333296 & -0.322325 & -0.045091 \\
\hline C & -5.403628 & -0.314109 & 0.249489 & -0.046652 \\
\hline C & -6.688052 & 0.188436 & -0.418366 & -0.047346 \\
\hline C & -7.996406 & -0.345999 & 0.191367 & -0.008131 \\
\hline C & -9.207674 & 0.353431 & -0.440835 & -0.086866 \\
\hline C & -8.125648 & -1.869583 & 0.051642 & -0.087438 \\
\hline $\mathrm{H}$ & 4.170925 & -2.615644 & -0.215943 & 0.047156 \\
\hline $\mathrm{H}$ & 6.599715 & -2.786536 & -0.204600 & 0.050998 \\
\hline $\mathrm{H}$ & 6.961120 & 1.480440 & 0.001202 & 0.046766 \\
\hline $\mathrm{H}$ & 4.934805 & 2.888733 & 0.069719 & 0.045004 \\
\hline $\mathrm{H}$ & 2.470997 & 3.082906 & 0.100607 & 0.033748 \\
\hline $\mathrm{H}$ & 2.213746 & -2.231327 & -0.964478 & 0.039370 \\
\hline $\mathrm{H}$ & 1.877393 & -2.096847 & 0.741552 & 0.037439 \\
\hline $\mathrm{H}$ & 0.259472 & -1.028544 & -1.615493 & 0.02993 \\
\hline $\mathrm{H}$ & -0.260090 & -2.179175 & -0.387698 & 0.03445 \\
\hline
\end{tabular}




\begin{tabular}{|c|c|c|c|c|}
\hline $\mathrm{H}$ & -1.946871 & 2.578118 & -0.745786 & 0.031009 \\
\hline $\mathrm{H}$ & -1.776195 & 2.264869 & 0.977679 & 0.030615 \\
\hline $\mathrm{H}$ & -1.603052 & 0.286384 & -1.335896 & 0.027100 \\
\hline $\mathrm{H}$ & -0.663813 & 0.405208 & 2.345538 & 0.032437 \\
\hline $\mathrm{H}$ & -0.840792 & -1.315873 & 1.968184 & 0.033504 \\
\hline $\mathrm{H}$ & 0.767959 & -0.601844 & 2.148546 & 0.029823 \\
\hline $\mathrm{H}$ & -2.801600 & -1.265607 & 0.232073 & 0.027307 \\
\hline $\mathrm{H}$ & -2.900513 & 0.028450 & 1.414821 & 0.026410 \\
\hline $\mathrm{H}$ & -4.206658 & 1.421763 & -0.205607 & 0.026059 \\
\hline $\mathrm{H}$ & -4.089584 & 0.143787 & -1.402438 & 0.025301 \\
\hline $\mathrm{H}$ & -5.323672 & -1.401629 & 0.141675 & 0.024583 \\
\hline $\mathrm{H}$ & -5.455644 & -0.114809 & 1.327995 & 0.024586 \\
\hline $\mathrm{H}$ & -6.665968 & -0.066337 & -1.487145 & 0.023192 \\
\hline $\mathrm{H}$ & -6.702436 & 1.284614 & -0.366671 & 0.024353 \\
\hline $\mathrm{H}$ & -7.986936 & -0.102896 & 1.262969 & 0.024105 \\
\hline $\mathrm{H}$ & -10.144048 & 0.010753 & 0.009872 & 0.027093 \\
\hline $\mathrm{H}$ & -9.150393 & 1.439146 & -0.316638 & 0.026946 \\
\hline $\mathrm{H}$ & -9.257455 & 0.141729 & -1.515020 & 0.025590 \\
\hline $\mathrm{H}$ & -7.320009 & -2.399351 & 0.565619 & 0.026877 \\
\hline $\mathrm{H}$ & -9.073392 & -2.220840 & 0.470783 & 0.027250 \\
\hline $\mathrm{H}$ & -8.099080 & -2.160812 & -1.004827 & 0.025788 \\
\hline
\end{tabular}

$\mathrm{S}_{1}$ (TDDFT/PCM/B3LYP-D3/6-311++G**)

$E=-1063.44832648$ Hartree

No imaginary frequencies

\begin{tabular}{ccccc} 
Element & $X$ & $Y$ & Z & \multicolumn{1}{c}{ Charge } \\
C & 4.749401 & -1.738145 & -0.092425 & -0.041335 \\
C & 6.148520 & -1.860279 & -0.107154 & -0.078479 \\
C & 6.926353 & -0.713951 & -0.107852 & 0.086440 \\
C & 6.311217 & 0.576971 & -0.088251 & -0.035095 \\
C & 4.925520 & 0.708626 & -0.077540 & -0.005147 \\
C & 4.308100 & 2.001506 & -0.054945 & -0.029635 \\
C & 2.929430 & 2.132660 & -0.018845 & -0.036631 \\
C & 2.090795 & 1.014478 & -0.029252 & -0.015991 \\
C & 2.687743 & -0.323788 & -0.128297 & 0.000945 \\
C & 4.088195 & -0.465709 & -0.096281 & -0.020119 \\
C & 1.800318 & -1.537289 & -0.231699 & -0.048966 \\
C & 0.337223 & -1.221468 & -0.581665 & -0.047504 \\
C & -0.217211 & -0.069197 & 0.264538 & 0.008968 \\
C & 0.657347 & 1.161900 & 0.058220 & 0.041610 \\
N & 0.007384 & 2.288201 & -0.048984 & -0.218063
\end{tabular}




\begin{tabular}{|c|c|c|c|c|}
\hline C & -1.425362 & 1.994247 & 0.063195 & -0.025503 \\
\hline C & -1.583011 & 0.483084 & -0.225734 & -0.019033 \\
\hline C & -0.228595 & -0.431922 & 1.766141 & -0.086605 \\
\hline $\mathrm{O}$ & 8.279448 & -0.709522 & -0.120408 & -0.160483 \\
\hline C & -2.842116 & -0.172447 & 0.339562 & -0.047564 \\
\hline $\mathrm{H}$ & 8.622882 & -1.612933 & -0.131735 & 0.194276 \\
\hline C & -4.134490 & 0.347639 & -0.301115 & -0.045385 \\
\hline C & -5.393076 & -0.336390 & 0.241862 & -0.046828 \\
\hline C & -6.682547 & 0.184023 & -0.402241 & -0.047404 \\
\hline C & -7.985692 & -0.391785 & 0.180353 & -0.008170 \\
\hline C & -9.203605 & 0.327151 & -0.416245 & -0.086908 \\
\hline C & -8.101116 & -1.907638 & -0.034994 & -0.087471 \\
\hline $\mathrm{H}$ & 4.160405 & -2.645242 & -0.073053 & 0.039563 \\
\hline $\mathrm{H}$ & 6.613488 & -2.839886 & -0.110340 & 0.049272 \\
\hline $\mathrm{H}$ & 6.951917 & 1.450830 & -0.089230 & 0.051964 \\
\hline $\mathrm{H}$ & 4.940998 & 2.881929 & -0.037335 & 0.047430 \\
\hline $\mathrm{H}$ & 2.478695 & 3.115949 & 0.037174 & 0.037539 \\
\hline $\mathrm{H}$ & 2.203238 & -2.226305 & -0.983160 & 0.034431 \\
\hline $\mathrm{H}$ & 1.838735 & -2.096678 & 0.715919 & 0.033153 \\
\hline $\mathrm{H}$ & 0.275167 & -0.937317 & -1.637997 & 0.028251 \\
\hline $\mathrm{H}$ & -0.273438 & -2.121428 & -0.455408 & 0.032748 \\
\hline $\mathrm{H}$ & -1.995653 & 2.628965 & -0.620718 & 0.027374 \\
\hline $\mathrm{H}$ & -1.774940 & 2.237686 & 1.080171 & 0.026918 \\
\hline $\mathrm{H}$ & -1.599010 & 0.364126 & -1.317432 & 0.025612 \\
\hline $\mathrm{H}$ & -0.663202 & 0.373938 & 2.362271 & 0.030784 \\
\hline $\mathrm{H}$ & -0.813235 & -1.339851 & 1.939510 & 0.031219 \\
\hline $\mathrm{H}$ & 0.783705 & -0.603970 & 2.137701 & 0.027924 \\
\hline $\mathrm{H}$ & -2.781131 & -1.258091 & 0.190724 & 0.026514 \\
\hline H & -2.892187 & -0.011406 & 1.422276 & 0.025467 \\
\hline $\mathrm{H}$ & -4.214708 & 1.429462 & -0.139400 & 0.025601 \\
\hline $\mathrm{H}$ & -4.083221 & 0.203787 & -1.388182 & 0.024905 \\
\hline $\mathrm{H}$ & -5.303324 & -1.417264 & 0.085607 & 0.024395 \\
\hline $\mathrm{H}$ & -5.446084 & -0.185902 & 1.328233 & 0.024358 \\
\hline $\mathrm{H}$ & -6.657749 & -0.019481 & -1.481913 & 0.023109 \\
\hline $\mathrm{H}$ & -6.707884 & 1.276328 & -0.298658 & 0.024271 \\
\hline $\mathrm{H}$ & -7.977943 & -0.202154 & 1.262715 & 0.024057 \\
\hline $\mathrm{H}$ & -10.136693 & -0.046586 & 0.016256 & 0.027012 \\
\hline $\mathrm{H}$ & -9.156522 & 1.405718 & -0.237462 & 0.026919 \\
\hline $\mathrm{H}$ & -9.251494 & 0.169462 & -1.499769 & 0.025554 \\
\hline $\mathrm{H}$ & -7.290104 & -2.454859 & 0.451501 & 0.026870 \\
\hline $\mathrm{H}$ & -9.045198 & -2.288134 & 0.366549 & 0.027158 \\
\hline $\mathrm{H}$ & -8.072828 & -2.145603 & -1.104682 & 0.025758 \\
\hline
\end{tabular}


$\mathrm{T}_{1}\left(\Delta \mathrm{SCF} / \mathrm{PCM} / \mathrm{B} 3 \mathrm{LYP}-\mathrm{D} 3 / 6-311++\mathrm{G}^{* *}\right)$

$E=-1063.49806144$ Hartree

No imaginary frequencies

\begin{tabular}{|c|c|c|c|c|c|}
\hline Element & $x$ & $Y$ & Z & Charge & $\begin{array}{c}\text { Spin } \\
\text { Density }\end{array}$ \\
\hline C & 4.752697 & -1.740504 & -0.083771 & -0.042347 & 0.246845 \\
\hline C & 6.153931 & -1.846464 & -0.100575 & -0.074713 & 0.017011 \\
\hline C & 6.925875 & -0.702845 & -0.124476 & 0.070217 & 0.172248 \\
\hline C & 6.297924 & 0.582714 & -0.128388 & -0.042848 & 0.156527 \\
\hline C & 4.923698 & 0.701704 & -0.110351 & -0.005743 & 0.024683 \\
\hline C & 4.292426 & 2.004129 & -0.117498 & -0.025603 & 0.238285 \\
\hline C & 2.924493 & 2.138290 & -0.071183 & -0.040970 & -0.027982 \\
\hline C & 2.079148 & 1.017236 & -0.025338 & -0.002565 & 0.281675 \\
\hline C & 2.687949 & -0.346121 & -0.112284 & 0.002573 & 0.372587 \\
\hline C & 4.086575 & -0.479086 & -0.096344 & -0.024284 & -0.001462 \\
\hline C & 1.791690 & -1.556971 & -0.198780 & -0.048629 & 0.024259 \\
\hline C & 0.331431 & -1.230977 & -0.552649 & -0.047208 & 0.003913 \\
\hline C & -0.216375 & -0.075057 & 0.292571 & 0.009472 & 0.000296 \\
\hline C & 0.663980 & 1.153614 & 0.093962 & 0.040816 & 0.010211 \\
\hline$N$ & 0.011556 & 2.291170 & 0.023673 & -0.196814 & 0.282224 \\
\hline C & -1.421893 & 1.992385 & 0.119004 & -0.022947 & 0.016440 \\
\hline C & -1.578295 & 0.487277 & -0.196569 & -0.018752 & 0.002596 \\
\hline C & -0.234233 & -0.439303 & 1.794148 & -0.085802 & -0.000520 \\
\hline 0 & 8.288553 & -0.692277 & -0.143619 & -0.183714 & 0.067827 \\
\hline C & -2.841411 & -0.175676 & 0.350900 & -0.047114 & 0.000295 \\
\hline $\mathrm{H}$ & 8.630402 & -1.595110 & -0.142251 & 0.186093 & 0.002704 \\
\hline C & -4.129384 & 0.354902 & -0.290045 & -0.045190 & 0.000159 \\
\hline C & -5.391401 & -0.337951 & 0.233404 & -0.046703 & 0.000001 \\
\hline C & -6.676572 & 0.193201 & -0.410495 & -0.047355 & -0.000006 \\
\hline C & -7.983402 & -0.394145 & 0.151990 & -0.008146 & 0.000000 \\
\hline C & -9.197506 & 0.335133 & -0.439803 & -0.086880 & 0.000000 \\
\hline C & -8.096197 & -1.905762 & -0.092611 & -0.087441 & 0.000000 \\
\hline $\mathrm{H}$ & 4.172745 & -2.652944 & -0.052804 & 0.041053 & 0.018366 \\
\hline $\mathrm{H}$ & 6.623562 & -2.824554 & -0.090506 & 0.048399 & 0.000324 \\
\hline $\mathrm{H}$ & 6.929404 & 1.463585 & -0.152057 & 0.049644 & 0.008102 \\
\hline $\mathrm{H}$ & 4.927047 & 2.883379 & -0.135117 & 0.048424 & 0.014347 \\
\hline $\mathrm{H}$ & 2.473960 & 3.122939 & -0.041348 & 0.037728 & -0.002728 \\
\hline $\mathrm{H}$ & 2.187783 & -2.256154 & -0.943088 & 0.035912 & 0.014571 \\
\hline $\mathrm{H}$ & 1.824821 & -2.103082 & 0.755028 & 0.034115 & 0.023907 \\
\hline $\mathrm{H}$ & 0.272496 & -0.946955 & -1.609223 & 0.028595 & 0.001094 \\
\hline $\mathrm{H}$ & -0.286283 & -2.125952 & -0.426534 & 0.033142 & -0.000029 \\
\hline
\end{tabular}




$\begin{array}{rrrrrr}\text { H } & -1.984482 & 2.640706 & -0.557860 & 0.030724 & 0.008179 \\ \text { H } & -1.772579 & 2.223416 & 1.137088 & 0.031077 & 0.019339 \\ \text { H } & -1.587835 & 0.385948 & -1.290075 & 0.026521 & 0.000200 \\ \text { H } & -0.666591 & 0.367333 & 2.390791 & 0.031373 & 0.000354 \\ \text { H } & -0.823661 & -1.344470 & 1.964290 & 0.032274 & -0.000206 \\ \text { H } & 0.776025 & -0.616655 & 2.168593 & 0.029288 & 0.002670 \\ \text { H } & -2.779731 & -1.258790 & 0.185799 & 0.027115 & 0.000019 \\ \text { H } & -2.897890 & -0.031068 & 1.435599 & 0.026126 & 0.000402 \\ \text { H } & -4.210971 & 1.433986 & -0.111487 & 0.025852 & 0.000210 \\ \text { H } & -4.070820 & 0.228486 & -1.378873 & 0.025186 & 0.000052 \\ \text { H } & -5.300469 & -1.416041 & 0.059744 & 0.024560 & -0.000002 \\ \text { H } & -5.451552 & -0.205544 & 1.321736 & 0.024509 & 0.000010 \\ \text { H } & -6.643783 & 0.009353 & -1.493466 & 0.023176 & 0.000001 \\ \text { H } & -6.703561 & 1.283421 & -0.287267 & 0.024304 & 0.000002 \\ \text { H } & -7.983536 & -0.224813 & 1.237735 & 0.024088 & 0.000000 \\ \text { H } & -10.133399 & -0.047297 & -0.021204 & 0.027061 & 0.000000 \\ \text { H } & -9.152585 & 1.410169 & -0.240399 & 0.026927 & 0.000000 \\ \text { H } & -9.237422 & 0.197820 & -1.526417 & 0.025585 & 0.000000 \\ \text { H } & -7.288426 & -2.461646 & 0.389436 & 0.026887 & 0.000000 \\ \text { H } & -9.042923 & -2.294230 & 0.294802 & 0.027211 & 0.000000 \\ \text { H } & -8.059952 & -2.123547 & -1.166342 & 0.025790 & 0.000000\end{array}$




\section{Crystallographic Data}

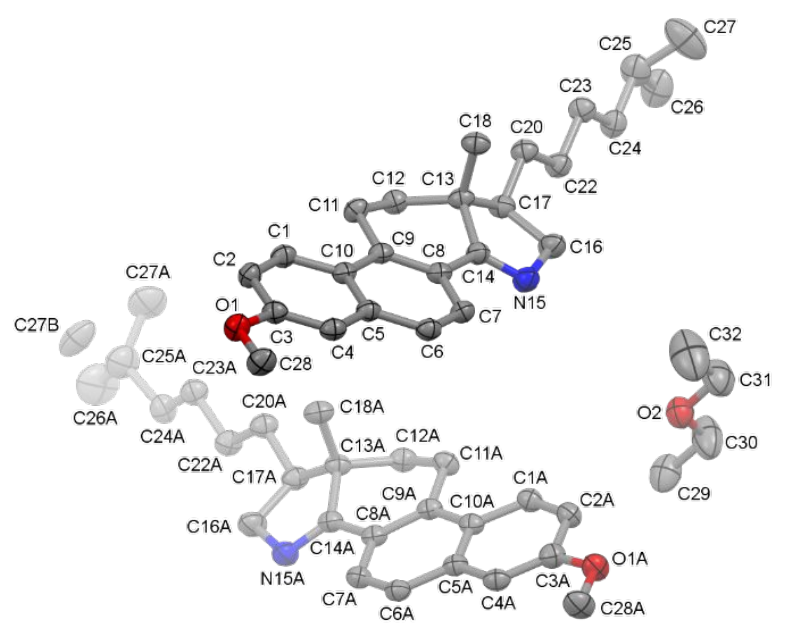

Figure S7. Crystal structure of compound 8 with thermal ellipsoids shown at 50\% probability. Hydrogen atoms are omitted for clarity.

Crystallographic details are located in the Crystallographic Information Framework (CIF) file, available from the Cambridge Crystal Data Centre (CCDC 2016041).

\section{Crystal data}

Chemical formula

$M_{\mathrm{r}}$

Crystal system, space group

Temperature $(\mathrm{K})$

$a, b, c(\AA)$

$\alpha, \beta, \gamma\left({ }^{\circ}\right)$

$V\left(\AA^{3}\right)$

$Z$

Radiation type

$\mu\left(\mathrm{mm}^{-1}\right)$

Crystal size (mm)

Data collection

Diffractometer

Absorption correction
$2\left(\mathrm{C}_{25} \mathrm{H}_{33} \mathrm{NO}\right) \cdot \mathrm{C}_{4} \mathrm{H}_{10} \mathrm{O}$

801.16

Triclinic, $P-1$

120

$9.8931(4), 16.2793(6), 16.9538(9)$

$118.201(2), 94.592(3), 96.127$ (2)

2365.91 (19)

2

$\mathrm{Cu} K \alpha$

0.52

$0.3 \times 0.14 \times 0.05$

\section{Bruker APEX-II CCD}

Numerical

TWINABS-2012/1 (Bruker,2012) was used for absorption correction.

Final HKLF 4 output contains 72837 reflections, $R_{\text {int }}=$ 0.0672 (42196 with $I>3 \sigma(I), R_{\text {int }}=0.0544$ ).

$0.596,0.753$

$8621,8621,6744$

0.067

0.612 


\section{Refinement}

$R\left[F^{2}>2 \sigma\left(F^{2}\right)\right], \mathrm{w} R\left(F^{2}\right), S$

$0.068,0.178,1.05$

No. of reflections

8621

No. of parameters

552

$\mathrm{H}$-atom treatment

H-atom parameters constrained

$\Delta \rho_{\max }, \Delta \rho_{\min }\left(\mathrm{e} \AA^{-3}\right)$

$0.24,-0.29$

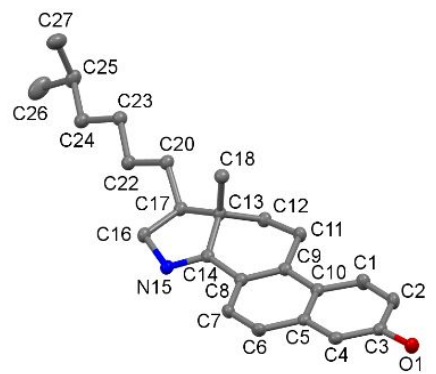

Figure S8. Crystal structure of compound $(S, S)-\mathbf{1}$ with thermal ellipsoids shown at 50\% probability. Hydrogen atoms are omitted for clarity.

Crystallographic details are located in the Crystallographic Information Framework (CIF) file, available from the Cambridge Crystal Data Centre (CCDC 2022200).

Crystal data

Chemical formula

$\mathrm{C}_{24} \mathrm{H}_{31} \mathrm{NO}$

$M_{\mathrm{r}}$

349.50

Crystal system, space group

Temperature $(\mathrm{K})$

Orthorhombic, $P 2{ }_{1} 2_{1} 2_{1}$

$a, b, c(\AA)$

$V\left(\AA^{3}\right)$

120

$Z$

$5.5822(2), 16.6432(5), 21.5617(7)$

$2003.20(11)$

Radiation type

4

$\mu\left(\mathrm{mm}^{-1}\right)$

$\mathrm{Cu} K \alpha$

0.53

Crystal size (mm)

$0.29 \times 0.04 \times 0.04$

Data collection

Diffractometer

Absorption correction

Bruker PHOTON-II

Numerical

SADABS2016/2 (Bruker,2016/2) was used for absorption correction. wR2(int) was 0.0904 before and 0.0481 after correction. The Ratio of minimum to maximum transmission is 0.9183 . The $\lambda / 2$ correction factor is Not present.

$T_{\min }, T_{\max }$

$0.918,1.000$

No. of measured, independent and 40597, 3830, 3723

observed $[I>2 \sigma(I)]$ reflections

$R_{\text {int }}$

0.033

$(\sin \theta / \lambda)_{\max }\left(\AA^{-1}\right)$

0.612 


\section{Refinement}

$R\left[F^{2}>2 \sigma\left(F^{2}\right)\right], \mathrm{w} R\left(F^{2}\right), S$

No. of reflections

No. of parameters

$\mathrm{H}$-atom treatment

$\Delta \rho_{\max }, \Delta \rho_{\min }\left(\mathrm{e} \AA^{-3}\right)$

Absolute structure

Absolute structure parameter
$0.028,0.073,1.06$

3830

239

H-atom parameters constrained

$0.13,-0.17$

Flack $\mathrm{x}$ determined using 1542 quotients [(I+)-(I) $] /[(\mathrm{I}+)+(\mathrm{I}-)]$ (Parsons, Flack and Wagner, Acta Cryst. B69 (2013) 249-259).

$0.03(6)$ 


\section{Resolution and Assignment of Absolute Configuration}

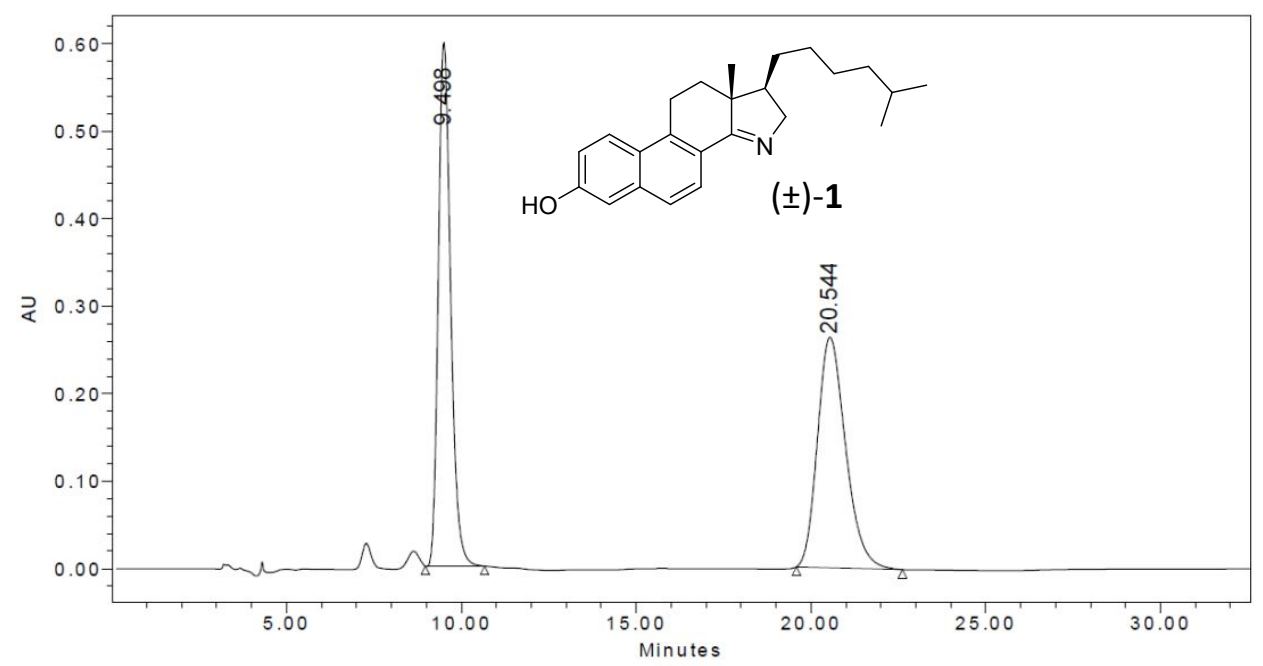

\begin{tabular}{|c|c|c|c|c|c|}
\hline & $\begin{array}{c}\mathrm{R} \mathrm{T} \\
(\mathrm{m} \text { in })\end{array}$ & $\begin{array}{c}\text { Area } \\
\left(\mu \mathrm{V}^{*} \mathrm{sec}\right)\end{array}$ & $\%$ Area & $\begin{array}{c}\text { Height } \\
(\mu \mathrm{V})\end{array}$ & $\begin{array}{c}\% \\
\text { Height }\end{array}$ \\
\hline 1 & 9.498 & 14674680 & 49.90 & 598729 & 69.46 \\
\hline 2 & 20.544 & 14735349 & 50.10 & 263302 & 30.54 \\
\hline
\end{tabular}

Stationary phase: Chiralcel OZ-H, $10 \mathrm{~mm}$ x $250 \mathrm{~mm}$ column Mobile phase: $90 / 10 / 0.1 \%$ hexane/2-propanol/diethylamine Flow rate: $4.73 \mathrm{~mL} / \mathrm{min}$ Load: $5 \mu \mathrm{L}$ injection of $10 \mathrm{mg} / \mathrm{mL}$ solution

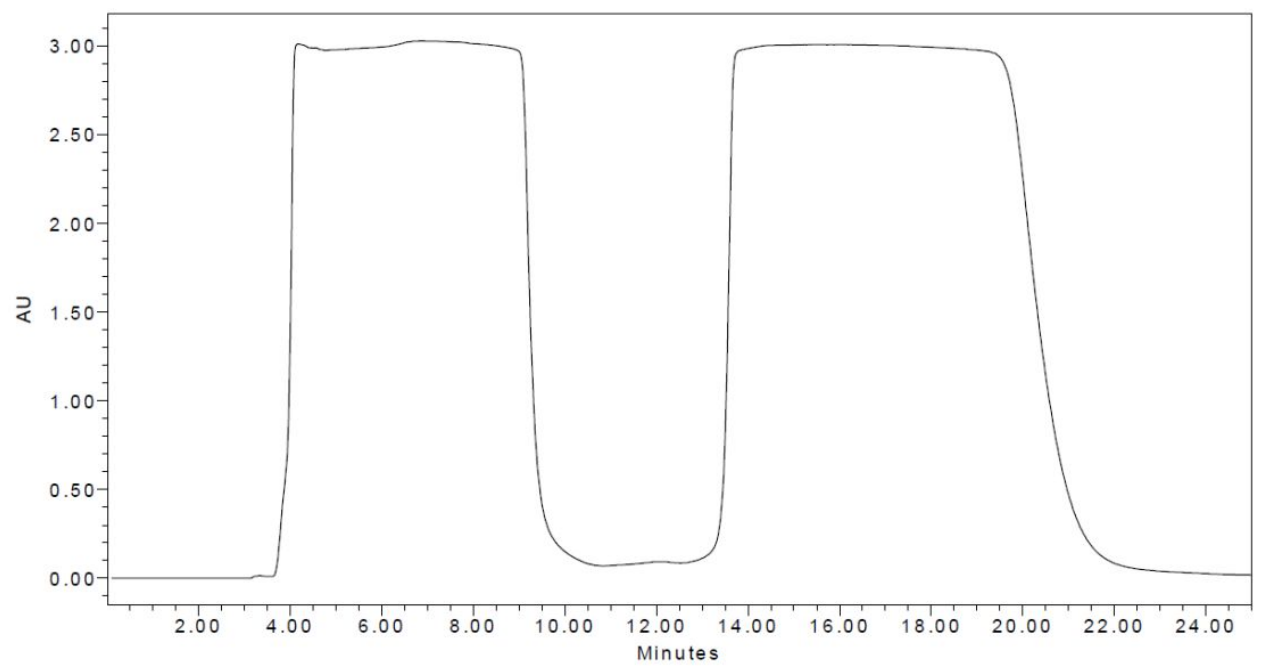

Stationary phase: Chiralcel OZ-H, $10 \mathrm{~mm}$ x $250 \mathrm{~mm}$ column Mobile phase: $90 / 10 / 0.1 \%$ hexane/2-propanol/diethylamine Flow rate: $4.73 \mathrm{~mL} / \mathrm{min}$ Load: $800 \mu \mathrm{L}$ injection of $10 \mathrm{mg} / \mathrm{mL}$ solution

Method was able to be scaled up while maintaining baseline separation. 
The absolute configuration of the later eluting enantiomer was assigned as $S, S$ by x-ray crystallography (see Figure S8 in Crystallographic Details) and this assignment was confirmed by circular dichroism (CD) spectroscopy. Using the crystal structure coordinates as a starting point, the geometry of $(S, S)-1$ in the $\mathrm{S}_{0}$ state was optimized using B3LYP-D3/6-311++ $\mathrm{G}^{* *}$ and solvent effects of 2-propanol were included using PCM. Vertical excitation energies and rotatory strengths were calculated for the 15 lowest singlet excited states using TDDFT (Table S5). These results were used to simulate the ECD spectrum using Gaussian functions $(\sigma=0.15 \mathrm{eV})$ to convolute the peaks. The simulated ECD spectrum of $(S, S)-\mathbf{1}$ showed good agreement with the experimental ECD spectrum of the later eluting enantiomer (Figure S9).

Table S4. Vertical excitation energies $\Delta \mathrm{E}$ (in $\mathrm{eV}$ ) and rotatory strengths $\mathrm{R}_{\mathrm{vel}}$ for the first fifteen singlet excited states of $(S, S)$-1 in 2-propanol calculated at the TDDFT/PCM/B3LYP-D3/6$311++\mathrm{G}^{* *}$ level of theory at the equilibrium geometry of the $\mathrm{S}_{0}$ state.

\begin{tabular}{|c|cc|}
\hline State & $\boldsymbol{\Delta E}$ & $\mathbf{R}_{\text {vel }}$ \\
\hline $\mathrm{S}_{1}$ & 3.9279 & -1.1269 \\
\hline $\mathrm{S}_{2}$ & 3.9946 & 12.5448 \\
\hline $\mathrm{S}_{3}$ & 4.4182 & -44.7729 \\
\hline $\mathrm{S}_{4}$ & 4.7037 & 55.1046 \\
\hline $\mathrm{S}_{5}$ & 5.0135 & 29.6128 \\
\hline $\mathrm{S}_{6}$ & 5.0840 & -30.7092 \\
\hline $\mathrm{S}_{7}$ & 5.1240 & -11.2034 \\
\hline $\mathrm{S}_{8}$ & 5.2221 & 7.4331 \\
\hline $\mathrm{S}_{9}$ & 5.3424 & -3.2708 \\
\hline $\mathrm{S}_{10}$ & 5.4754 & 0.3051 \\
\hline $\mathrm{S}_{11}$ & 5.5385 & 2.5359 \\
\hline $\mathrm{S}_{12}$ & 5.6155 & -3.3244 \\
\hline $\mathrm{S}_{13}$ & 5.6338 & -3.8655 \\
\hline $\mathrm{S}_{14}$ & 5.7317 & -1.5687 \\
\hline $\mathrm{S}_{15}$ & 5.7541 & -6.8654 \\
\hline
\end{tabular}

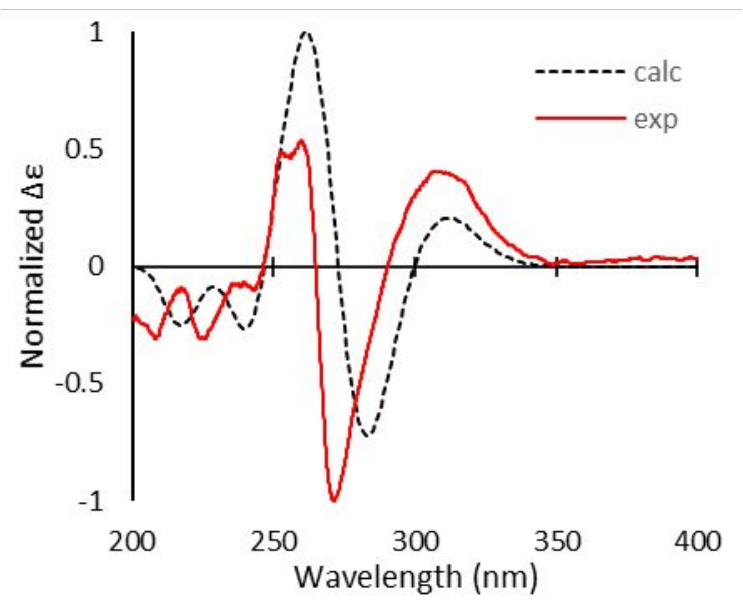

Figure S9. Comparison of experimental and calculated ECD spectra of $(S, S)-1$. Gaussian functions $(\sigma=0.15 \mathrm{eV})$ were used to convolute the peaks. 
DFT Optimized Cartesian Coordinates and Energy of (S,S)-1 in 2-propanol

$\mathrm{S}_{0}\left(\mathrm{PCM} / \mathrm{B} 3 \mathrm{LYP}-\mathrm{D} 3 / 6-311++\mathrm{G}^{* *}\right)$

$E=-1063.58141674$ Hartree

No imaginary frequencies

\begin{tabular}{|c|c|c|c|}
\hline ement & $x$ & Y & Z \\
\hline $\mathrm{O}$ & -8.296135 & -0.782364 & -0.044926 \\
\hline $\mathrm{H}$ & -8.745692 & 0.069088 & 0.017380 \\
\hline C & -4.785753 & -1.688057 & -0.169553 \\
\hline $\mathrm{H}$ & -4.213717 & -2.604261 & -0.228012 \\
\hline C & -6.155971 & -1.775693 & -0.143570 \\
\hline $\mathrm{H}$ & -6.656203 & -2.735877 & -0.185243 \\
\hline C & -6.940598 & -0.602927 & -0.060614 \\
\hline C & -6.335282 & 0.633217 & 0.001619 \\
\hline $\mathrm{H}$ & -6.936027 & 1.535184 & 0.069310 \\
\hline C & -4.923311 & 0.743777 & -0.016754 \\
\hline C & -4.286022 & 2.013579 & 0.063443 \\
\hline $\mathrm{H}$ & -4.898041 & 2.906352 & 0.135447 \\
\hline C & -2.922820 & 2.104102 & 0.063453 \\
\hline $\mathrm{H}$ & -2.432346 & 3.066786 & 0.137968 \\
\hline C & -2.106596 & 0.940784 & -0.024160 \\
\hline C & -2.686255 & -0.321765 & -0.129607 \\
\hline C & -4.114706 & -0.434514 & -0.110715 \\
\hline C & -1.837061 & -1.574940 & -0.246814 \\
\hline $\mathrm{H}$ & -2.265171 & -2.235943 & -1.004497 \\
\hline $\mathrm{H}$ & -1.901233 & -2.128911 & 0.697572 \\
\hline C & -0.368132 & -1.311679 & -0.607148 \\
\hline $\mathrm{H}$ & 0.217267 & -2.225819 & -0.465579 \\
\hline $\mathrm{H}$ & -0.304186 & -1.051023 & -1.669351 \\
\hline C & 0.222733 & -0.159835 & 0.212093 \\
\hline C & -0.642947 & 1.074959 & 0.008491 \\
\hline$N$ & -0.008762 & 2.183578 & -0.112890 \\
\hline C & 1.437459 & 1.894576 & -0.052783 \\
\hline $\mathrm{H}$ & 1.817654 & 2.174322 & 0.939554 \\
\hline $\mathrm{H}$ & 1.969004 & 2.510775 & -0.781440 \\
\hline C & 1.582320 & 0.377377 & -0.311816 \\
\hline $\mathrm{H}$ & 1.579942 & 0.233853 & -1.400388 \\
\hline C & 0.256316 & -0.500770 & 1.718334 \\
\hline $\mathrm{H}$ & 0.699827 & 0.312979 & 2.296928 \\
\hline $\mathrm{H}$ & 0.845650 & -1.404740 & 1.892090 \\
\hline $\mathrm{H}$ & -0.749464 & -0.670846 & 2.107815 \\
\hline C & 2.847865 & -0.271164 & 0.247156 \\
\hline $\mathrm{H}$ & 2.779017 & -1.359366 & 0.123802 \\
\hline
\end{tabular}




$\begin{array}{rrrr}\text { H } & 2.916750 & -0.085699 & 1.324817 \\ \mathrm{C} & 4.131395 & 0.227154 & -0.427623 \\ \mathrm{H} & 4.058196 & 0.064250 & -1.510608 \\ \mathrm{H} & 4.223489 & 1.311098 & -0.286643 \\ \mathrm{C} & 5.394144 & -0.456888 & 0.105560 \\ \mathrm{H} & 5.309721 & -1.540903 & -0.047744 \\ \mathrm{H} & 5.448665 & -0.303192 & 1.189053 \\ \mathrm{C} & 6.677069 & 0.048631 & -0.562910 \\ \mathrm{H} & 6.795400 & 1.121810 & -0.358003 \\ \mathrm{H} & 6.563529 & -0.043454 & -1.650649 \\ \mathrm{C} & 7.966014 & -0.680553 & -0.143189 \\ \mathrm{H} & 7.817732 & -1.752810 & -0.332399 \\ \mathrm{C} & 9.151086 & -0.209703 & -0.997607 \\ \mathrm{H} & 10.067766 & -0.746725 & -0.735475 \\ \mathrm{H} & 9.335508 & 0.859746 & -0.844341 \\ \mathrm{H} & 8.960624 & -0.366533 & -2.063742 \\ \mathrm{C} & 8.278169 & -0.500748 & 1.349405 \\ \mathrm{H} & 8.393774 & 0.562254 & 1.590883 \\ \mathrm{H} & 9.210601 & -1.007116 & 1.616749 \\ \mathrm{H} & 7.487673 & -0.904933 & 1.986260\end{array}$




\section{Live-cell Imaging and Bleaching Analysis of Azasterol}

\section{Cell culture and labeling}

Human skin fibroblasts were purchased from Cornell Institute (\#GM08680). Fetal bovine serum (FBS) was purchased from Gibco. All other chemicals including DMEM and human lipoprotein depleted serum (LPDS) were supplied from MERCK KGaA. The buffer medium (M1 medium) contained $150 \mathrm{mM} \mathrm{NaCl}, 5 \mathrm{mM} \mathrm{KCl}, 1 \mathrm{mM} \mathrm{CaCl}_{2}, 1 \mathrm{mM} \mathrm{MgCl} 2,5 \mathrm{mM}$ glucose and $20 \mathrm{mM}$ HEPES (pH 7.4) was prepared and kept at $4{ }^{\circ} \mathrm{C}$ until use. CTL was synthesized as described and kindly provided by Dr. Covey (Washington University, St. Louis, USA). ${ }^{6}$ Labeling solutions with either $(S, S)$-azasterol or CTL were prepared by loading the respective sterol probe onto albumin. Here, sterol was injected into a $1 \mathrm{~mL}$ solution of buffer medium with a final conc. of $0.08 \mathrm{mg} / \mathrm{mL}$ of sterol containing a 1000-fold molar excess of fatty acid-free BSA (i.e., $40 \mathrm{mg} / \mathrm{ml}$ ), while vortexing the solution. Vortexing was continued for another $5 \mathrm{~min}$. The solution was then allowed to attain equilibrium over a period of $0.5-1 \mathrm{~h}$ at room temperature. The final concentration of ethanol was under 1 percent (v/v). Cells were cultured at $37{ }^{\circ} \mathrm{C}$ in an atmosphere of $5 \% \mathrm{CO}_{2}$ until $90 \%$ confluence in complete DMEM and supplemented with antibiotics (1\% streptomycin and $1 \%$ penicillin) with 10\% FBS. The cells were placed on microscopy dishes and allowed to settle for $24 \mathrm{~h}$ before labeling. Cells were incubated for $24 \mathrm{~h}$ with the sterol/albumin complex in DMEM containing 10\% LPDS and washed extensively with M1 medium before imaging.

\section{Fluorescence microscopy and analysis of photobleaching}

Cells were imaged on a UV-sensitive Leica DMIRBE wide field microscope with a 63x 1.4 NA oil immersion objective (Leica Lasertechnik $\mathrm{GmbH}$ ) with a Lambda SC smart shutter (Sutter Instrument Company) as illumination control. Images were acquired with an Andor Ixon ${ }^{\mathrm{EM}}$ blue EMCCD camera operated at $-75^{\circ} \mathrm{C}$ under control of the Solis software provided with the camera. Azasterol and CTL were imaged in the UV using a specially designed filter cube obtained from Chroma Technology Corp. with 335-nm (20-nm bandpass) excitation filter, 365-nm dichromatic mirror and 405-nm (40-nm bandpass) emission filter. Azasterol was additionally imaged using the same type of filter cube just without any emission filter to collect photons over the whole emission range. For both conditions, image stacks were acquired with a 50\% neutral density filter and 0.4 sec acquisition time for 500 to 1000 images per stack. Image stacks were imported into ImageJ (http://rsb.info.nih.gov/ij), and bleaching was assessed on a pixel-by-pixel basis using PixBleach, an ImageJ plugin, we developed for that purpose, ${ }^{7}$ and which is available at http://bigwww.epfl.ch/algorithms/pixbleach/. To the bleaching kinetics of each pixel a stretched exponential decay function was fitted, which has the form

$$
I(x, y)=A(x, y) \cdot \exp \left[-\left(\frac{t}{\tau(x, y)}\right)^{\frac{1}{h(x, y)}}\right]+B(x, y)
$$

Here, $\mathrm{A}(\mathrm{x}, \mathrm{y})$ is the amplitude image resembling the sterol intensity, $\mathrm{h}(\mathrm{x}, \mathrm{y})$ is the map of the stretching parameters, $\tau(\mathrm{x}, \mathrm{y})$ describes the time constants and $\mathrm{B}(\mathrm{x}, \mathrm{y})$ accounts for background and 
autofluorescence. ${ }^{8}$ Histograms of stretching parameters and time constants were generated and plotted in Python as Jupyter notebook (jupyter.org).

To extract and visualize the bleaching profile, selected image stacks were analyzed by defining quadratic regions of interest of sterol fluorescence in the perinuclear area and plotting the mean fluorescence as function of bleach time (see Fig. 9 in main text).
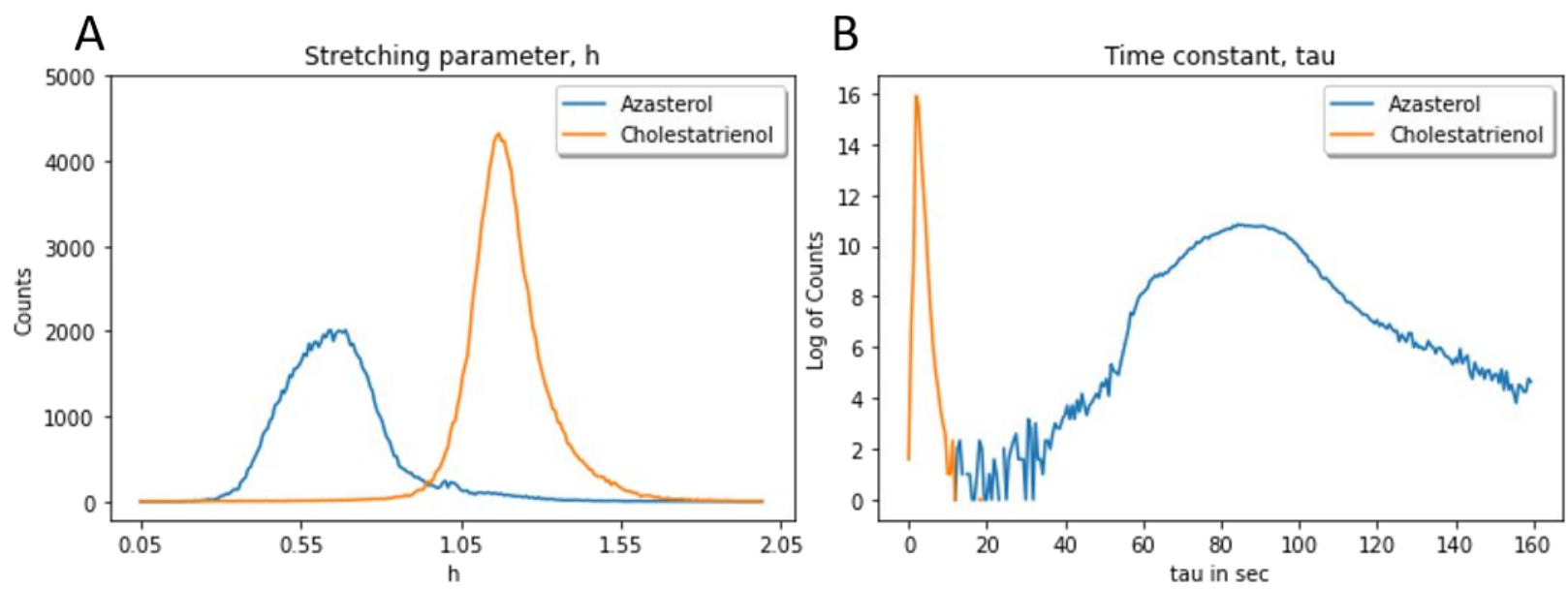

Figure S10. Pixel-wise bleaching analysis of azasterol and cholestatrienol in human fibroblasts. A stretched exponential decay function was fitted to each pixel of the bleaching stacks being acquired with UV filter sets. Histograms of stretching parameter h (panel A) and time constant tau (panel B) for entire cells are shown for azasterol and cholestatrienol. The stretching parameter $\mathrm{h}$ describes the shape of the bleaching curve, with $h<1$ for a compressed and $h>1$ for a stretched exponential bleaching kinetics, respectively. For $\mathrm{h}=1$, the bleaching would be mono-exponential. 


\section{Docking and Molecular Dynamics Simulations}

\section{Molecular Docking}

The initial protein structure coordinates for NPC1 (PDB ID: 3GKI, $1.80 \AA$ ) and NPC2 (PDB ID: 2HKA, $1.81 \AA$ ) were obtained from the RCSB Protein Data Bank. The protein structures were prepared with the Protein Preparation wizard in Schrödinger 2019. ${ }^{9}$ During protein preparation, water molecules were removed and then hydrogens were added to the protein and co-ligand. The energy of the protein structure was then minimized until the RMSD between the minimized structure and the starting structure reached $0.30 \AA$, using the OPLS3e force field. The ligand structures were prepared using the LigPrep module of Schrödinger 2019. LigPrep adds hydrogen atoms, generates tautomers, ionization states, ring conformations, and stereoisomers, and produces the energy minimized 3D structures using the OPLS3e force field. The possible ionization states were generated at target $\mathrm{pH} 7.0+/-2.0$ using the Epik module, and the compounds were desalted. For each input ligand structure, a single stereoisomer was generated by preserving the specified chirality. The molecular docking was performed using the GLIDE module of Schrödinger. ${ }^{9-12}$ The receptor grid was defined using ligand binding site information of the crystal structure. The extraprecision (XP) docking procedure was used for molecular docking, and all ligand poses and molecular interactions with key residues were analyzed.

\section{Molecular dynamics (MD) simulations and binding-free-energy calculations}

Molecular dynamics (MD) simulations and molecular mechanics/generalized Born surface area (MM/GBSA) calculations were performed in AMBER 18. The well validated ff14SB version of the all-atom AMBER force field was used to model the protein, the general AMBER force field (GAFF) was used for the organic ligand, and standard protocol described in the AMBER manual was followed. ${ }^{13-15}$ The initial coordinates for the protein atoms were taken from the X-ray structure of NPC1 (PDB ID: 3GKI, $1.80 \AA$ ) and NPC2 (PDB ID: 2HKA, $1.81 \AA$ ). Atom-centered partial charges were derived by using the AMBER antechamber program (restrained electrostatic potential methodology), after geometry optimization at the B3LYP/6-31G* level using Gaussian16. ${ }^{2}$ The ligand was placed by superposition of the binding mode predicted from computational studies. The pmemd module of AMBER 18 was used for the MD simulations. The protein atoms and all the water molecules of the crystal structure were surrounded by an octahedral box of TIP3P water molecules that extended at least $10 \AA$ from the protein. The molecular system was neutralized with $\mathrm{Na}^{+}$and $\mathrm{Cl}^{-}$counterions by LEaP. The particle mesh Ewald (PME) method was used for treating long-range electrostatic interactions with a $10 \AA$ cutoff for nonbonded van der Waals interactions and periodic boundary conditions. The time step was chosen to be $2 \mathrm{fs}$, and the SHAKE algorithm was used to constrain all bonds involving hydrogen atoms. The molecular systems were minimized in 1000 step increments that gradually reduced restraints on the atoms. The system was then heated to $300 \mathrm{~K}$ over $30 \mathrm{ps}$, followed by equilibration for $10 \mathrm{ps}$, followed by an NPT equilibration for $100 \mathrm{ps}$. The temperature and pressure were maintained using a Langevin thermostat and a Berendsen barostat with isotropic scaling, respectively. Each molecular system was simulated for $100 \mathrm{~ns}$. The trajectories were analyzed using the CPPTRAJ module of AmberTools18.

The binding free energy $\left(\Delta \mathrm{G}_{\text {bind }}\right)$ of the receptor/ligand complex was calculated using MM/GBSA methodology. A series of snapshots produced by the MD simulation were used for calculation of binding free energy. The exterior dielectric constant was set to 80, and the solute dielectric constant was set to 1 . The total binding free energy can be calculated according to the equation: 


$$
\begin{aligned}
\Delta \mathrm{G}_{\text {bind }} & =\Delta \mathrm{G}_{\mathrm{gas}}+\Delta \mathrm{G}_{\mathrm{solv}}-\mathrm{T} \Delta \mathrm{S} \\
& =\Delta \mathrm{E}_{\mathrm{MM}}+\Delta \mathrm{G}_{\mathrm{GB}}+\Delta \mathrm{G}_{\mathrm{SA}}-\mathrm{T} \Delta \mathrm{S} \\
& =\Delta \mathrm{E}_{\mathrm{vdw}}+\mathrm{E}_{\mathrm{ele}}+\mathrm{G}_{\mathrm{GB}}+\Delta \mathrm{G}_{\mathrm{SA}}-\mathrm{T} \Delta \mathrm{S}
\end{aligned}
$$

where $\Delta \mathrm{E}_{\mathrm{MM}}$ represents the gas-phase interaction energy between receptor and ligand [including van der Waals energy contribution $\left(\Delta \mathrm{E}_{\mathrm{vdw}}\right)$ and electrostatic energy contribution $\left.\left(\Delta \mathrm{E}_{\mathrm{ele}}\right)\right] ; \Delta \mathrm{G}_{\mathrm{GB}}$ and $\Delta \mathrm{G}_{\mathrm{SA}}$ denotes polar and nonpolar components of the desolvation free energy, respectively; $\mathrm{T} \Delta \mathrm{S}$ represents the conformational entropy contribution at temperature $\mathrm{T}$. Here, $\Delta \mathrm{G}_{\mathrm{GB}}$ was determined by the Generalized-Born approximation model, while $\Delta \mathrm{G}_{\mathrm{SA}}$ was calculated using 'LCPO' surface areas. The entropy term was not considered due to high computational cost and low prediction accuracy.

Table S5. Summary of docking score results and components of binding free energy $(\mathrm{kcal} / \mathrm{mol})$

\begin{tabular}{|c|c|c|c|c|c|c|}
\hline Complex & $\begin{array}{l}\text { Docking } \\
\text { Score }\end{array}$ & ${ }^{\mathrm{a}} \Delta \mathbf{E}_{\mathrm{vdw}}$ & ${ }^{\mathrm{b}} \Delta \mathbf{E}_{\text {elec }}$ & ${ }^{\mathrm{c}} \Delta \mathbf{G}_{\mathrm{GB}}$ & ${ }^{\mathrm{d}} \Delta \mathbf{G}_{\mathrm{SA}}$ & $\begin{array}{l}\text { MM-GBSA } \\
\text { s }_{\text {Total }}\end{array}$ \\
\hline $\begin{array}{l}\text { PC1- } \\
\text { holesterol }\end{array}$ & 13.55 & $-66.50(0.02)$ & $-11.76(0.02)$ & $20.93(0.01)$ & $-7.78(0.01)$ & $-66.50(0.03)$ \\
\hline $\begin{array}{l}\text { PC2- } \\
\text { holesterol }\end{array}$ & 10.76 & $-51.46(0.02)$ & $-0.31(0.01)$ & $9.13(0.01)$ & $-6.19(0.01)$ & $-48.82(0.02)$ \\
\hline $\begin{array}{l}\mathrm{C} 1- \\
\text { asterol }\end{array}$ & -10.78 & $-56.87(0.03)$ & $-15.63(0.03)$ & $26.78(0.02)$ & $-7.16(0.01)$ & $-52.88(0.03)$ \\
\hline $\begin{array}{l}\text { NPC2- } \\
\text { Azasterol }\end{array}$ & -7.18 & $-50.43(0.03)$ & $-1.09(0.01)$ & $12.72(0.02)$ & $-6.13(0.01)$ & $-44.93(0.03)$ \\
\hline \multicolumn{7}{|c|}{$\begin{array}{l}{ }^{\mathrm{a}} \Delta \mathrm{E}_{\mathrm{Vdw}} \text { : van der Waals energy. } \\
{ }^{\mathrm{b}} \Delta \mathrm{E}_{\mathrm{ele}} \text { : Electrostatic energy. } \\
{ }^{\mathrm{c}} \Delta \mathrm{G}_{\mathrm{GB}}: \text { Electrostatic contribution to the solvation free energy calculated by Generalized Born (GB). } \\
{ }^{\mathrm{d}} \Delta \mathrm{G}_{\mathrm{SA}}: \text { Nonpolar contribution to the solvation free energy calculated by LCPO method. }\end{array}$} \\
\hline
\end{tabular}
calculated for the binding of cholesterol/azasterol (1) with NPC1 and NPC2 using MM-GBSA method. 


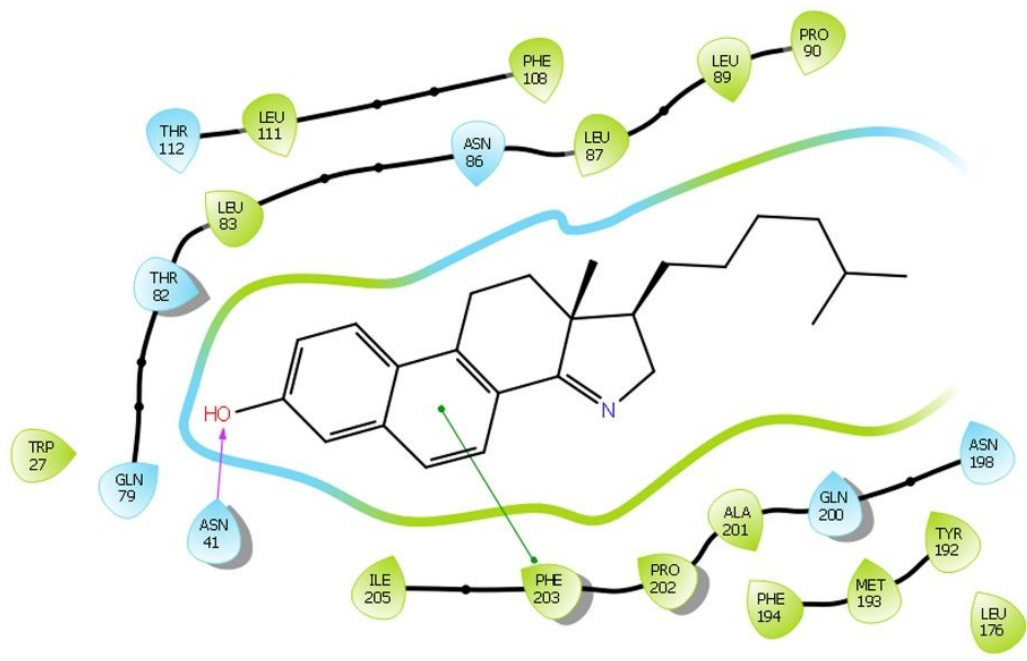

Figure S11: Protein ligand interactions observed during the molecular docking of azasterol (1) in NPC1. H-bonding and $\pi-\pi$ interactions are indicated in purple and green colored lines, respectively.
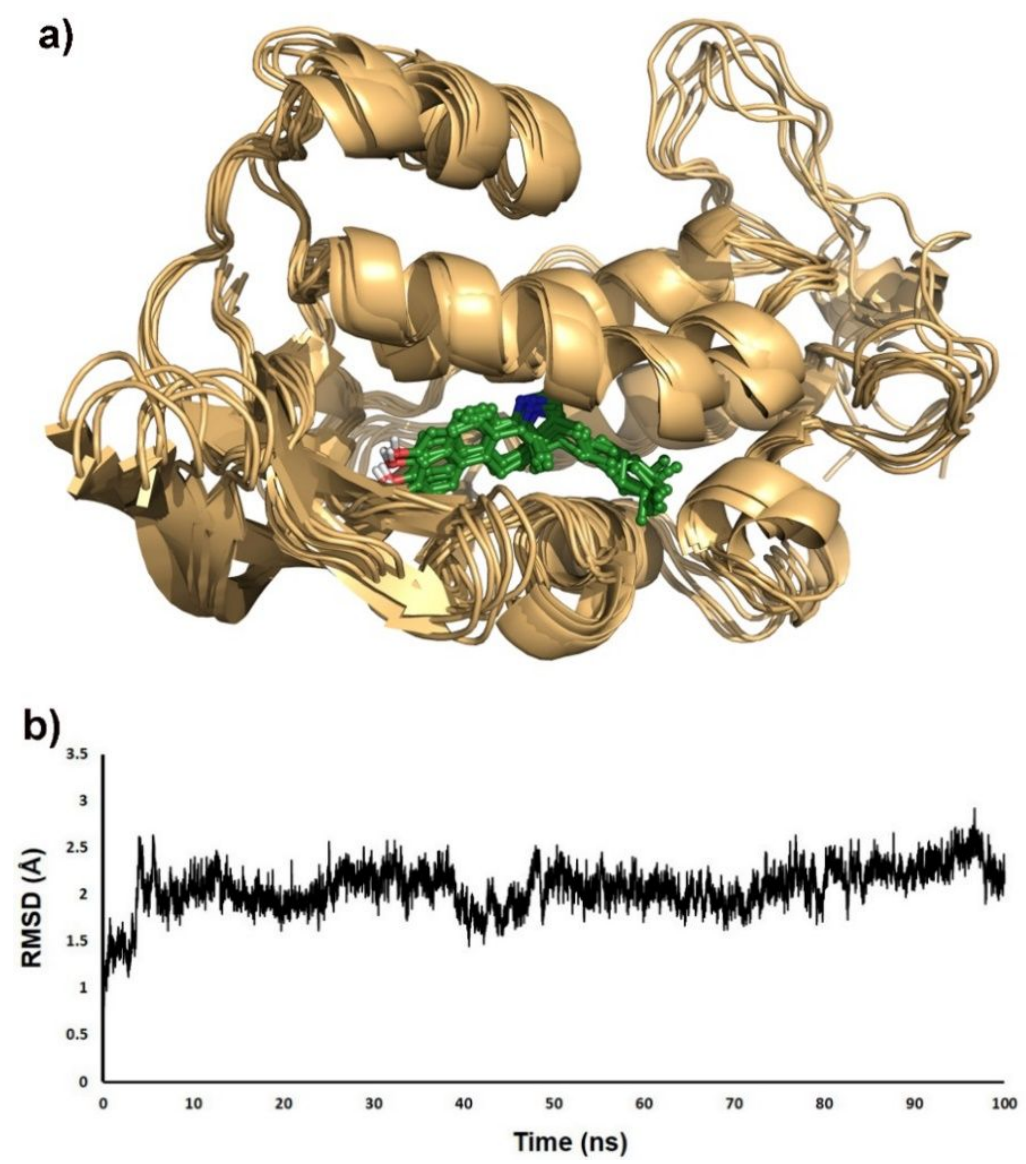

Figure S12: a) Overlay of MD simulations frames, b) root mean square deviation (RMSD) plot of the backbone atoms for azasterol-NPC1 complex. 

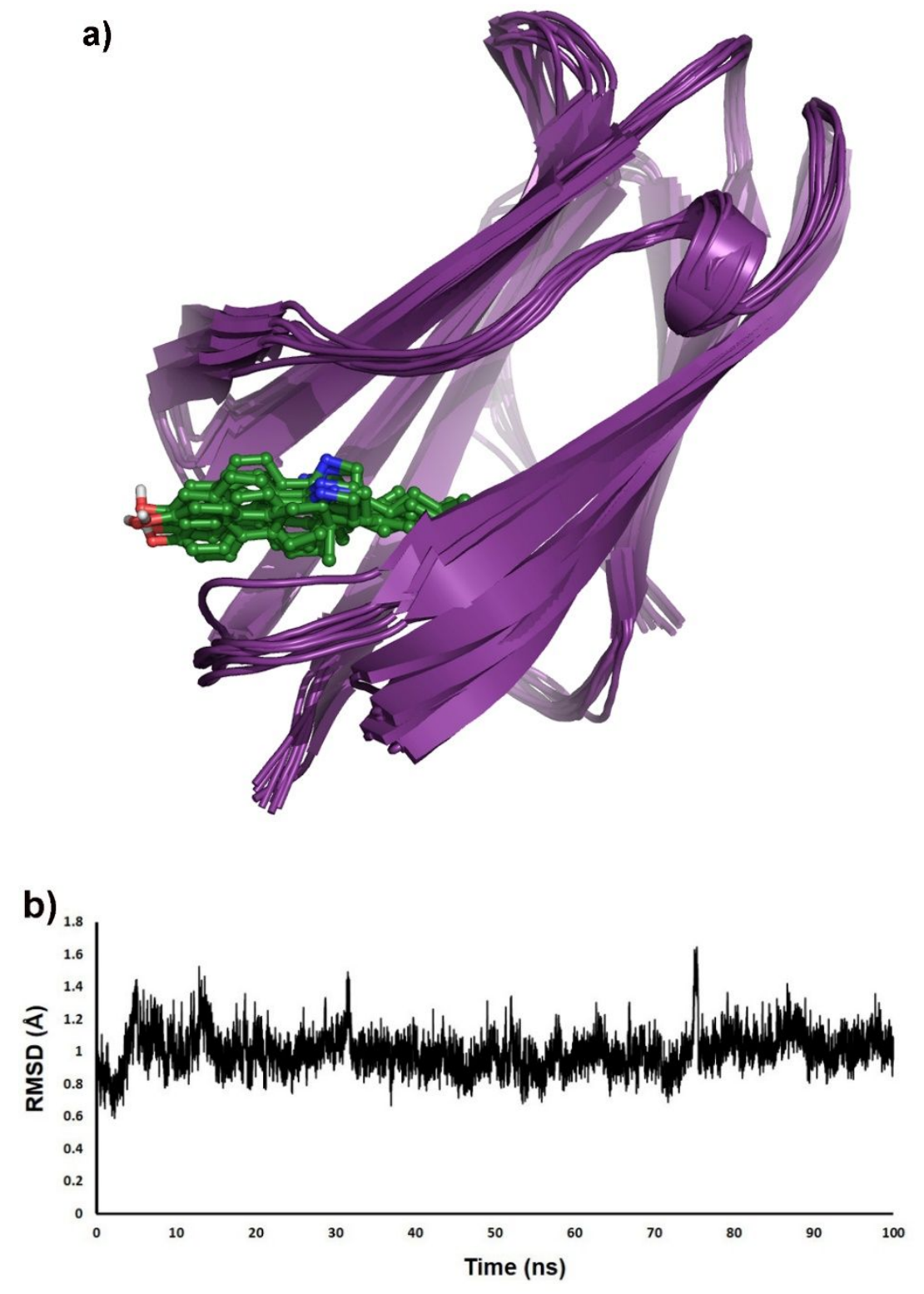

Figure S13: a) Overlay of MD simulations frames, b) RMSD plot of the backbone atoms for azasterol-NPC2 complex. 

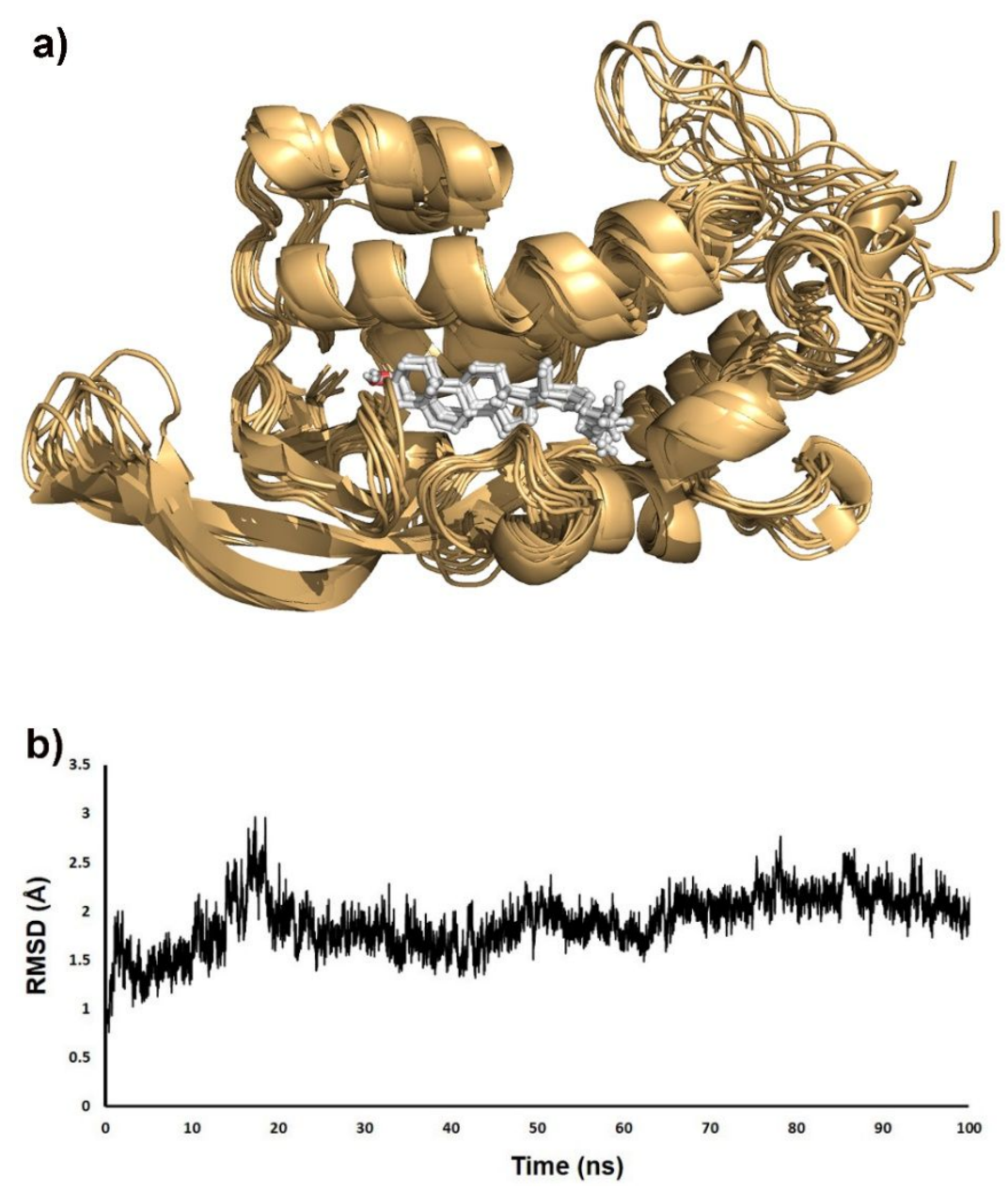

Figure S14: a) Overlay of MD simulations frames, b) RMSD plot of the backbone atoms for cholesterol-NPC1 complex. 

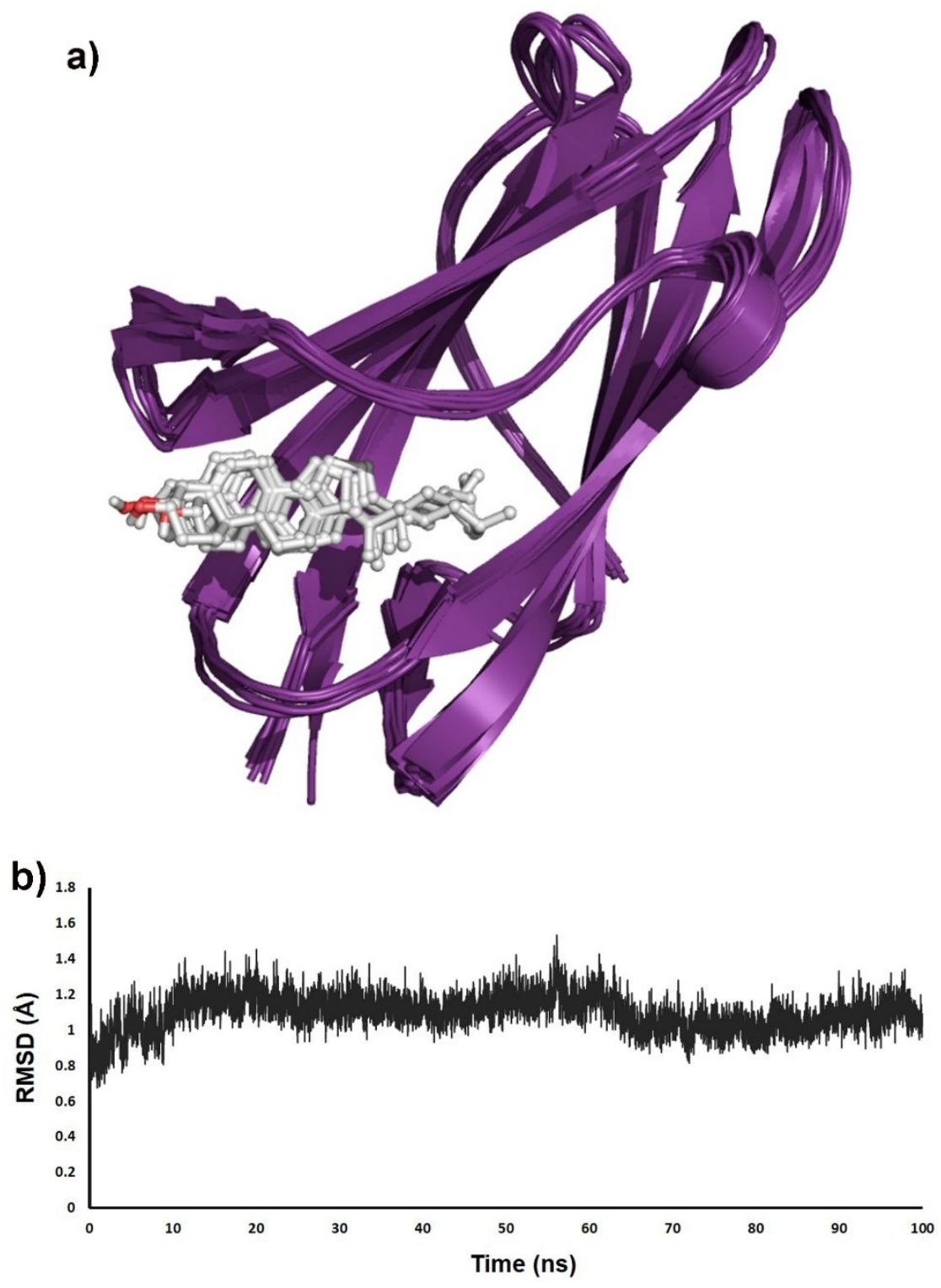

Figure S15: a) Overlay of MD simulations frames, b) RMSD plot of the backbone atoms for cholesterol-NPC 2 complex. 


\section{Spectra}

${ }^{1} \mathrm{H} \mathrm{NMR}\left(\mathrm{CDCl}_{3}, 400 \mathrm{MHz}\right)$

2

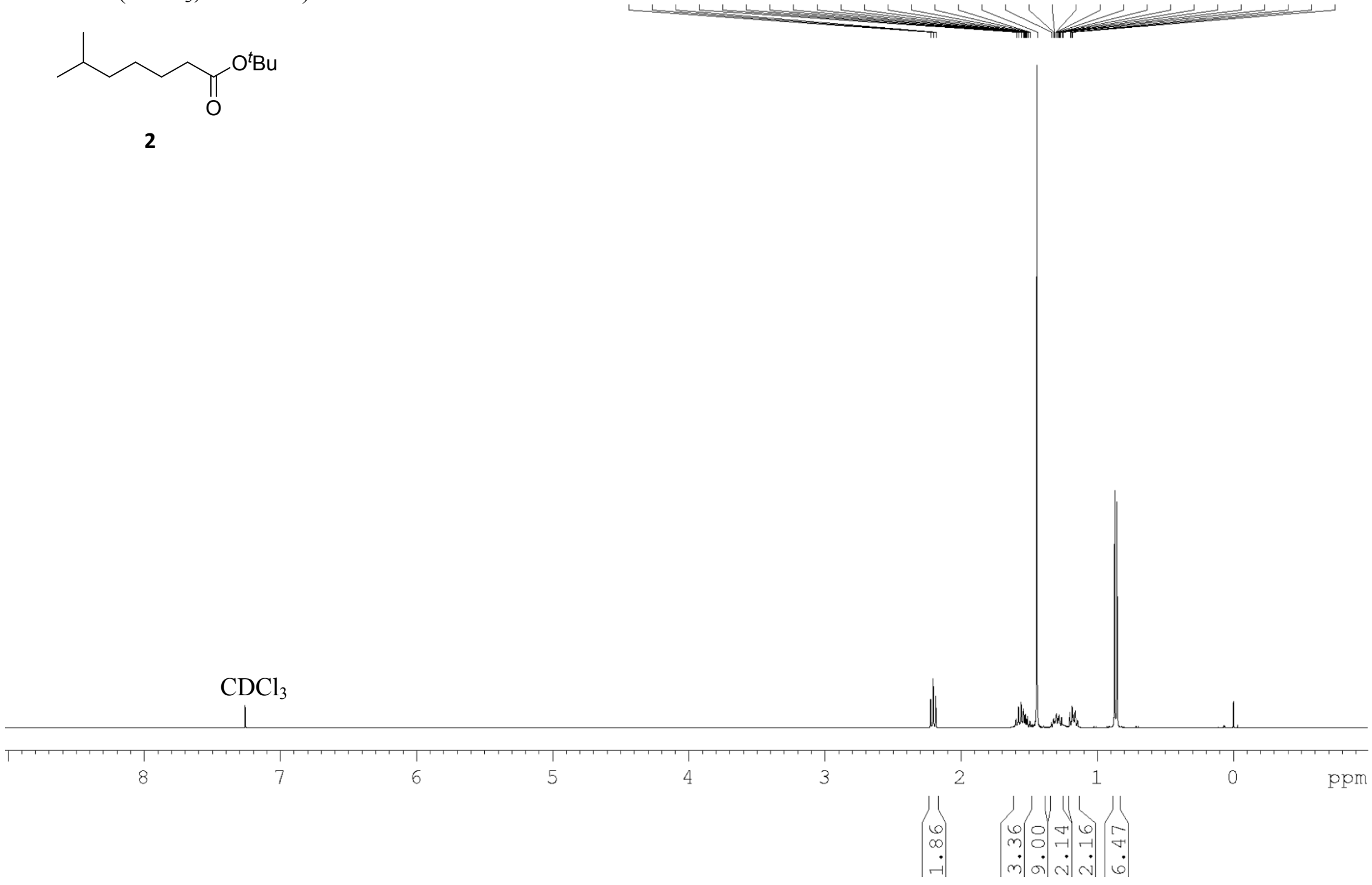

NOm

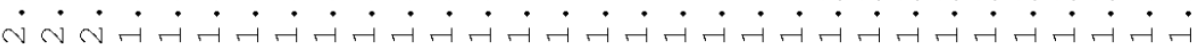


${ }^{13} \mathrm{C}\left\{{ }^{1} \mathrm{H}\right\} \mathrm{NMR}\left(\mathrm{CDCl}_{3}, 201 \mathrm{MHz}\right)$
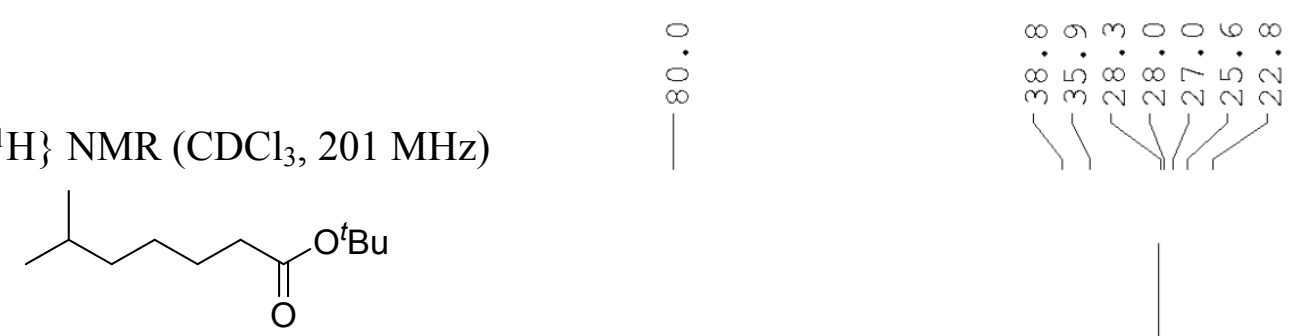

2

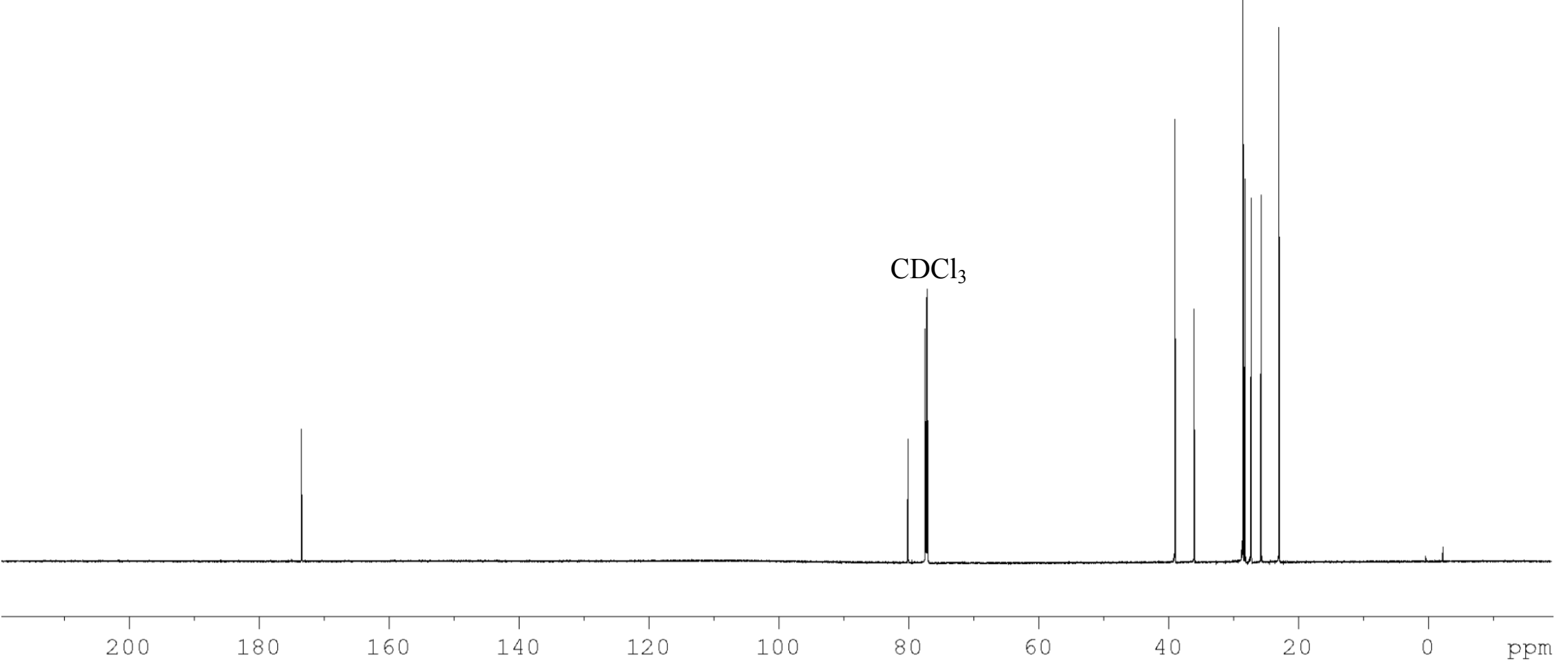




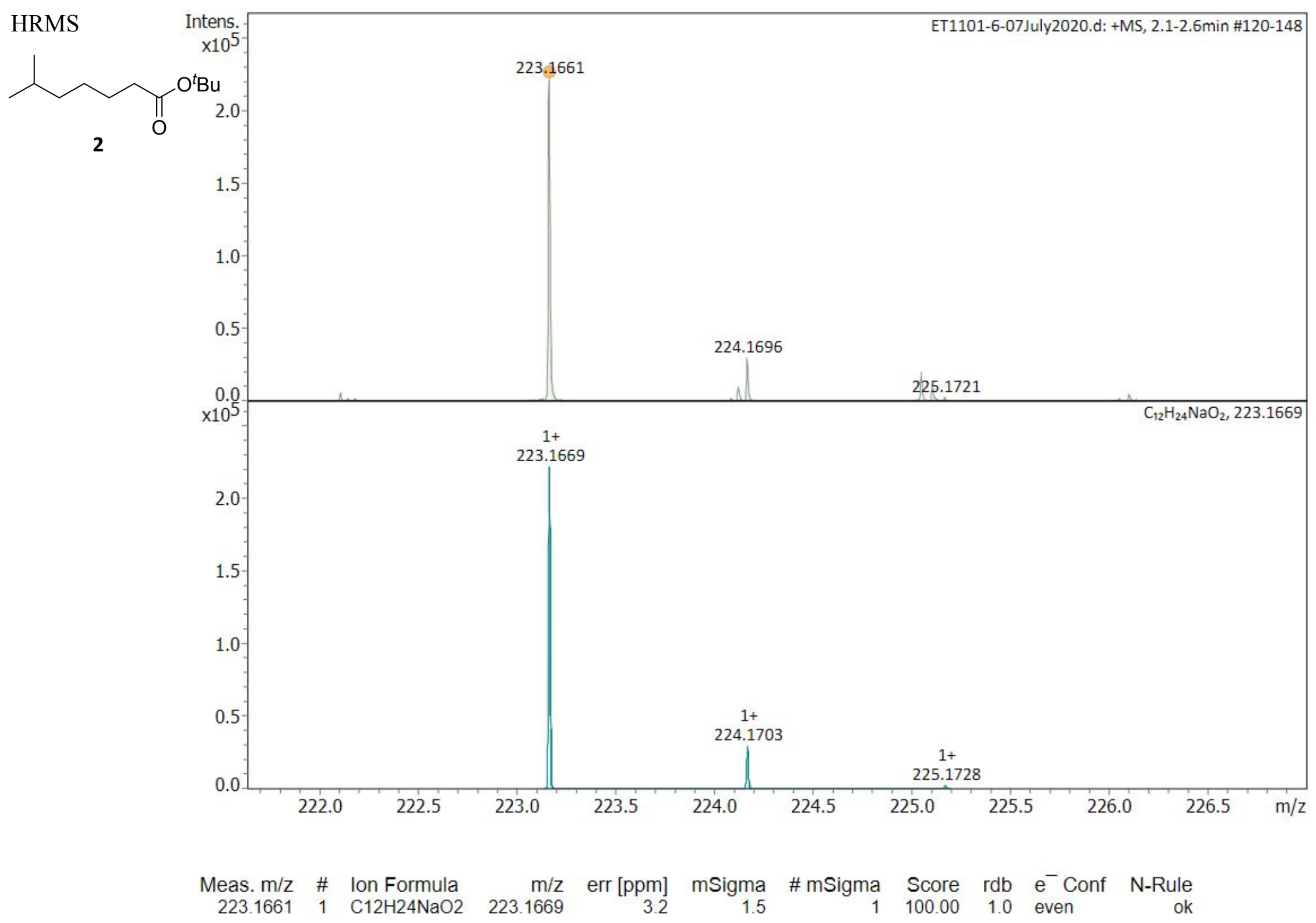


FTIR (ATR)

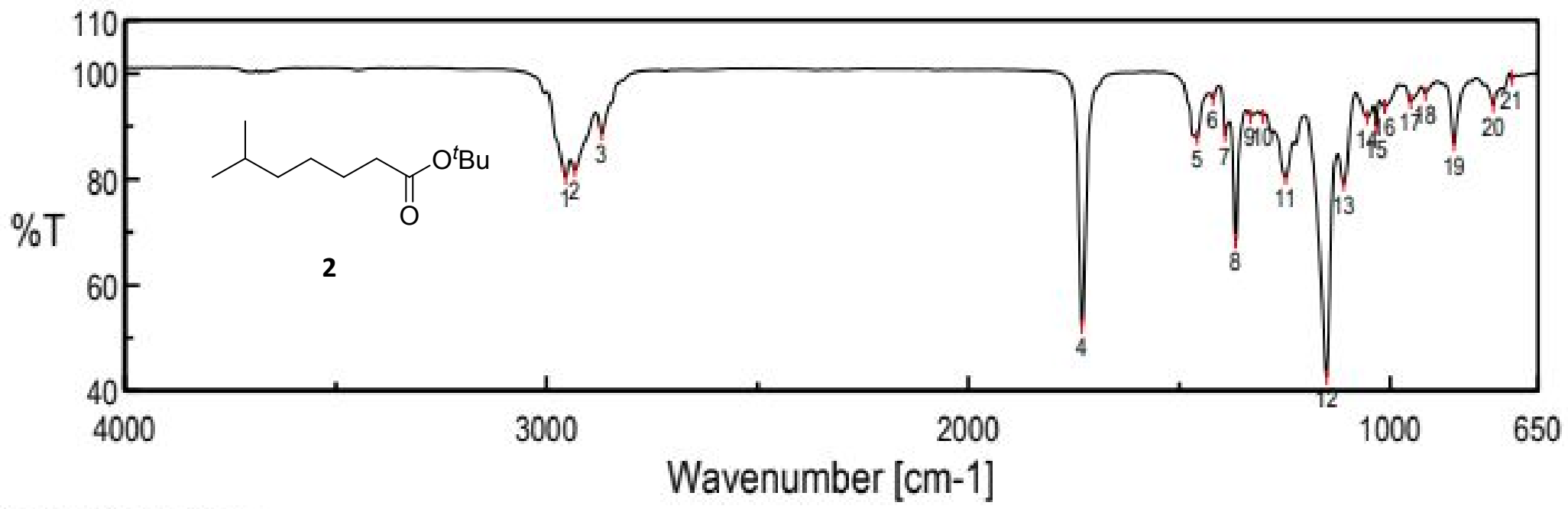

[Result of Peak Picking]

$\begin{array}{lllllllll}\text { No. } & \text { Position } & \text { Intensity } & \text { No. } & \text { Position } & \text { Intensity } & \text { No. } & \text { Position } & \text { Intensity } \\ 1 & 2955.38 & 80.2619 & 2 & 2933.2 & 81.7906 & 3 & 2869.56 & 88.626 \\ 4 & 1730.8 & 52.1573 & 5 & 1457.92 & 87.8445 & 6 & 1420.32 & 95.197 \\ 7 & 1391.39 & 88.37 & 8 & 1366.32 & 68.2783 & 9 & 1331.61 & 91.7867 \\ 10 & 1301.72 & 91.7902 & 11 & 1247.72 & 80.3157 & 12 & 1151.29 & 42.4377 \\ 13 & 1109.83 & 79.0653 & 14 & 1053.91 & 91.6433 & 15 & 1032.69 & 89.8357 \\ 16 & 1013.41 & 93.6375 & 17 & 951.698 & 94.6018 & 18 & 916.986 & 96.0397 \\ 19 & 848.525 & 86.6246 & 20 & 754.995 & 93.8591 & 21 & 711.604 & 99.0475\end{array}$




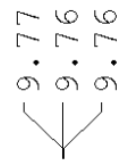

${ }^{1} \mathrm{H} \mathrm{NMR}\left(\mathrm{CDCl}_{3}, 400 \mathrm{MHz}\right)$

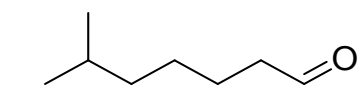

3

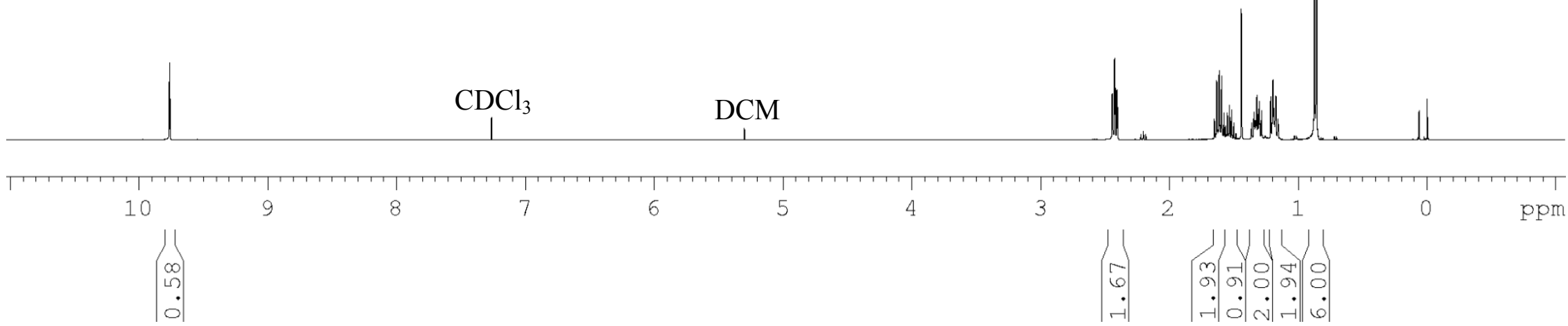




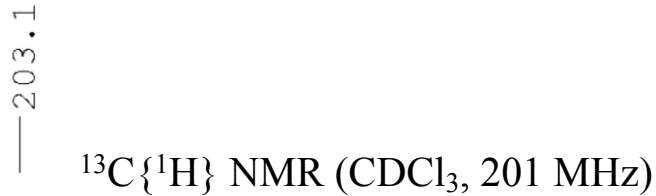

$\sim^{\infty}$

3

ํ. $\because \cdots \infty$

$\dot{\rightarrow} \infty \dot{\sim} \dot{\sim} \dot{N}$

$\mathrm{CDCl}_{3}$

,

111

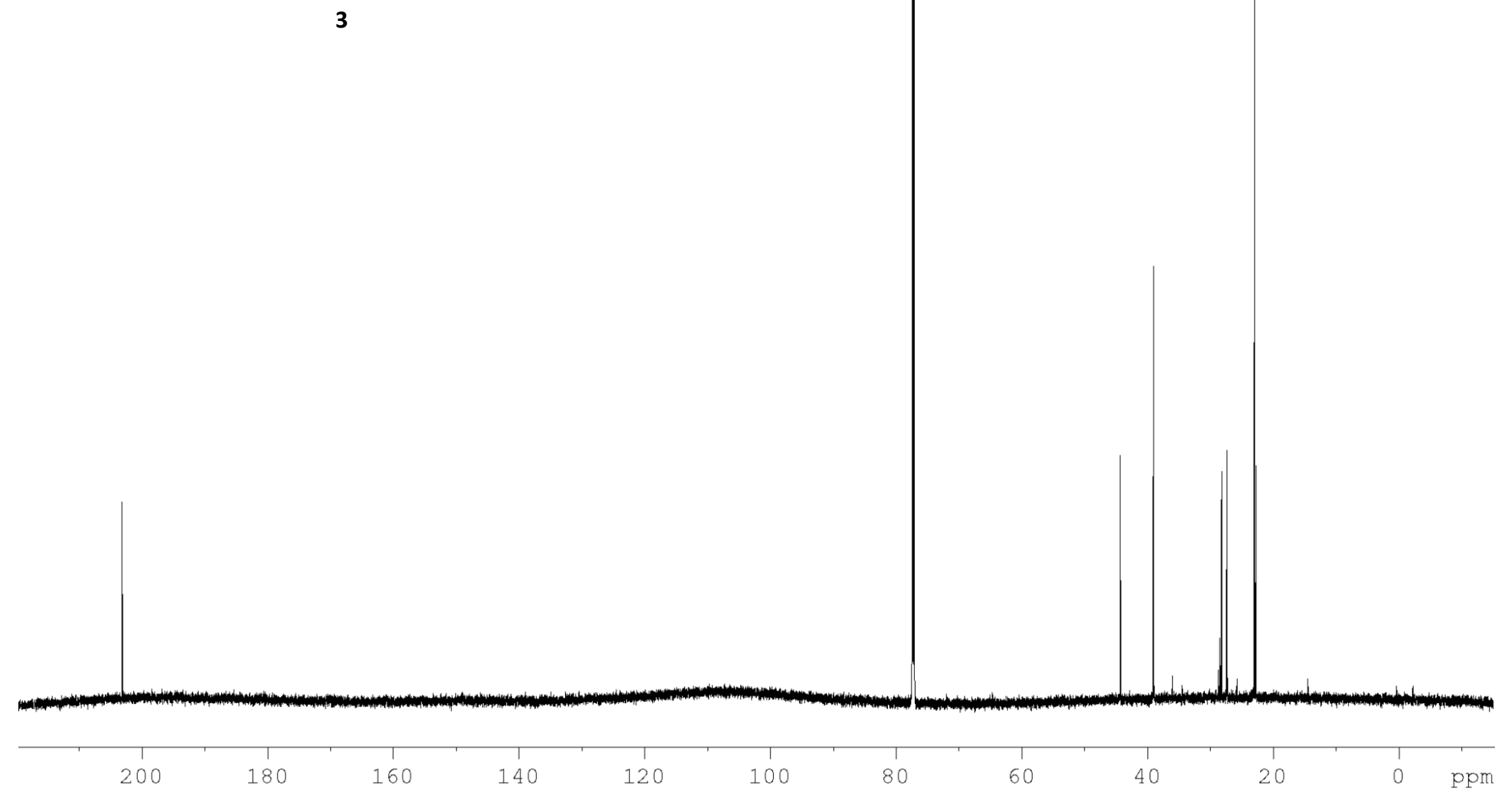


FTIR (ATR)

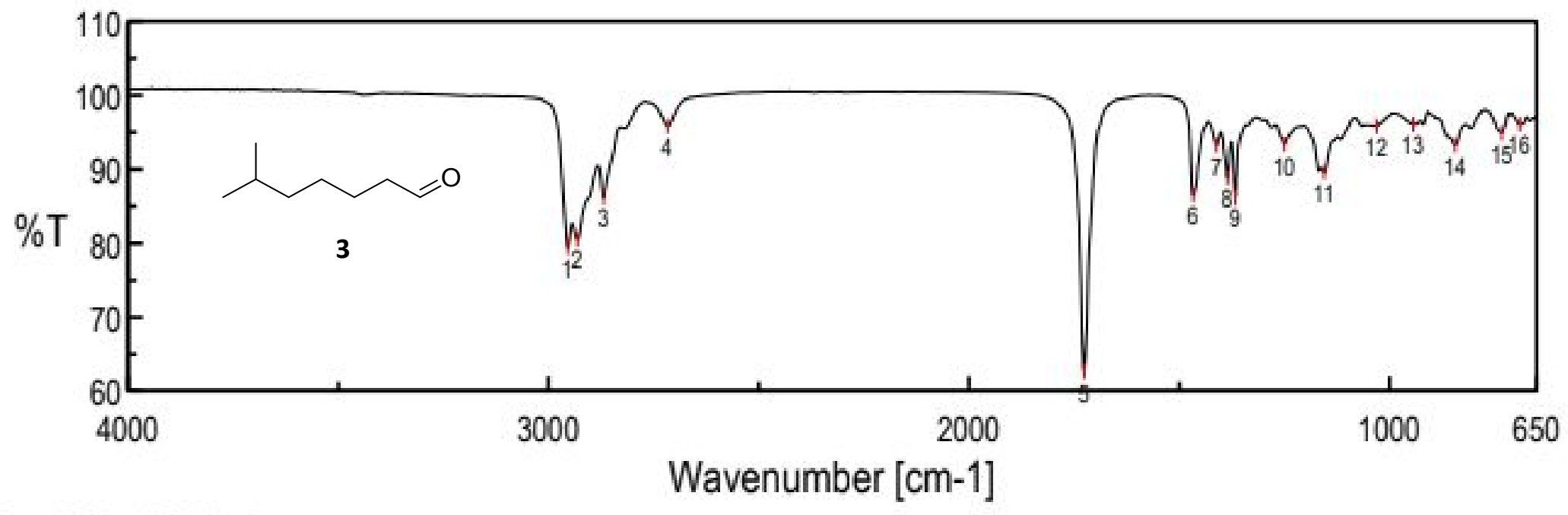

[ Result of Peak Picking ]

$\begin{array}{lllllllll}\text { No. } & \text { Position } & \text { Intensity } & \text { No. } & \text { Position } & \text { Intensity } & \text { No. } & \text { Position } & \text { Intensity } \\ 1 & 2954.41 & 79.381 & 2 & 2931.27 & 80.4875 & 3 & 2868.59 & 86.1379 \\ 4 & 2716.25 & 95.7876 & 5 & 1725.98 & 62.6166 & 6 & 1466.6 & 86.4869 \\ 7 & 1411.64 & 93.2658 & 8 & 1385.6 & 88.8582 & 9 & 1366.32 & 86.083 \\ 10 & 1249.65 & 93.3352 & 11 & 1155.15 & 89.6047 & 12 & 1030.77 & 95.7532 \\ 13 & 943.985 & 96.0143 & 14 & 843.704 & 93.2465 & 15 & 732.817 & 94.9084 \\ 16 & 690.391 & 96.0495 & & & & & & \end{array}$


${ }^{1} \mathrm{H} \mathrm{NMR}\left(\mathrm{CDCl}_{3}, 400 \mathrm{MHz}\right)$

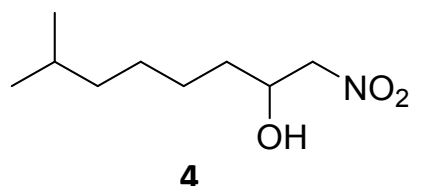

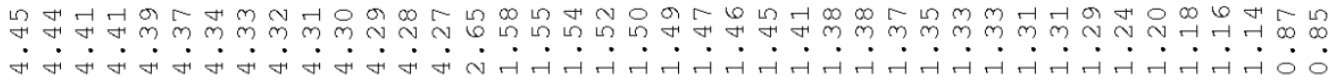
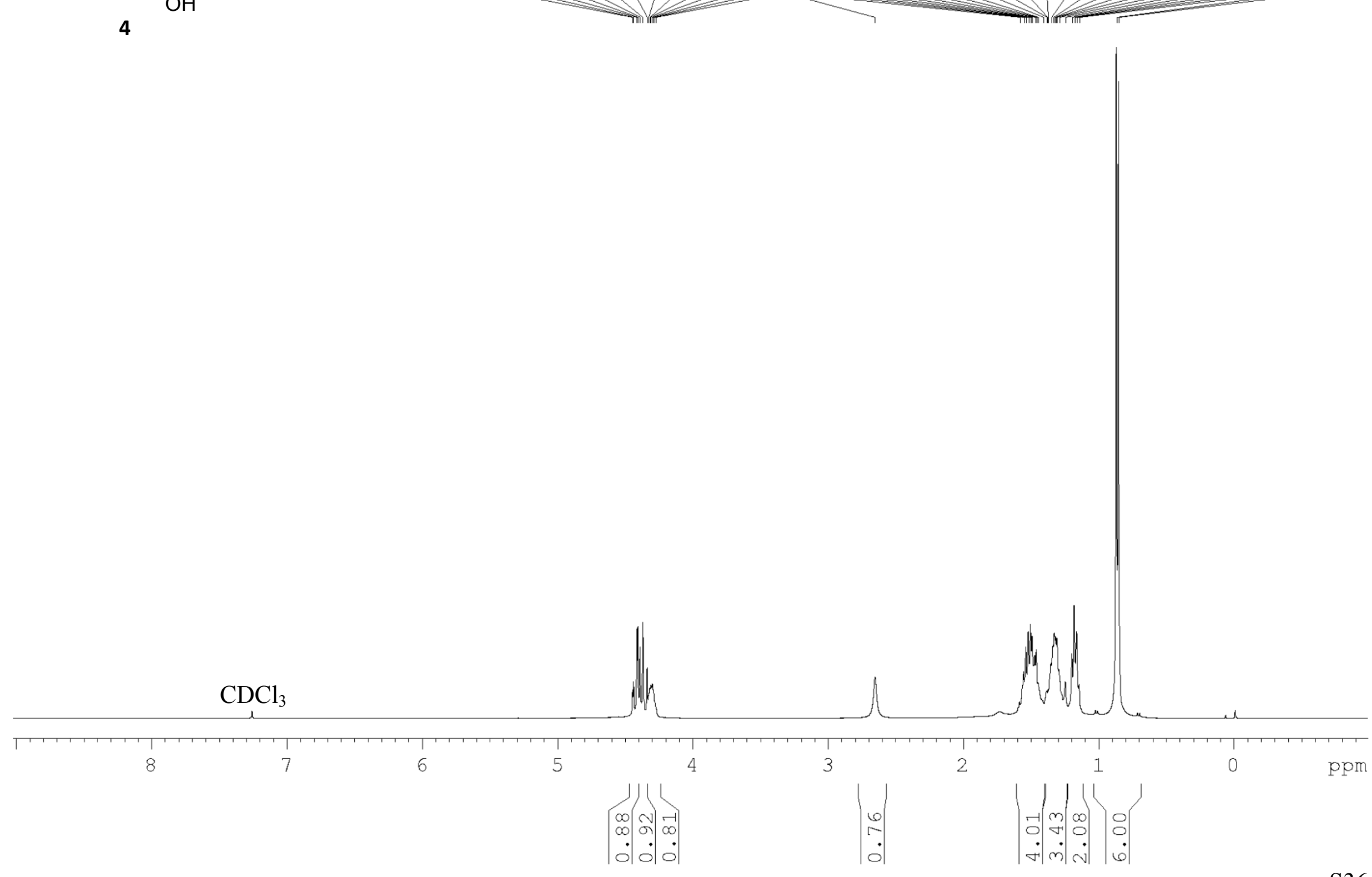


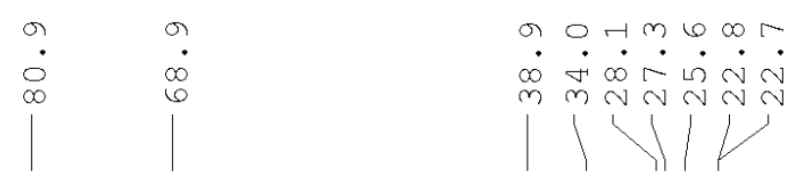

${ }^{13} \mathrm{C}\left\{{ }^{1} \mathrm{H}\right\}$ NMR $\left(\mathrm{CDCl}_{3}, 101 \mathrm{MHz}\right)$

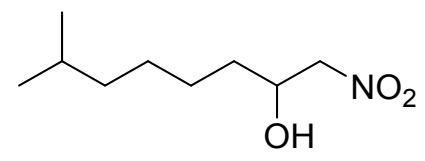

4

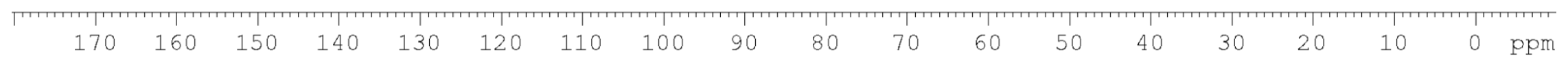


FTIR (ATR)

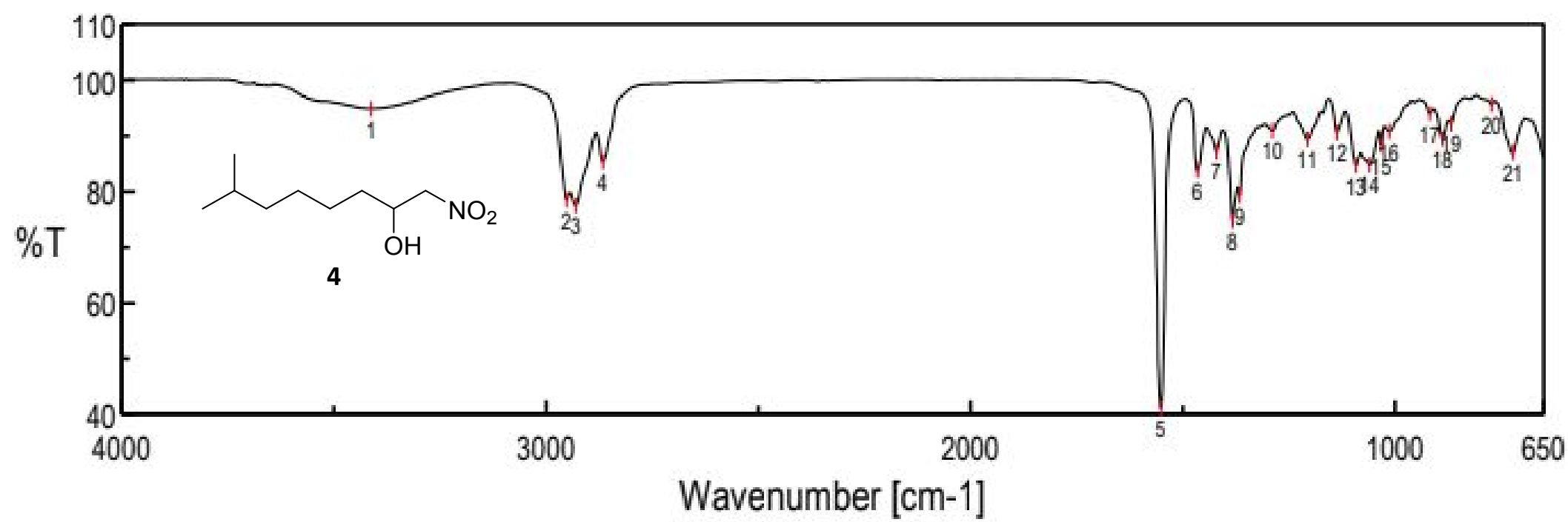

[ Result of Peak Picking ]

$1 \quad 3411.46 \quad 94.824$

$\begin{array}{lll}5 & 1551.45 & 41.0684\end{array}$

$13 \quad 1092.48 \quad 84.6733$

$\begin{array}{lll}17 & 917.95 & 93.9499\end{array}$

$21 \quad 722.211 \quad 86.9622$

$\begin{array}{lll}\text { No. } & \text { Position } & \text { Intensity } \\ 2 & 2952.48 & 78.5139 \\ 6 & 1465.63 & 83.763 \\ 10 & 1289.18 & 90.8999 \\ 14 & 1059.69 & 84.7291 \\ 18 & 889.023 & 89.432\end{array}$

$\begin{array}{lll}\text { No. } & \text { Position } & \text { Intensity } \\ 3 & 2930.31 & 77.4537 \\ 7 & 1420.32 & 87.1322 \\ 11 & 1206.26 & 89.3439 \\ 15 & 1032.69 & 88.3434 \\ 19 & 867.81 & 92.1277\end{array}$

$\begin{array}{lll}\text { No. } & \text { Position } & \text { Intensity } \\ 4 & 2866.67 & 85.3314 \\ 8 & 1383.68 & 74.7781 \\ 12 & 1136.83 & 90.5652 \\ 16 & 1014.37 & 90.728 \\ 20 & 772.351 & 95.6176\end{array}$




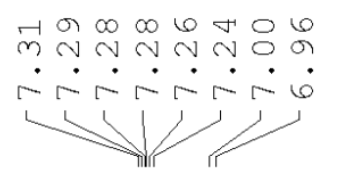

${ }^{1} \mathrm{H} \mathrm{NMR}\left(\mathrm{CDCl}_{3}, 400 \mathrm{MHz}\right)$

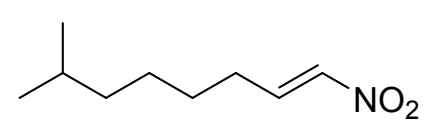

5

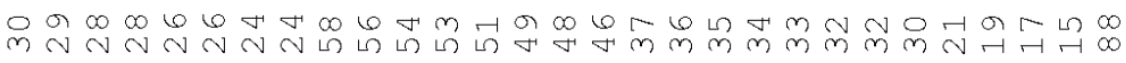

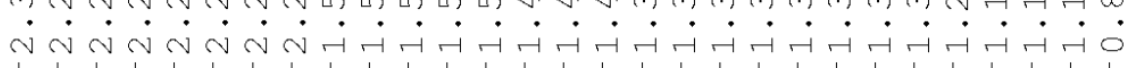

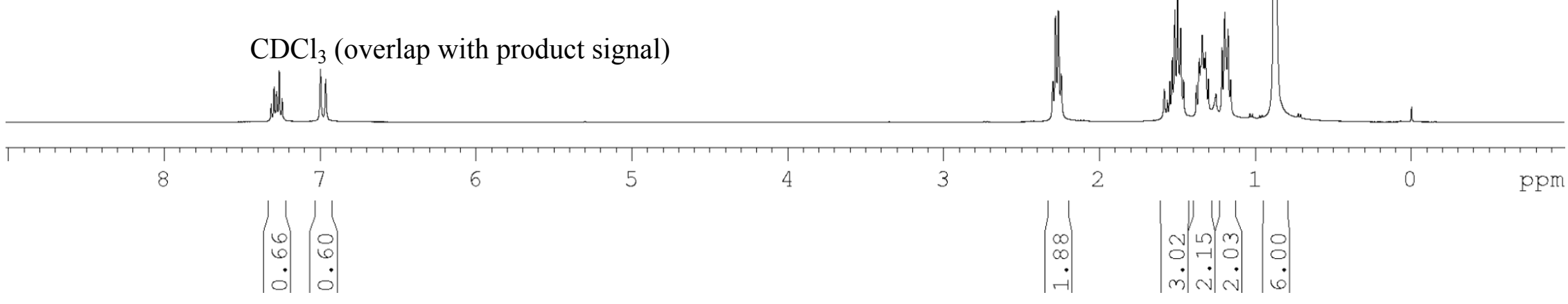




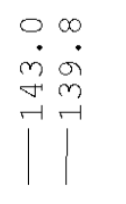

${ }^{13} \mathrm{C}\left\{{ }^{1} \mathrm{H}\right\}$ NMR $\left(\mathrm{CDCl}_{3}, 101 \mathrm{MHz}\right)$

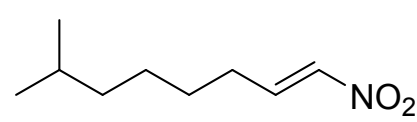

5

. . . . . .

$\infty \quad \infty \quad \infty \quad \infty \quad r$
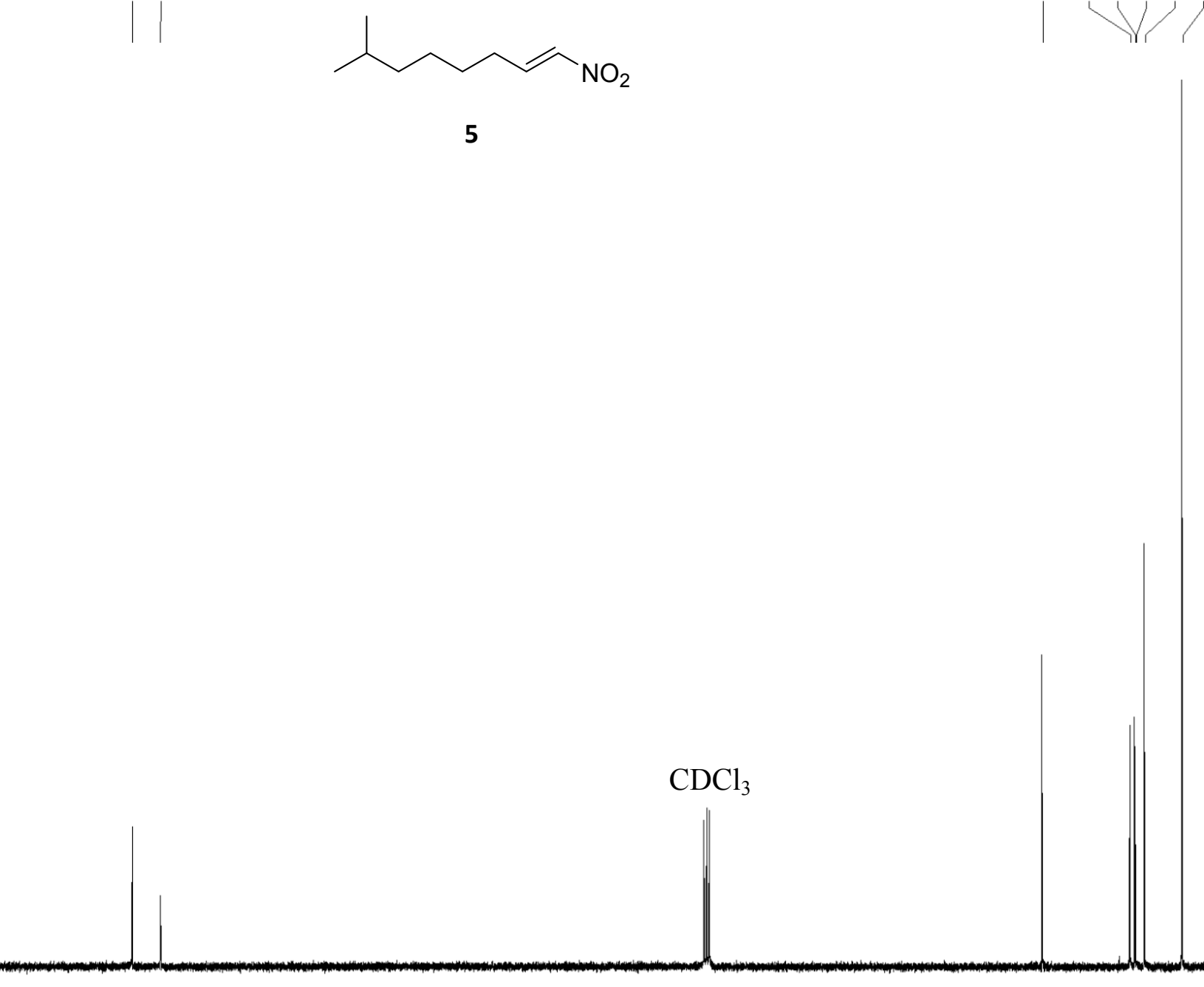

$\begin{array}{lll}170 & 160 & 150\end{array}$

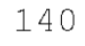

130

120

110

100

90
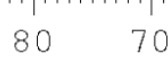

60

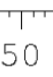

40

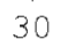

20 
LC

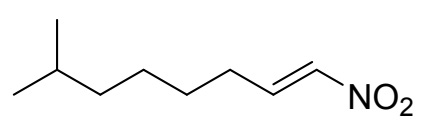

5

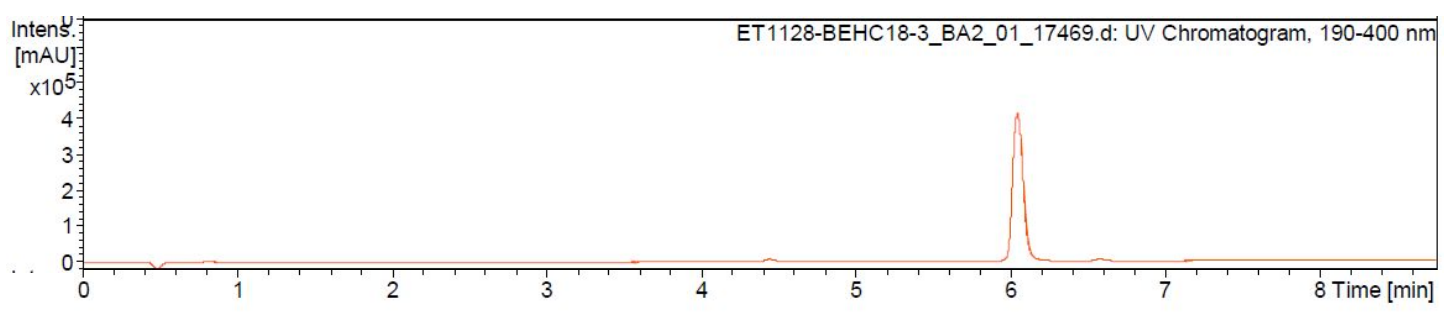

HRMS
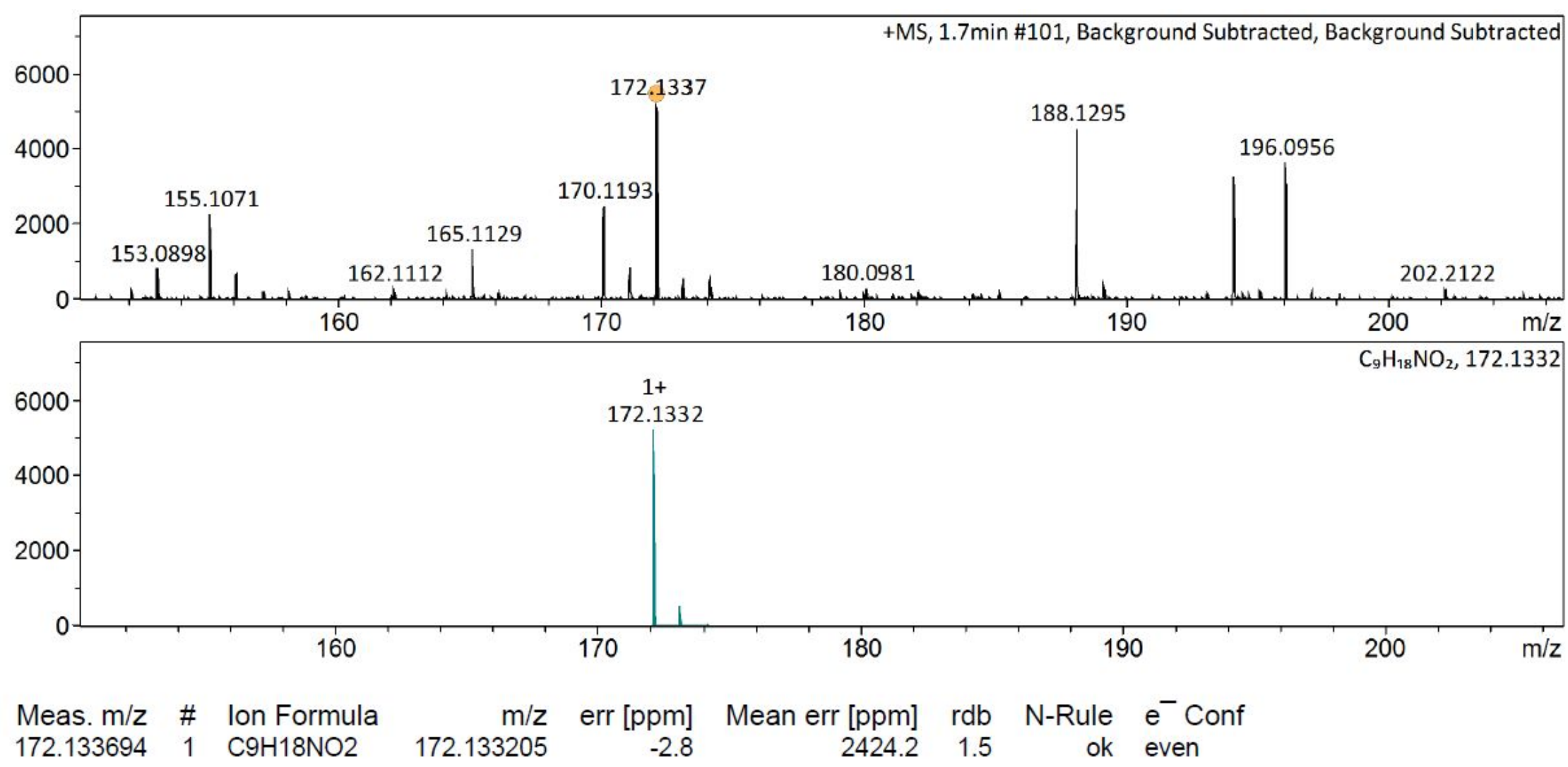
FTIR (ATR)

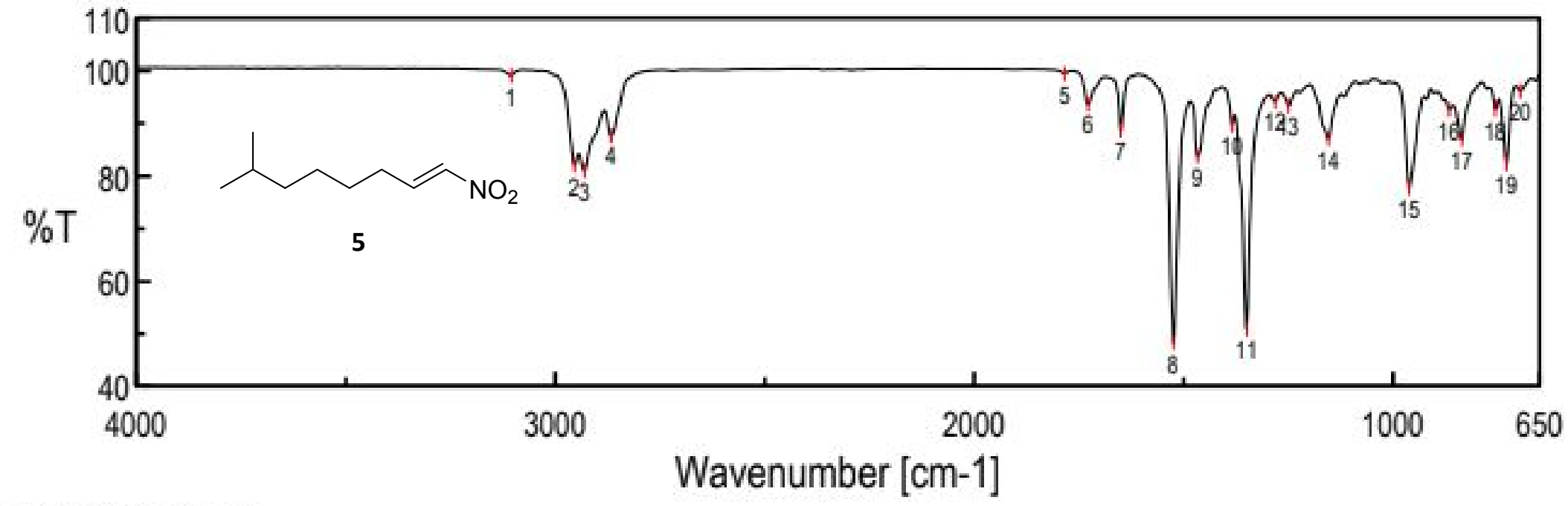

[Result of Peak Picking]

No. Position Intensity

$\begin{array}{lll}1 & 3104.83 & 99.0641\end{array}$

No. Position

$\begin{array}{ll}3104.83 & 99.0641 \\ 2866.67 \quad 87.6288\end{array}$

$\begin{array}{lll}7 & 1649.8 & 88.5996\end{array}$

$\begin{array}{lll}10 & 1384.64 & 89.6807\end{array}$

$\begin{array}{lll}13 & 1249.65 & 93.3057\end{array}$

$\begin{array}{lll}16 & 865.882 & 92.5843\end{array}$

$\begin{array}{lll}19 & 729.925 & 82.3052\end{array}$

$\begin{array}{lll}2 & 2954.41 & 82.0429 \\ 5 & 1783.83 & 99.4465 \\ 8 & 1524.45 & 48.239 \\ 11 & 1349.93 & 50.8517 \\ 14 & 1154.19 & 87.0646 \\ 17 & 835.99 & 86.9503 \\ 20 & 696.177 & 96.137\end{array}$

$\begin{array}{lll}\text { No. } & \text { Position } & \text { Intensity } \\ 3 & 2930.31 & 81.0115 \\ 6 & 1727.91 & 93.6187 \\ 9 & 1466.6 & 83.7343 \\ 12 & 1281.47 & 94.1124 \\ 15 & 961.341 & 77.7333 \\ 18 & 755.959 & 92.6587\end{array}$




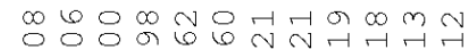

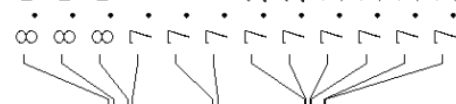

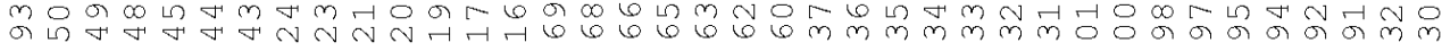

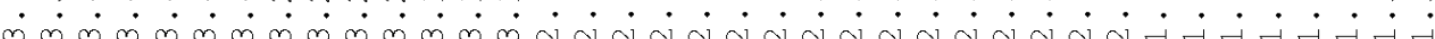

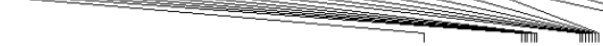

${ }^{1} \mathrm{H} \mathrm{NMR}\left(\mathrm{CDCl}_{3}, 400 \mathrm{MHz}\right)$
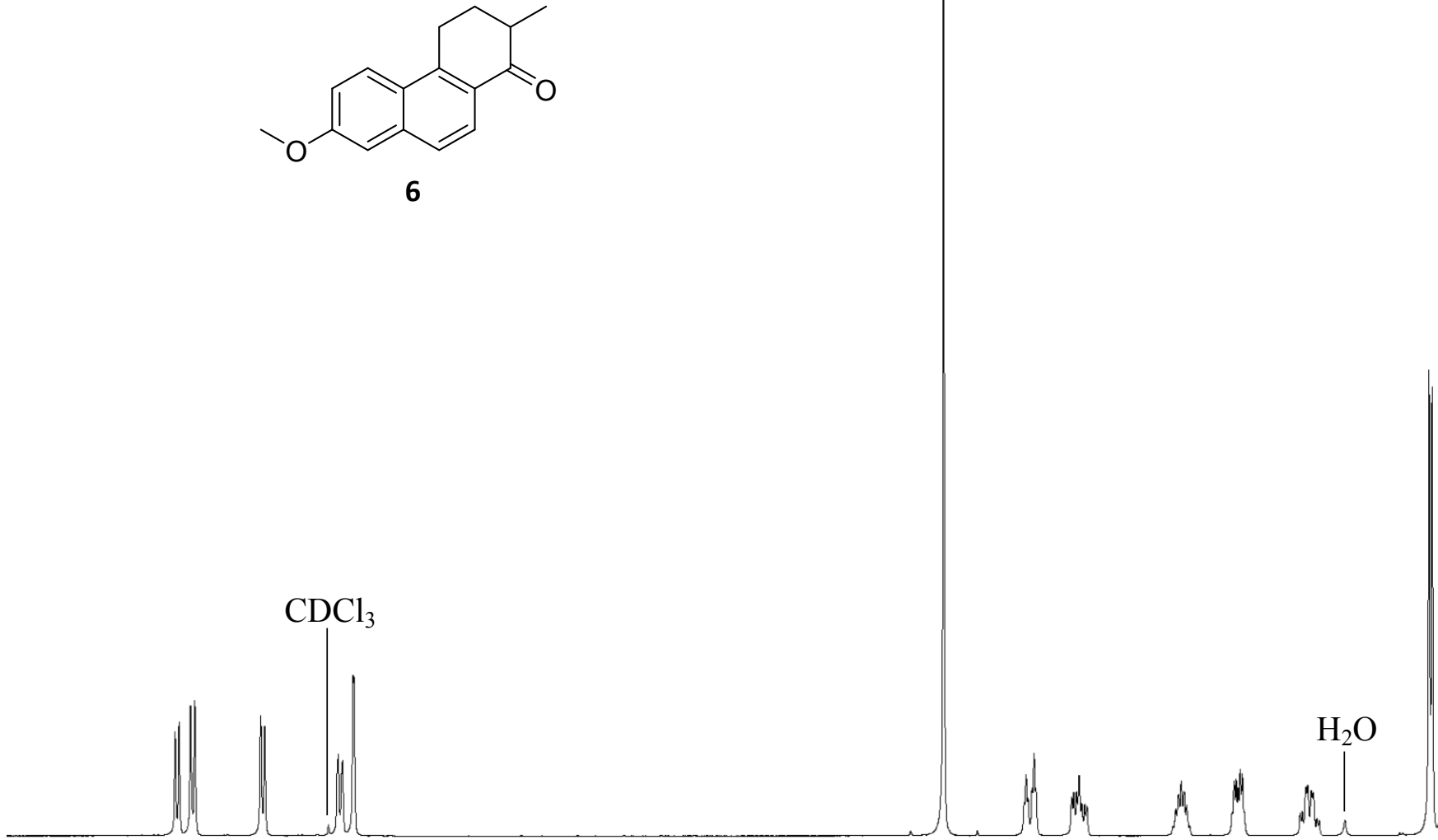

8

6

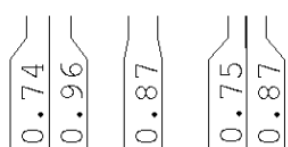

$\left|\begin{array}{c|c|c|}4 & \\ 0 & 0 & 0 \\ 0\end{array}\right|$

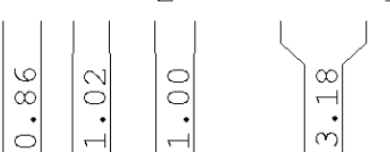




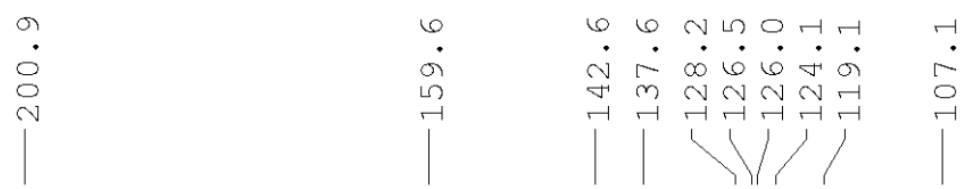

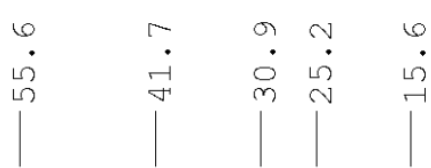

${ }^{13} \mathrm{C}\left\{{ }^{1} \mathrm{H}\right\} \mathrm{NMR}\left(\mathrm{CDCl}_{3}, 101 \mathrm{MHz}\right)$

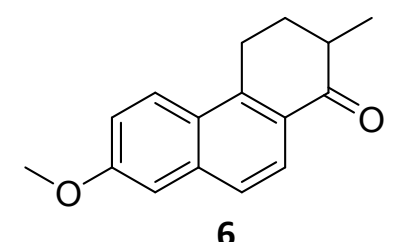

6

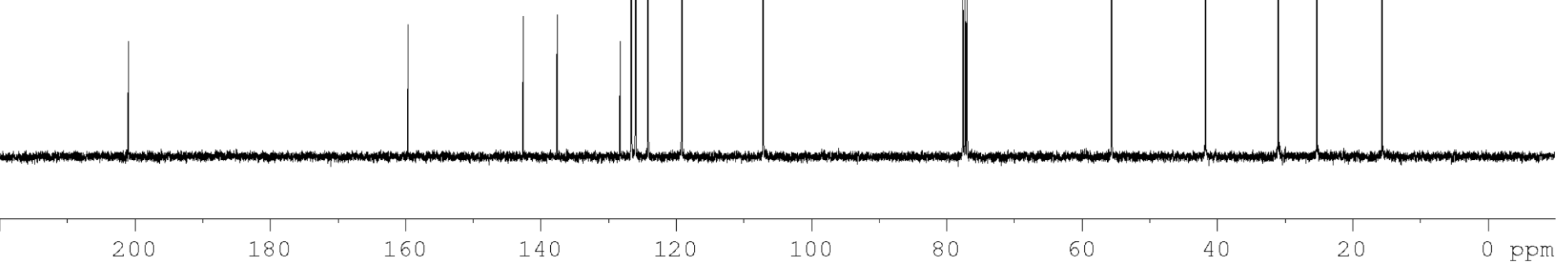

$\mathrm{CDCl}_{3}$ 


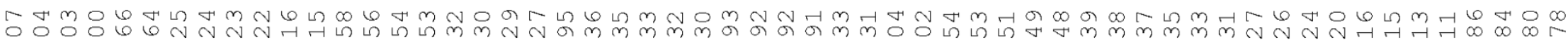

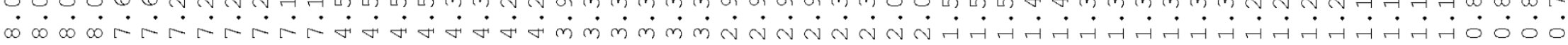

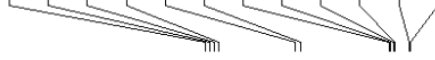

${ }^{1} \mathrm{H} \mathrm{NMR}\left(\mathrm{CDCl}_{3}, 400 \mathrm{MHz}\right)$

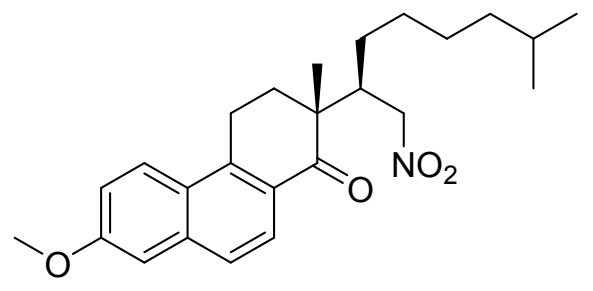

(士)-7, 4:1 d.r.

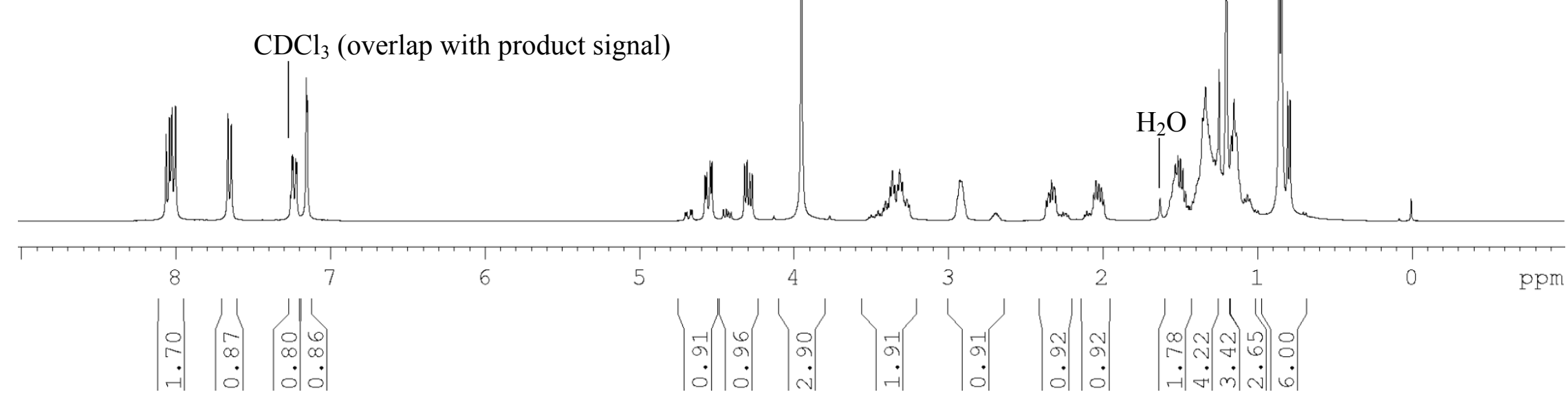



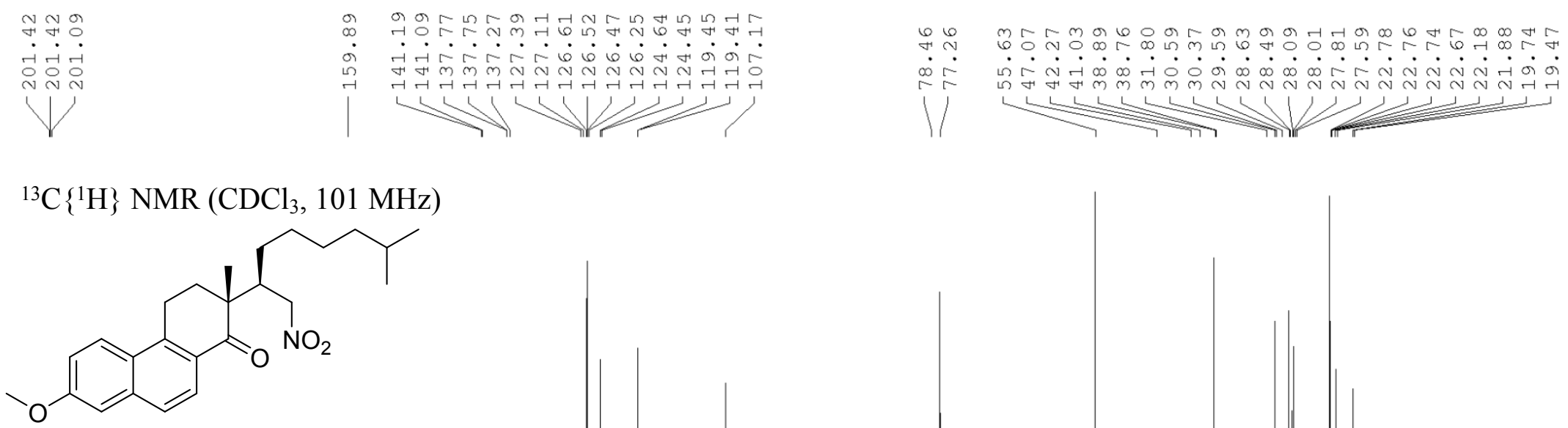

( \pm )-7, 4:1 d.r.

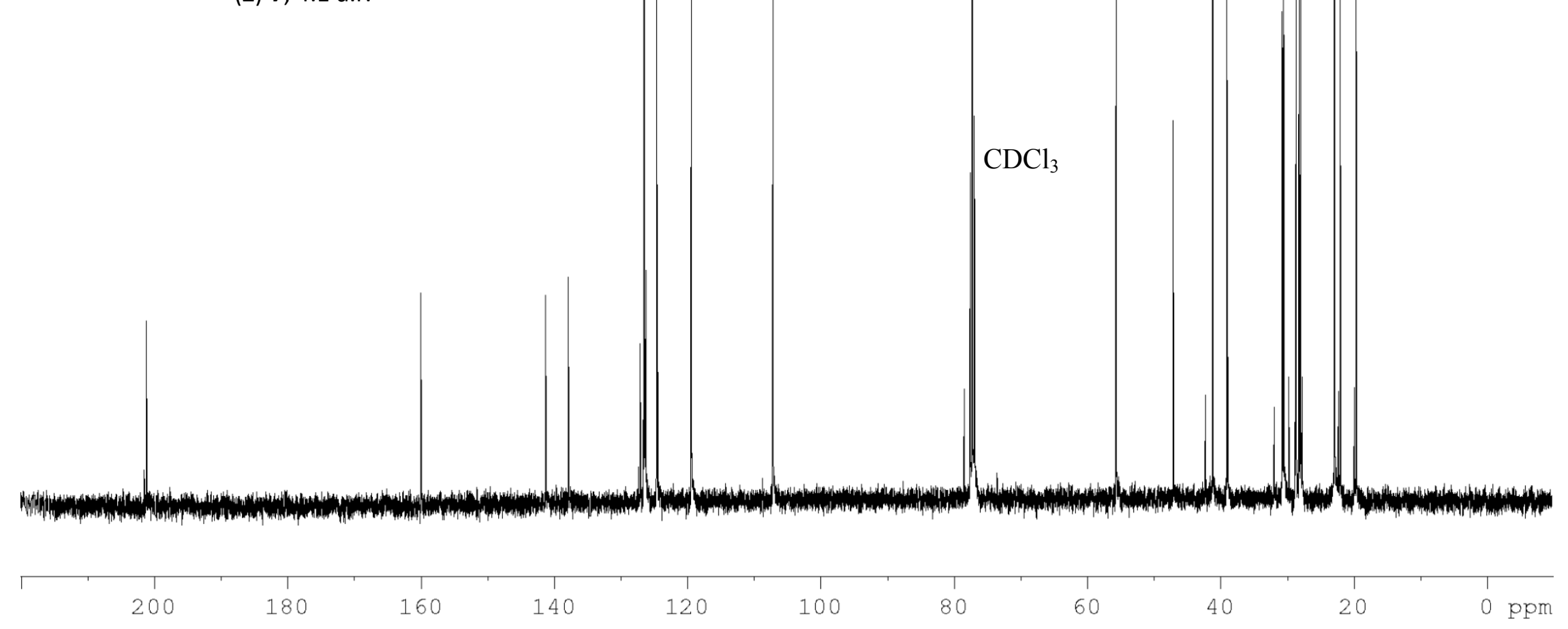



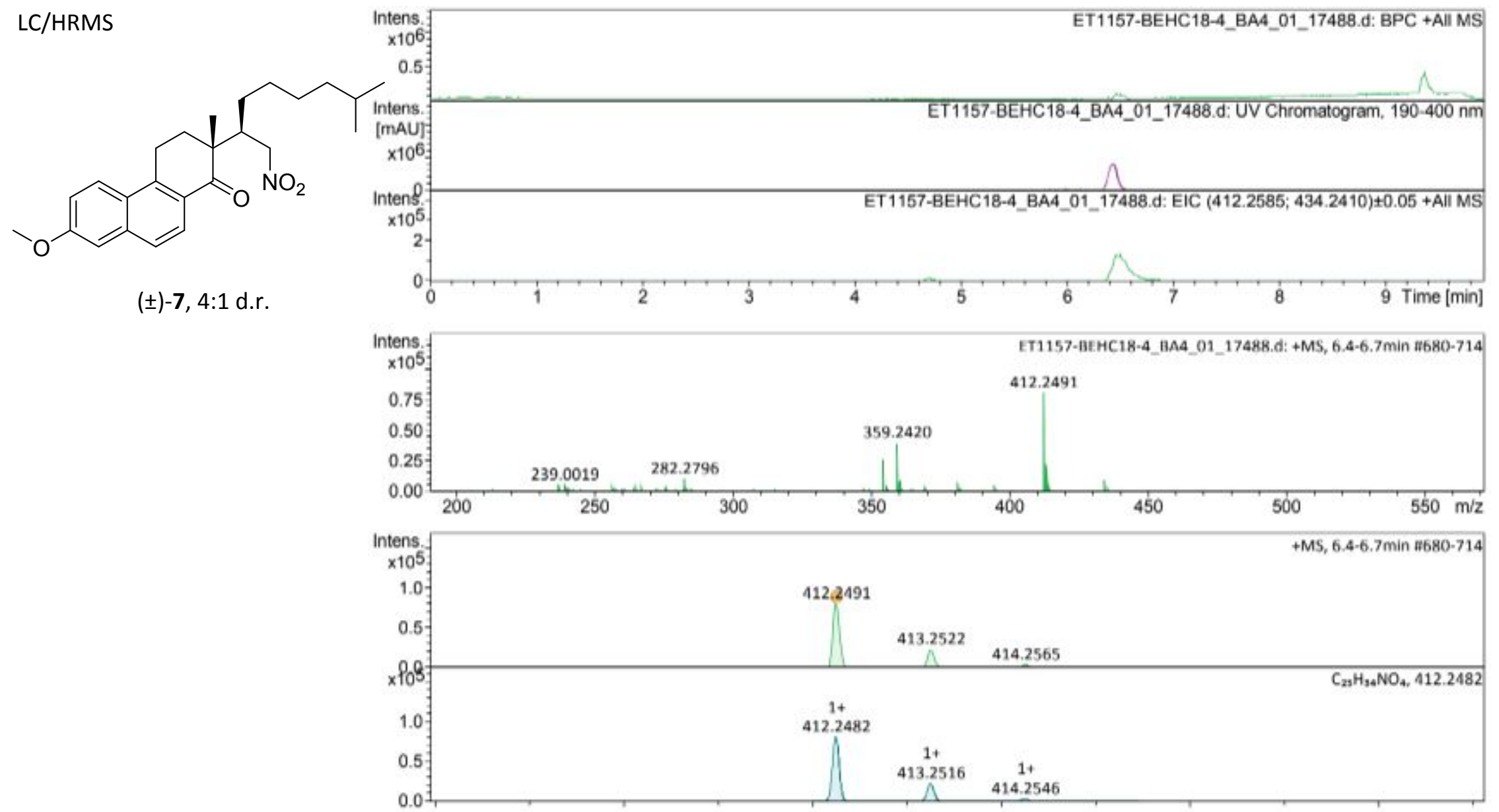

$\begin{array}{rrlrrrrrrrr}\text { Meas. } \mathrm{m} / \mathrm{z} & \text { \# } & \text { lon Formula } & \mathrm{m} / \mathrm{z} & \text { err [ppm] } & \text { mSigma } & \text { \# mSigma } & \text { Score } & \text { rdb } & \mathrm{e}^{-} \text {Conf } & \mathrm{N}-\mathrm{Rule} \\ 412.2491 & 1 & \mathrm{C} 25 \mathrm{H} 34 \mathrm{NO4} & 412.2482 & -2.2 & 1.5 & 1 & 100.00 & 9.5 & \text { even } & \text { ok } \\ & 2 & \text { C28H32N2O } & 412.2509 & 4.3 & 21.5 & 2 & 39.82 & 14.0 & \text { odd } & \text { ok }\end{array}$


FTIR (ATR)

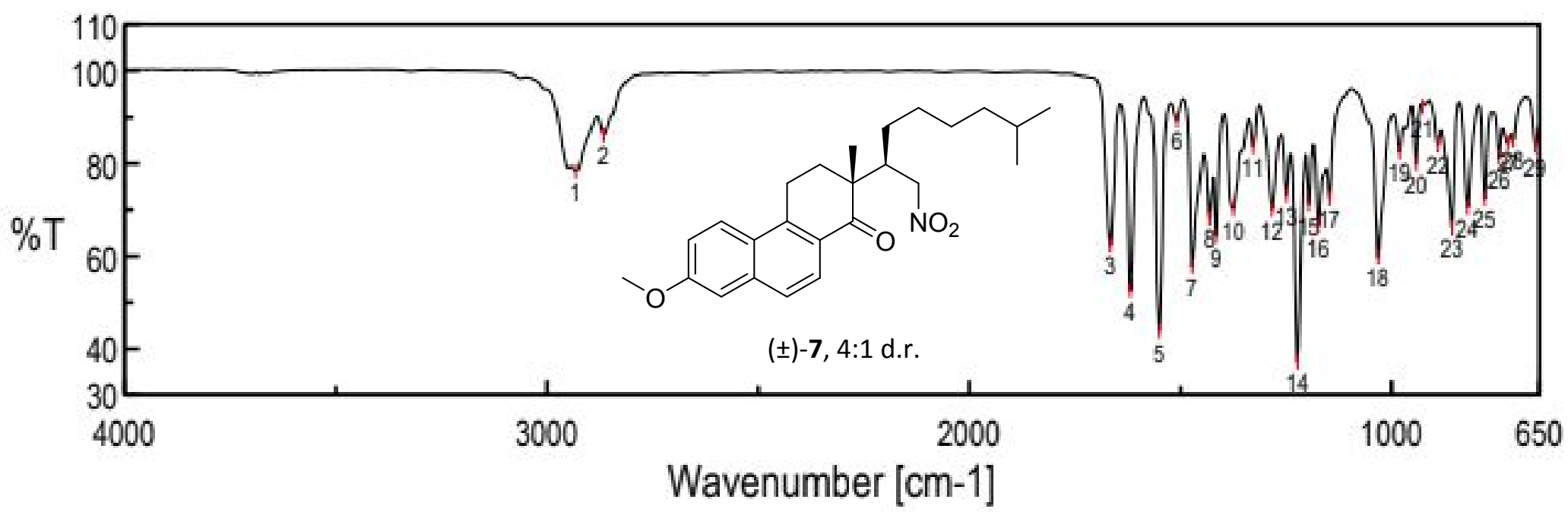

[ Result of Peak Picking ]

No. Position Intensity

$\begin{array}{lll}1 & 2932.23 & 78.2624\end{array}$

$4 \quad 1618.95 \quad 52.4881$

$\begin{array}{lll}7 & 1472.38 & 57.6392\end{array}$

$\begin{array}{lll}10 & 1375 & 70.1228\end{array}$

$13 \quad 1248.68 \quad 73.0198$

$16 \quad 1173.47 \quad 66.3863$

$\begin{array}{lll}19 & 980.625 & 82.3789\end{array}$

$\begin{array}{lll}22 & 890.952 \quad 83.9394\end{array}$

$\begin{array}{lll}25 & 780.065 & 72.3196\end{array}$

$\begin{array}{lll}28 & 711.604 & 84.9922\end{array}$

$\begin{array}{lll}\text { No. } & \text { Position } & \text { Intensity } \\ 2 & 2865.7 & 86.1378 \\ 5 & 1550.49 & 43.6922 \\ 8 & 1430.92 & 68.1391 \\ 11 & 1327.75 & 83.5725 \\ 14 & 1222.65 & 37.0511 \\ 17 & 1147.44 & 72.3762 \\ 20 & 942.056 & 79.8766 \\ 23 & 857.204 & 66.091 \\ 26 & 745.352 & 81.2227 \\ 29 & 659.536 & 83.8596\end{array}$

$\begin{array}{lll}\text { No. } & \text { Position } & \text { Intensity } \\ 3 & 1666.2 & 62.3549 \\ 6 & 1508.06 & 89.2697 \\ 9 & 1415.49 & 64.2543 \\ 12 & 1283.39 & 70.003 \\ 15 & 1195.65 & 71.0935 \\ 18 & 1032.69 & 59.6957 \\ 21 & 926.628 & 92.223 \\ 24 & 820.563 & 70.4549 \\ 27 & 725.104 & 84.4279\end{array}$




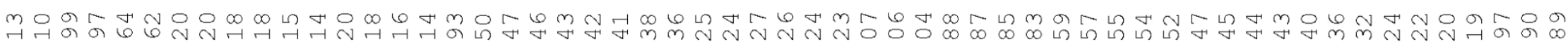

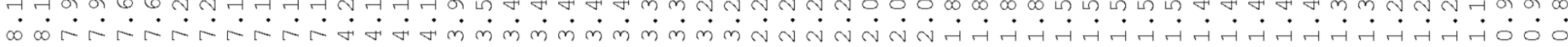

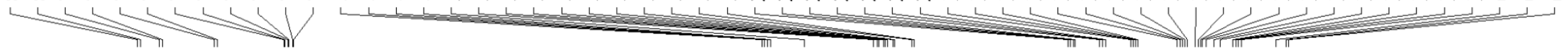

${ }^{1} \mathrm{H} \mathrm{NMR}\left(\mathrm{CDCl}_{3}, 400 \mathrm{MHz}\right)$

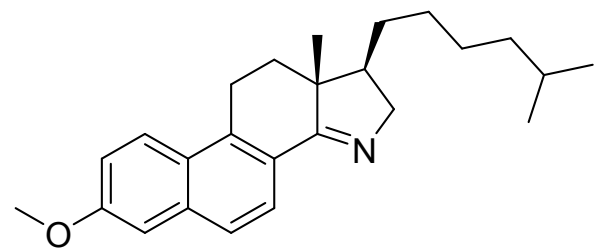

$( \pm)-8$

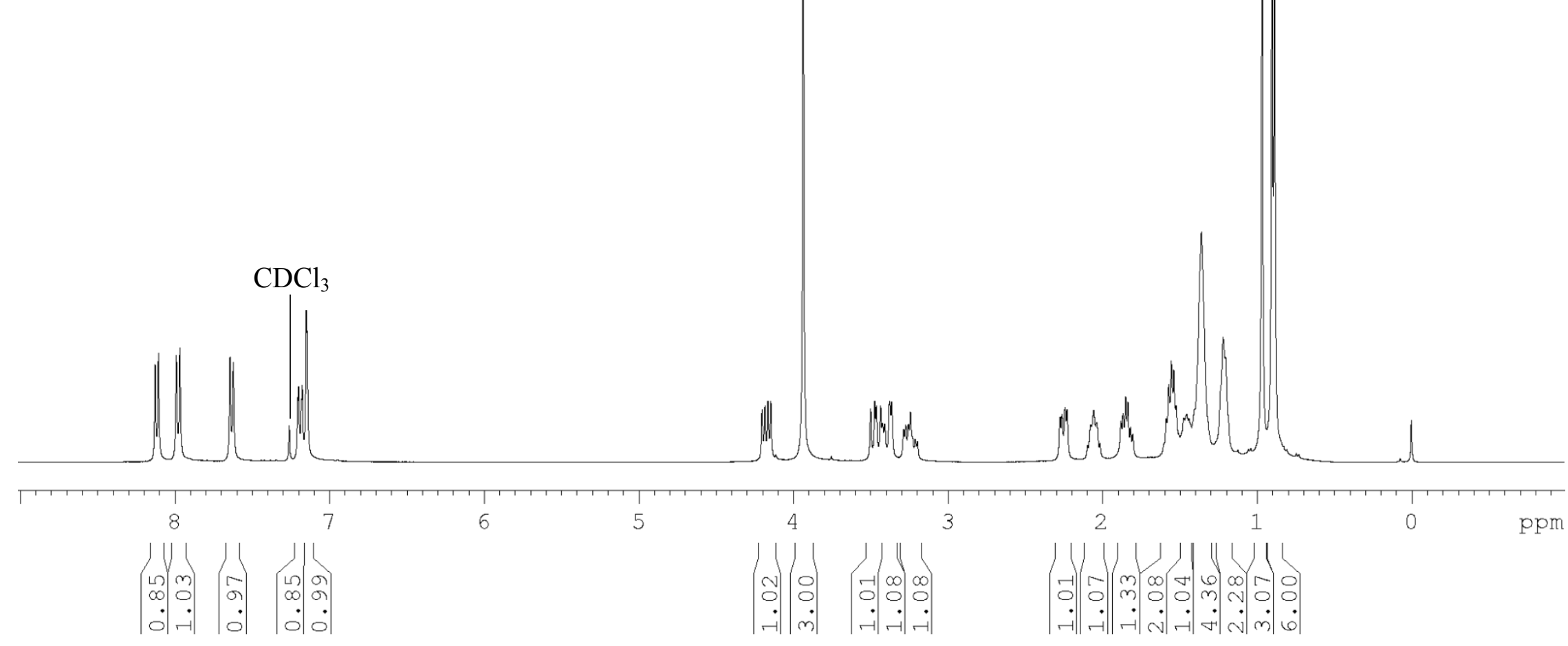




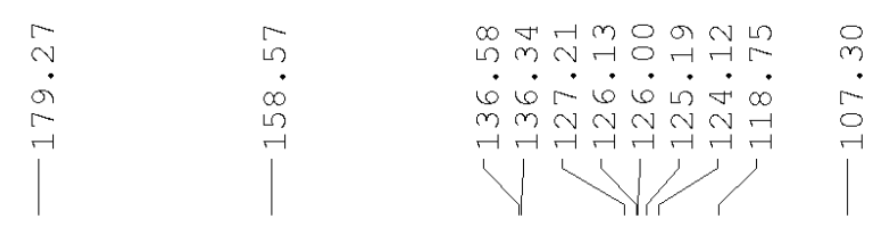

${ }^{13} \mathrm{C}\left\{{ }^{1} \mathrm{H}\right\} \mathrm{NMR}\left(\mathrm{CDCl}_{3}, 201 \mathrm{MHz}\right)$

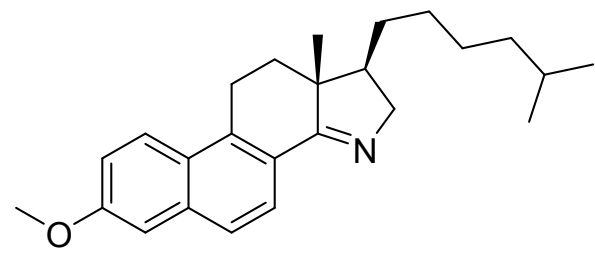

$( \pm)-8$

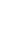

(1)

$$
\text { 웅 }
$$

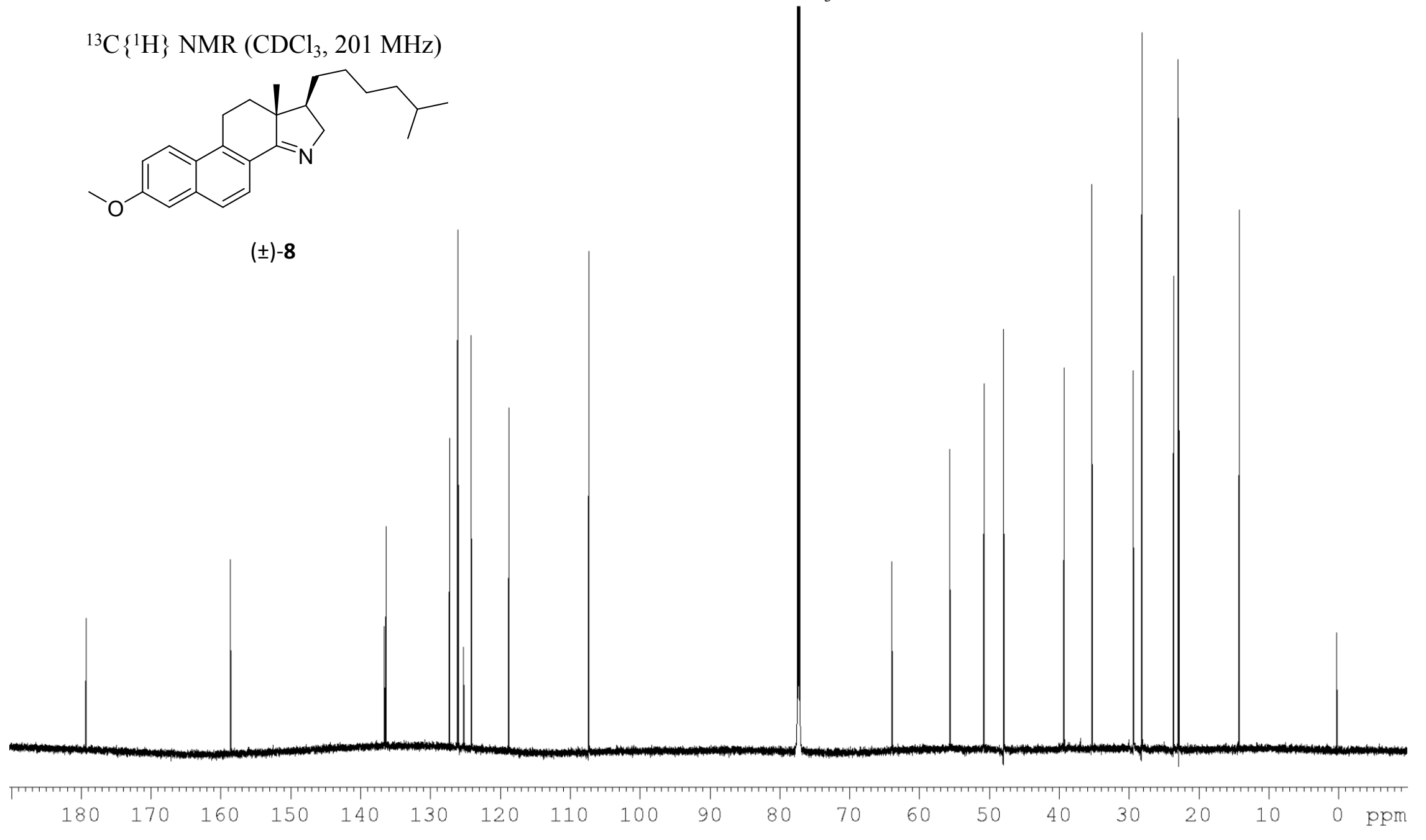

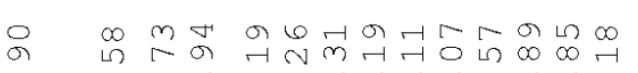

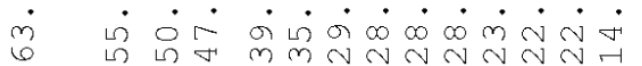

$\mathrm{CDCl}_{3}$

\section{(1)}

11 Nit)

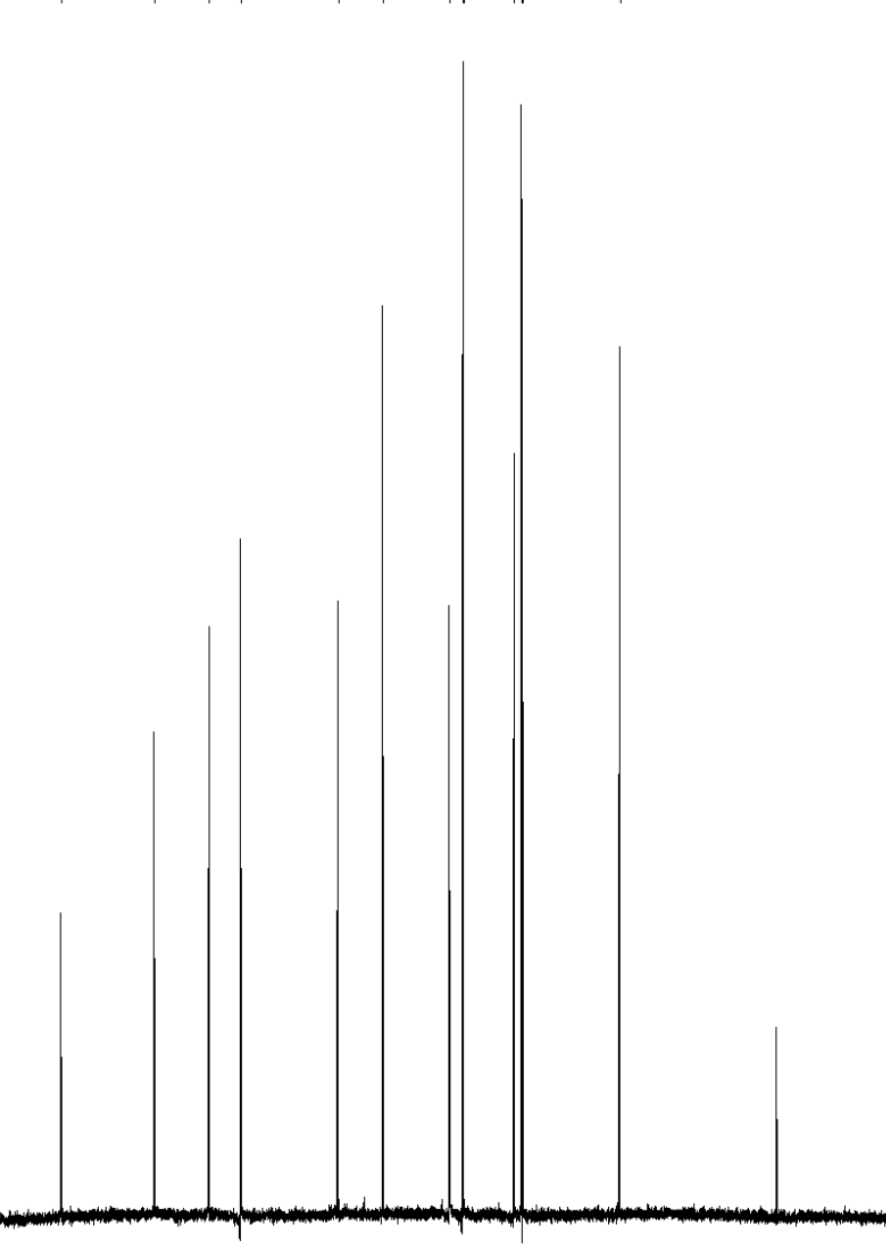

$\mathrm{pm}$ 


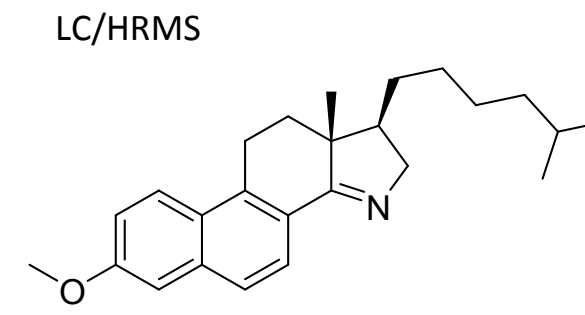

(士)-8
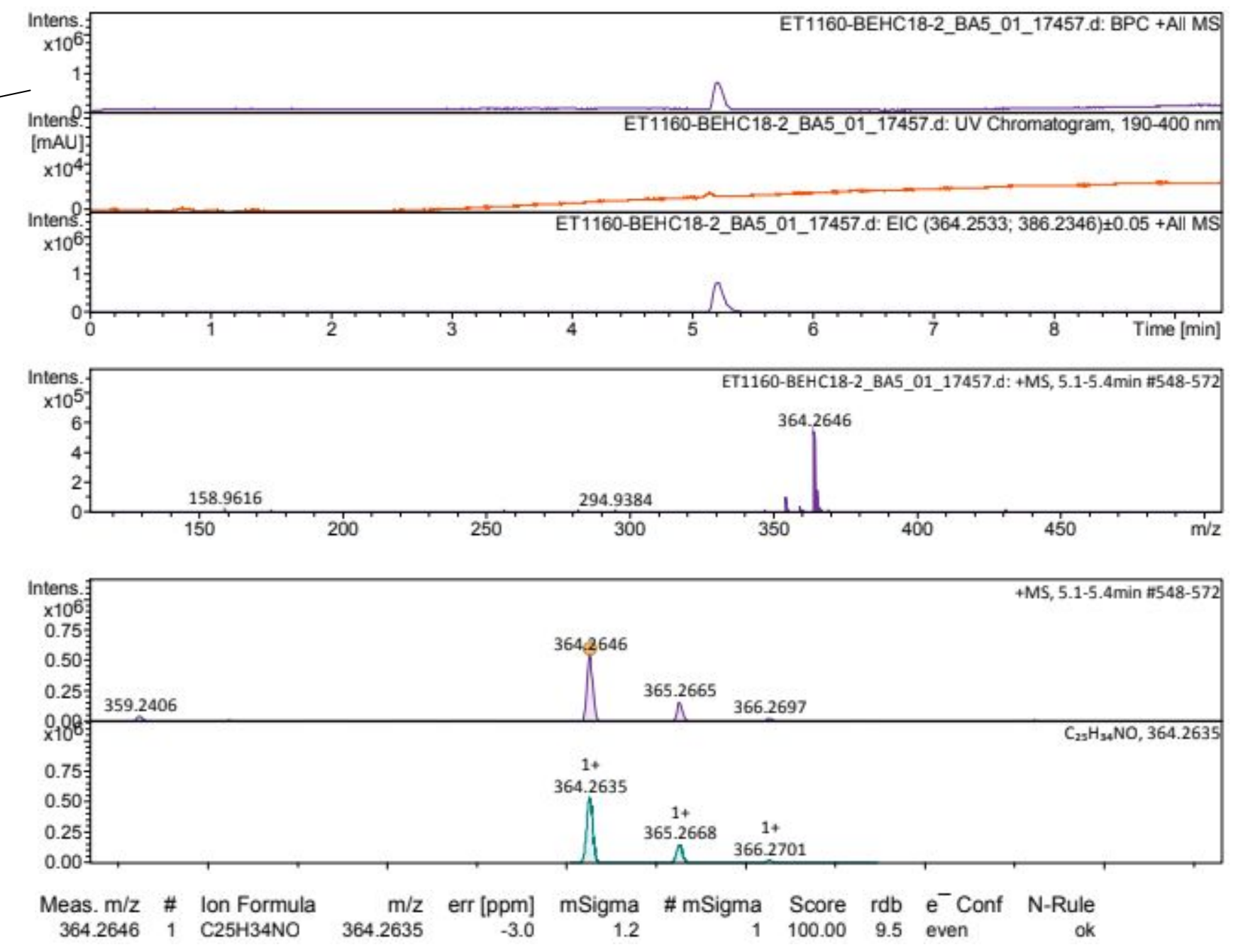
FTIR (ATR)

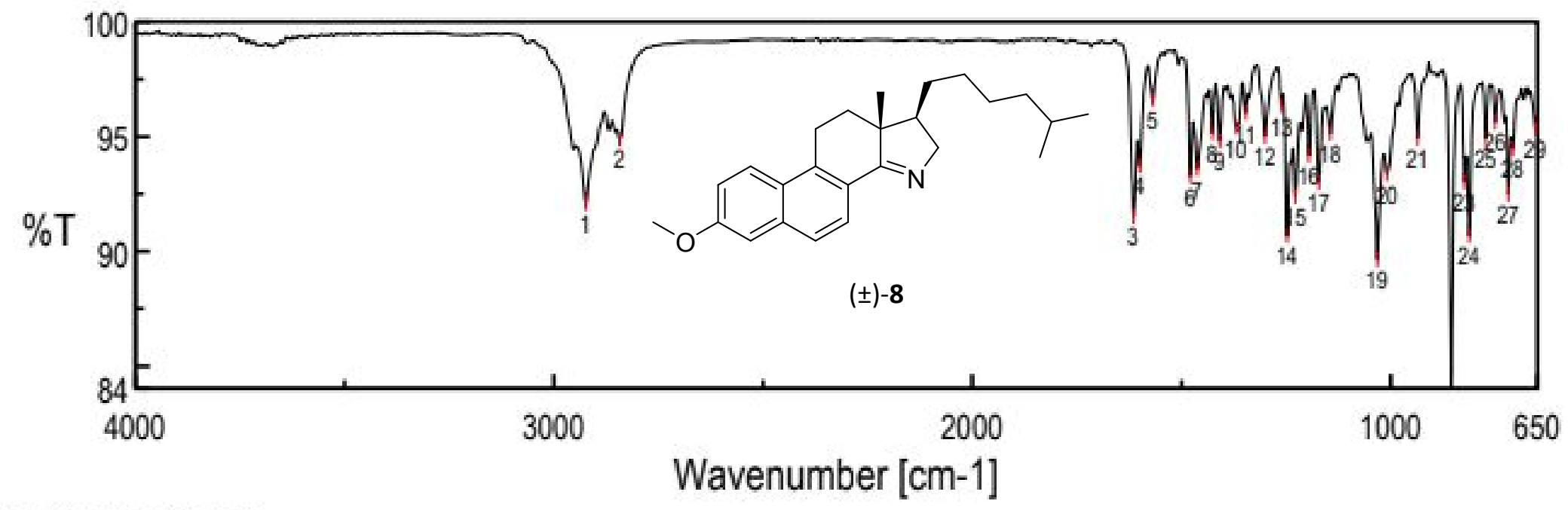

[ Result of Peak Picking]

$\begin{array}{lllllllll}\text { No. } & \text { Position } & \text { Intensity } & \text { No. } & \text { Position } & \text { Intensity } & \text { No. Position } & \text { Intensity } \\ 1 & 2923.56 & 92.1016 & 2 & 2842.56 & 94.888 & 3 & 1615.09 & 91.5241 \\ 4 & 1599.66 & 93.7203 & 5 & 1568.81 & 96.6273 & 6 & 1479.13 & 93.2652 \\ 7 & 1462.74 & 93.5973 & 8 & 1427.07 & 95.1589 & 9 & 1408.75 & 94.812 \\ 10 & 1370.18 & 95.3893 & 11 & 1347.03 & 96.0281 & 12 & 1300.75 & 95.0127 \\ 13 & 1261.22 & 96.3403 & 14 & 1247.72 & 90.6604 & 15 & 1228.43 & 92.3745 \\ 16 & 1195.65 & 94.1846 & 17 & 1171.54 & 92.997 & 18 & 1144.55 & 95.1278 \\ 19 & 1032.69 & 89.6364 & 20 & 1008.59 & 93.3701 & 21 & 936.271 & 94.9282 \\ 22 & 855.275 & 84.0031 & 23 & 824.42 & 93.0169 & 24 & 812.849 & 90.6626 \\ 25 & 773.315 & 94.9111 & 26 & 751.138 & 95.6288 & 27 & 719.318 & 92.4838 \\ 28 & 708.712 & 94.4662 & 29 & 654.715 & 95.3322 & & & \end{array}$


${ }^{1} \mathrm{H} \mathrm{NMR}\left(\mathrm{CDCl}_{3}, 800 \mathrm{MHz}\right)$

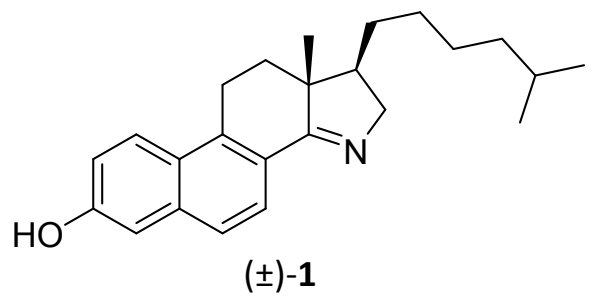

$( \pm)-1$
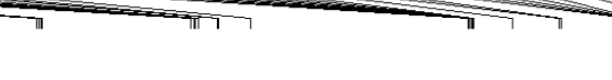

III

eOH (overlap with product signal)
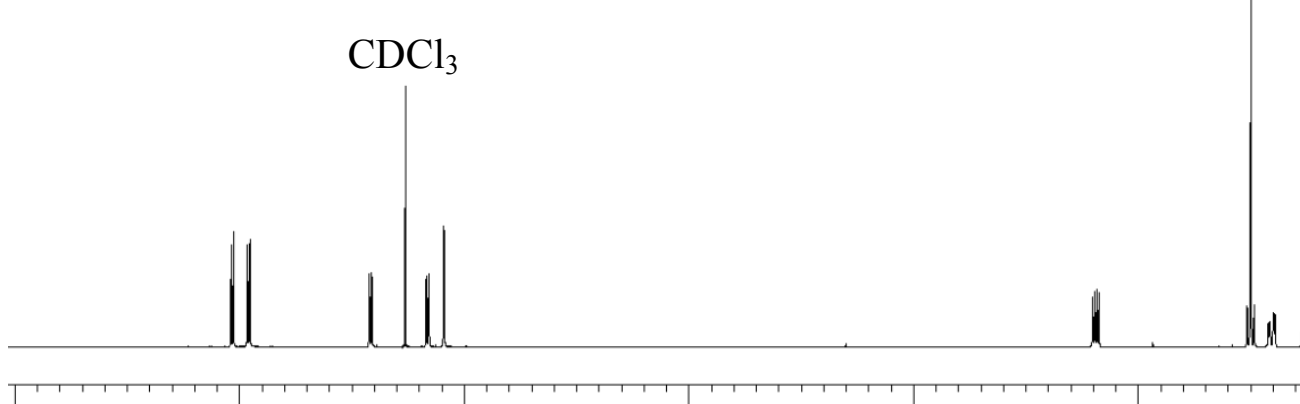

7

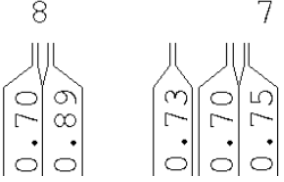

6

5

1, 1, 1, 1, 1, 1, 1, 1

(1) 


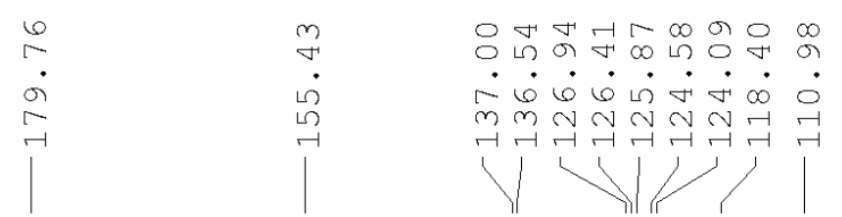

${ }^{13} \mathrm{C}\left\{{ }^{1} \mathrm{H}\right\} \mathrm{NMR}\left(\mathrm{CDCl}_{3}, 201 \mathrm{MHz}\right)$

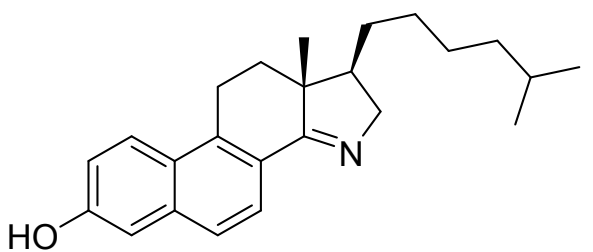

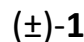

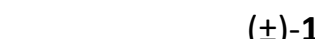

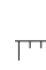

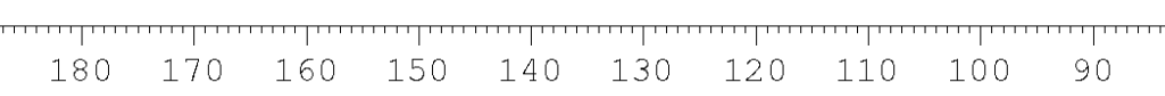

$\begin{array}{lllll}180 & 150 & 140 & 130\end{array}$
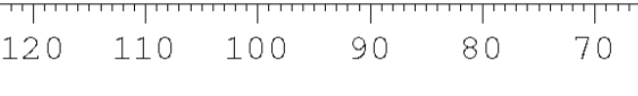

60
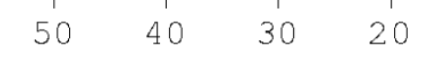

10

0 ppm 

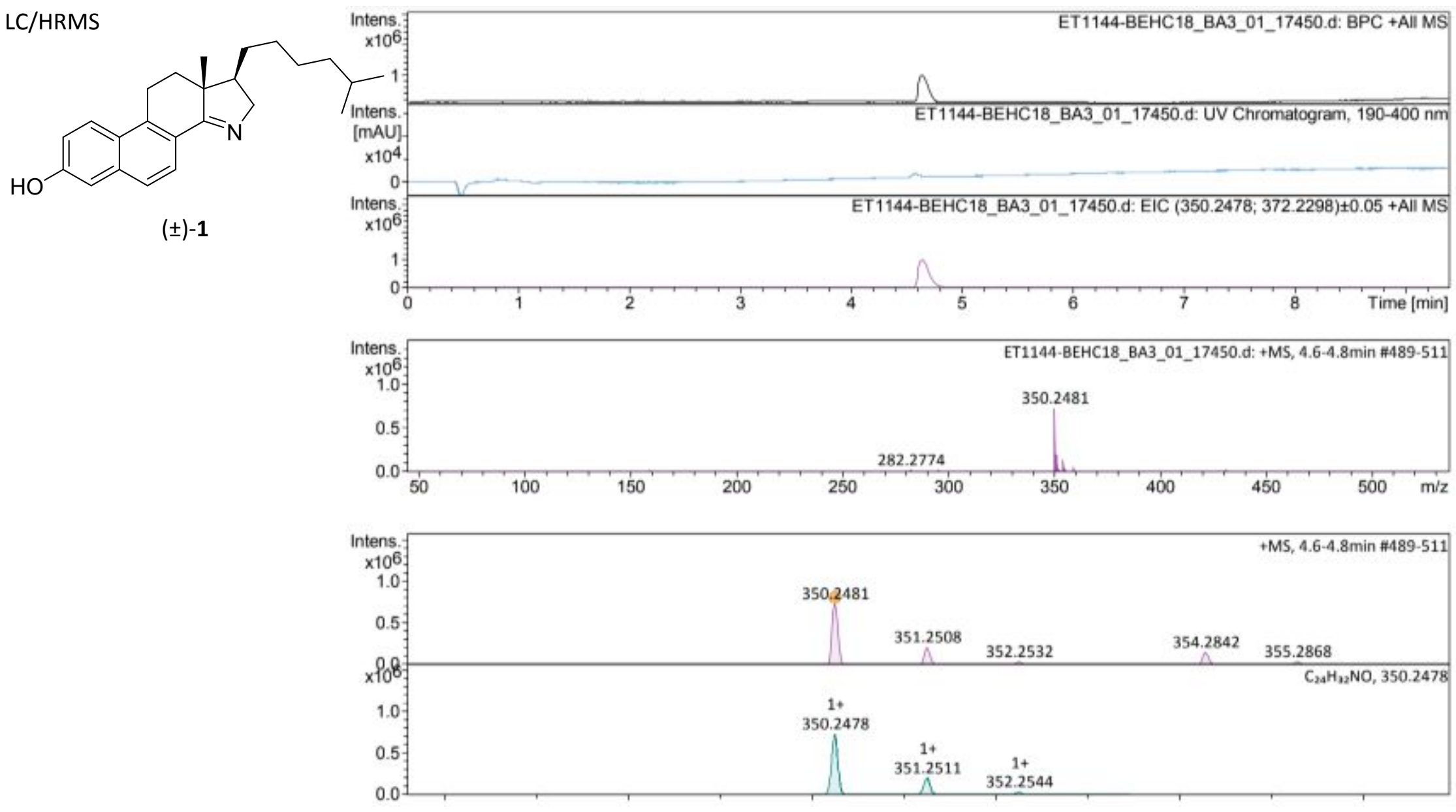

Meas. $\mathrm{m} / \mathrm{z}$ \# lon Formula $\mathrm{m} / \mathrm{z}$ err [ppm] $\quad$ mSigma \# mSigma Score $\mathrm{rdb} \mathrm{e}^{-}$Conf $\quad \mathrm{N}$-Rule

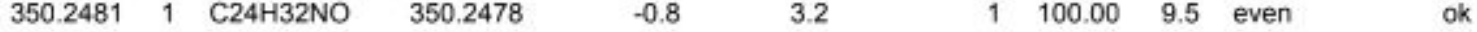


FTIR (ATR)

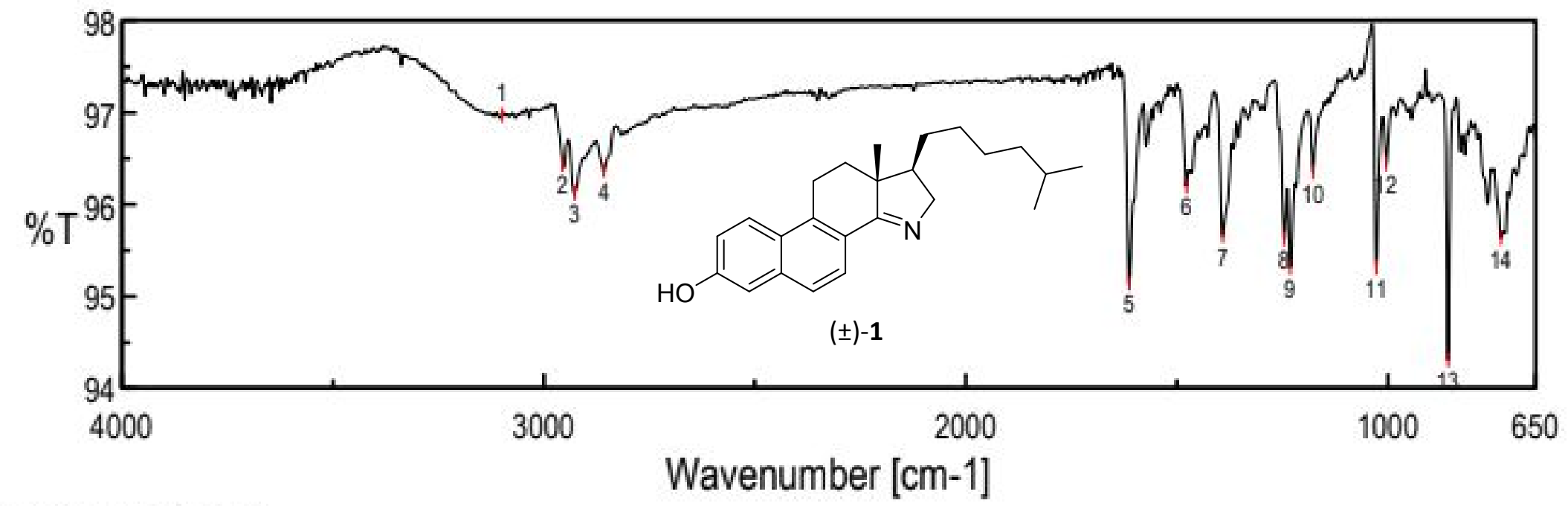

[ Result of Peak Picking ]

No. Position Intensity

13100.01

No. Position

100.0

96.9739

$1177.33 \quad 96.3502$

22955.38

Intensity

No. Position

intensity

$7 \quad 1391.39$

$13 \quad 857.204 \quad 94.3073$

$5 \quad 1613.16$

96.4305

95.1387

95.6246

95.3136

$3 \quad 2927.41$

96.1278

$8 \quad 1245.79$

$11 \quad 1027.87$

95.6302

$\begin{array}{lll}6 & 1477.21 & 96.1929\end{array}$

$\begin{array}{lll}9 & 1231.33 & 95.3155\end{array}$

$\begin{array}{lll}12 & 1003.77 & 96.4413\end{array}$ 


\section{References}

(1) Melhuish, W. H. Quantum Efficiencies of Fluorescence of Organic Substances: Effect of Solvent and Concentration of the Fluorescent Solute. J. Phys. Chem. 1961, 65, 229-235.

(2) Frisch, M. J.; Trucks, G. W.; Schlegel, H. B.; Scuseria, G. E.; Robb, M. A.; Cheeseman, J. R.; Scalmani, G.; Barone, V.; Petersson, G. A.; Nakatsuji, H.; Li, X.; Caricato, M.; Marenich, A. V.; Bloino, J.; Janesko, B. G.; Gomperts, R.; Mennucci, B.; Hratchian, H. P.; Ortiz, J. V.; Izmaylov, A. F.; Sonnenberg, J. L.; Williams-Young, D.; Ding, F.; Lipparini, F.; Egidi, F.; Goings, J.; Peng, B.; Petrone, A.; Henderson, T.; Ranasinghe, D.; Zakrzewski, V. G.; Gao, J.; Rega, N.; Zheng, G.Liang, W.; Hada, M.; Ehara, M.; Toyota, K.; Fukuda, R.; Hasegawa, J.; Ishida, M.; Nakajima, T.; Honda, Y.; Kitao, O.; Nakai, H.; Vreven, T.; Throssell, K.; Montgomery, J. A., Jr.; Peralta, J. E.; Ogliaro, F.; Bearpark, M. J.; Heyd, J. J.; Brothers, E. N.; Kudin, K. N.; Staroverov, V. N.; Keith, T. A.; Kobayashi, R.; Normand, J.; Raghavachari, K.; Rendell, A. P.; Burant, J. C.; Iyengar, S. S.; Tomasi, J.; Cossi, M.; Millam, J. M.; Klene, M.; Adamo, C.; Cammi, R.; Ochterski, J. W.; Martin, R. L.; Morokuma, K.; Farkas, O.; Foresman, J. B.; Fox, D. J. Gaussian 16, Revision B.01. Gaussian, Inc.: Wallingford, CT 2016.

(3) Adamo, C.; Jacquemin, D. The Calculations of Excited-State Properties with TimeDependent Density Functional Theory. Chem. Soc. Rev. 2013, 42, 845-856.

(4) Laurent, A. D.; Adamo, C.; Jacquemin, D. Dye Chemistry with Time-Dependent Density Functional Theory. Phys. Chem. Chem. Phys. 2014, 16, 14334-14356.

(5) Sears, J. S.; Koerzdoerfer, T.; Zhang, C. R.; Brédas, J. L. Orbital Instabilities and Triplet States from Time-Dependent Density Functional Theory and Long-Range Corrected Functionals. J. Chem. Phys. 2011, 135, 151103.

(6) Modzel, M.; Lund, F. W.; Wüstner, D. Synthesis and Live-Cell Imaging of Fluorescent Sterols for Analysis of Intracellular Cholesterol Transport. In Cholesterol Homeostasis; Gelissen, I., Brown, A., Eds.; Humana Press: New York, 2017; pp 111-140.

Wüstner, D.; Larsen, A. L.; Færgeman, N. J.; Brewer, J. R.; Sage, D. Selective Visualization of Fluorescent Sterols in Caenorhabditis Elegans by Bleach-Rate Based Image Segmentation. Traffic 2010, 11, 440-454.

(8) Wüstner, D.; Brewer, J. R.; Bagatolli, L. A.; Sage, D. Potential of Ultraviolet Widefield Imaging and Multiphoton Microscopy for Analysis of Dehydroergosterol in Cellular Membranes. Microsc. Res. Tech. 2011, 74, 92-108.

(9) Small-Molecule Drug Discovery Suite. Schrödinger, LLC: New York 2019.

(10) Friesner, R. A.; Banks, J. L.; Murphy, R. B.; Halgren, T. A.; Klicic, J. J.; Mainz, D. T.; Repasky, M. P.; Knoll, E. H.; Shelley, M.; Perry, J. K.; Shaw, D. E.; Francis, P.; Shenkin, P. S. Glide: A New Approach for Rapid, Accurate Docking and Scoring. 1. Method and Assessment of Docking Accuracy. J. Med. Chem 2004, 47, 1739-1749.

(11) Halgren, T. A.; Murphy, R. B.; Friesner, R. A.; Beard, H. S.; Frye, L. L.; Pollard, W. T.; Banks, J. L. Glide: A New Approach for Rapid, Accurate Docking and Scoring. 2. Enrichment Factors in Database Screening. J. Med. Chem. 2004, 47, 1750-1759. Friesner, R. A.; Murphy, R. B.; Repasky, M. P.; Frye, L. L.; Greenwood, J. R.; Halgren, T. A.; Sanschagrin, P. C.; Mainz, D. T. Extra Precision Glide: Docking and Scoring Incorporating a Model of Hydrophobic Enclosure for Protein-Ligand Complexes. J. Med. Chem. 2006, 49, 6177-6196.

(13) Case, D. A.; Ben-Shalom, I. Y.; Brozell, S. R.; Cerutti, D. S.; Cheatham, III, T. E.; Cruzeiro, V. W. D.; Darden, T. A.; Duke, R. E.; Ghoreishi, D.; Gilson, M. K.; Gohlke, D.; 
Goetz, A. W.; Greene, D.; Harris, R.; Homeyer, N.; Izadi, S.; Kovalenko, A. Kurtzman, T.; Lee, T. S.; LeGrand, S.; Li, P.; Lin, C.; Liu, J.; Luchko, T.; Luo, R.; Mermelstein, D. J.; Merz, K. M.; Miao, Y.; Monard, G.; Nguyen, C.; Nguyen, H.; Omelyan, I.; Onufriev, A.; Pan, F.; Qi, R.; Roe, D. R. Roitberg, A.; Sagui, C.; Schott-Verdugo, S.; Shen, J.; Simmerling, C. L.; Smith, J.; Salomon-Ferrer, R.; Swails, J.; Walker, R. C.; Wang, J.; Wei, H.; Wolf, R. M.; Wu, X.; Xiao, L.; York, D. M.; Kollman, P. A. AMBER 2018. University of California: San Francisco 2018.

(14) Ponder, J. W.; Case, D. A. Force Fields for Protein Simulations. Adv. Prot. Chem. 2003, $66,27-85$.

(15) Maier, J. A.; Martinez, C.; Kasavajhala, K.; Wickstrom, L.; Hauser, K. E.; Simmerling, C. Ff14SB: Improving the Accuracy of Protein Side Chain and Backbone Parameters from Ff99SB. J. Chem. Theory Comput. 2015, 11, 3696-3713. 